\title{
Molecular Communications in Complex Systems of Dynamic Supramolecular Polymers
}

\author{
Martina Crippa ${ }^{1}$, Claudio Perego ${ }^{2}$, Anna L. de Marco ${ }^{2,3}$, and Giovanni M. Pavan ${ }^{1,2, *}$ \\ ${ }^{1}$ Department of Applied Science and Technology, Politecnico di Torino, Corso Duca degli Abruzzi 24, 10129 Torino, \\ Italy \\ ${ }^{2}$ Department of Innovative Technologies, University of Applied Sciences and Arts of Southern Switzerland, Polo \\ Universitario Lugano, Campus Est, Via la Santa 1, 6962 Lugano-Viganello, Switzerland \\ ${ }^{3}$ Department of Physics, Università degli Studi di Genova, Via Dodecaneso 33, 16100 Genova, Italy \\ *corresponding author: Giovanni M. Pavan (giovanni.pavan@polito.it)
}

ABSTRACT

Supramolecular polymers are composed of monomers that self-assemble non-covalently, generating distributions of monodimensional fibres in continuous communication with each other and with the surrounding solution. Fibres, exchanging molecular species, and external environment constitute a sole complex system, which intrinsic dynamics is hard to elucidate. Here we report coarse-grained molecular simulations that allow studying supramolecular polymers at the thermodynamic equilibrium, explicitly showing the complex nature of these systems, which are composed of exquisitely dynamic molecular entities. Detailed studies of molecular exchange provide insights into key factors controlling how assemblies communicate with each other, defining the equilibrium dynamics of the system. Using minimalistic and finer chemically relevant molecular models, we observe that a rich concerted complexity is intrinsic in such self-assembling systems. This offers a new dynamic and probabilistic (rather than structural) picture of supramolecular polymer systems, where the travelling molecular species continuously shape the assemblies that statistically emerge at the equilibrium.

\section{Introduction}

Synthetic supramolecular polymers, composed of monomers that self-assemble in solution via reversible, non-covalent interactions, are important platforms for the development of advanced materials for many applications. ${ }^{1-4}$ Such polymers are formed by monomeric units interacting via hydrogen bonding, solvophobic repulsion, $\pi$ - $\pi$ stacking, etc. ${ }^{1,5}$ Thanks to such reversible interactions, supramolecular polymers bear unique dynamical properties at the equilibrium, which are at the basis of stimuli-responsive and adaptive features reminiscent of living materials. ${ }^{6-9}$

While, in principle, changing the monomer structure allows tuning the dynamic properties of supramolecular polymers, the complexity of these systems makes it often impossible to obtain clear monomer-assembly relationships. Various experimental approaches were used to characterize the structure of supramolecular polymers in solution, ${ }^{5,10-14}$ most often providing average information on the entire solution, with limited detail on the distribution of aggregate sizes. Considerable efforts were spent also in characterizing the dynamics of supramolecular polymers using, e.g., Förster Resonance Energy Transfer (FRET), ${ }^{15,16}$ Stochastic Optical Reconstruction Microscopy (STORM),${ }^{16-18}$ or Hydrogen/deuterium exchange (HDX) mass spectrometry. ${ }^{19,20}$ Typically, such approaches allow studying supramolecular polymer dynamics at the level of statistical ensembles or of individual assemblies (e.g., using bulky labeling groups, STORM detects supramolecular exchange with a resolution of $\sim 20-50 \mathrm{~nm}){ }^{16-18,21,22}$ However, obtaining a clear understanding of the molecular processes that, in concert, define the dynamic nature of supramolecular polymers would ideally require a complete characterization of such systems with submolecular resolution, which is mostly inaccessible via experiments.

Computational modelling recently emerged as a crucial tool to investigate the dynamic nature of supramolecular polymers. Stochastic polymerisation models ${ }^{23}$ have been employed to study the self-assembly of amyloid fibres ${ }^{24}$ or synthetic selfassembling systems, such as e.g. 1,3,5-benzenetricarboxamides (BTAs) ${ }^{25}$ or phenylenevinylenes. ${ }^{26}$ Such models can predict the evolution of the aggregate population, resulting in polymerisation curves that are validated by fitting the signals detected in experiments based on hypotetical polymerization schemes. ${ }^{27-29}$ At atomistic resolution, first-principles molecular dynamics (MD) simulations were recently demonstrated useful for the study of supramolecular polymers. ${ }^{16,30-34}$ Coarse-grained MD (CG-MD) simulations have been also employed to study complex supramolecular polymer systems, reaching relevant space and time-scales for the study of their dynamics. ${ }^{35-43}$ In particular, combined with enhanced sampling methods, such models allow to study the molecular exchange between the supramolecular structures and their surroundings, ${ }^{21,31,44-47}$ the exchange pathways, ${ }^{48}$ and the response of these materials to external stimuli. ${ }^{49,50}$ Such advanced computational approaches 
a

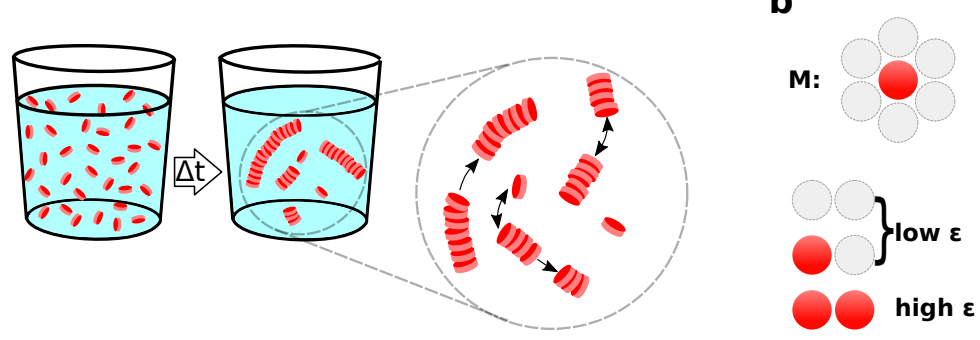

C

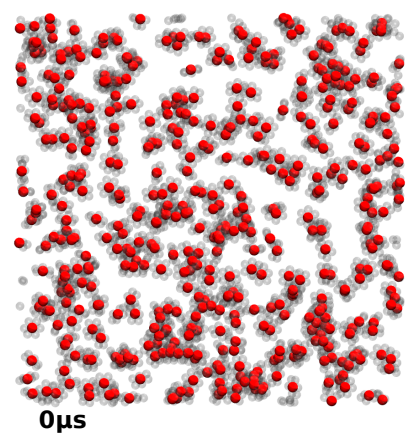

b

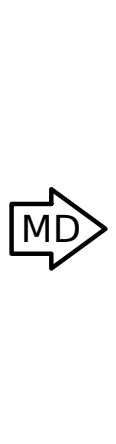

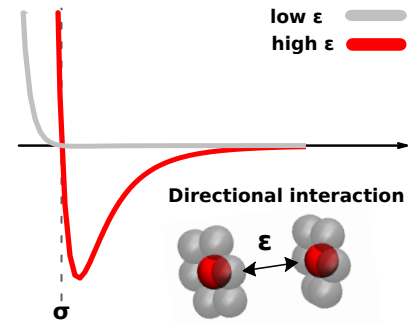

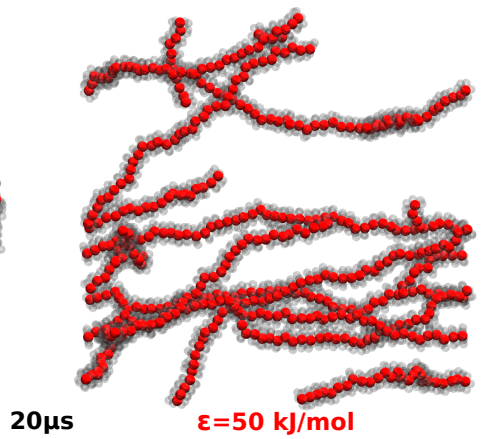

Figure 1. Minimalistic model of self-assembling monomers M. (a) Scheme for the self-assembly of monomeric $\mathbf{M}$ units into supramolecular polymers. At the thermodynamic equilibrium, the fibres exhibit a more/less pronounced dynamic behavior, exchanging units and fragments with with each other and with the external environment. (b) Structure and interaction of the minimalistic model: the $\mathbf{M}$ monomers interact directionally via attractive interaction between the central red beads. Weakly interacting beads (in gray) are added to screen the red beads and prevent lateral binding of the monomers (imparting directionality to the $\mathbf{M}-\mathbf{M}$ interaction). The interactions in the model are defined by Lennard-Jones potentials. (c) CG-MD simulation snapshots of a model system composed of 500 initially randomly distributed monomers: the two snapshots at $t_{C G}=20 \mu \mathrm{s}$ refer to cases with the interaction strength between central beads is set at $\varepsilon=40 \mathrm{~kJ} \mathrm{~mol}^{-1}$ (center) or $\varepsilon=50 \mathrm{~kJ} \mathrm{~mol}^{-1}$ (right) respectively (both model systems start from the same initial condition, on the left).

are mostly employed to study isolated assemblies in solution. But the dynamics of a system involving multiple assemblies (e.g., monomers/oligomers exchange between fibres, or fibres fragmentation and recombination) remains typically overlooked. To understand how supramolecular polymers communicate with each other in a realistic system, it is necessary to simulate multiple assemblies in dynamic, supramolecular equilibrium conditions, studying their complex, concerted behavior with sufficiently high resolution.

Inspired by simulation studies in the field of colloids, ${ }^{51-53}$ we here designed an inverse multiscale modeling approach, allowing us to study supramolecular polymers as complex molecular systems. Minimalistic CG models allow to reconstruct the network of molecular exchange events between the assemblies in these systems at the thermodynamic equilibrium. We can observe how these complex systems are composed of dynamic entities in continuous communication and exchange with each other and with the surrounding. Higher-resolution molecular models show how similar complexity is present also in realistic supramolecular polymer systems. This multiscale approach reveals the dynamic nature of supramolecular polymers, providing an exquisitely statistical view of these complex molecular systems.

\section{Results and discussion}

\section{Dynamic equilibrium in a supramolecular system}

In systems that self-assemble in solution, the formed assemblies reach a dynamic supramolecular equilibrium, characterized by continuous exchange of molecular units among assemblies and with the environment (Figure 1a). To explore the factors that control such complex dynamics, we here employ a minimalistic model of a supramolecular polymer system, constituted by monomeric units that mutually interact in a directional, short-ranged and reversible way, spontaneously polymerising into one-dimensional fibres during MD simulations (Figure 1).

The monomers (M) are composed of 7 beads (Figure 1b) disposed in a planar hexagonal shape (see Methods and Supporting Information, SI, for details), and interact mostly via the central beads, which are strongly attracted to each other. The six lateral 
beads are instead weakly interacting and, shielding the attraction between core beads at the monomer sides, impart directionality to the self-assembly. The non-bond interactions between beads are determined by Lennard-Jones (LJ) potentials, with fixed radius $(\sigma)$ for all the beads, and two different values of interaction strength $(\varepsilon)$ : large (several $k_{B} T \mathrm{~s}$ ) for the interaction between central beads, and small (a fraction of $k_{B} T$ ), when lateral beads are involved (Figure 1b). The dynamics of the system is modelled via Langevin equations of motion. The effect of the solvent is represented implicitly, i.e. the non-bond potential between the beads accounts for both solute-solute and solute-solvent interactions, while friction and fluctuations are rendered by Langevin dynamics. As the interaction between monomers is mainly governed by directional, specific attraction, this model mimics supramolecular polymers where (i) monomers self-assemble in a good solvent, and (ii) the monomer shape and features favor mutual directional aggregation. ${ }^{48}$

We simulated $500 \mathrm{M}$ monomers in a fixed cubic volume, at fixed temperature ( $T=300 \mathrm{~K})$. Starting from randomly distributed monomers, one-dimensional fibres spontaneously formed during CG-MD (Figure 1c), reaching an equilibrium that depends on the stacking attraction between monomer cores (the interaction strength $\varepsilon$ between the central beads). We first compared CG-MD simulations of the same system $\left(\varepsilon=40 \mathrm{~kJ} \mathrm{~mol}^{-1}\right)$, starting from two different configurations (Figure 2a), where the $500 \mathrm{M}$ monomers are either randomly dispersed/disassembled (R) or pre-stacked into 20 equally-sized fibres (S). After a transient phase of the order of $\mu$ ss both simulations converge to the same equilibrium state.

Figure $2 b$ shows that, at the equilibrium, molecules are continuously exchanged between assemblies, even after the size-distribution has converged (insets of Figure $2 b$ ). The average coordination between monomer centers $(\phi)$, indicates the stacking order of the system ( $\phi=2$ signals perfectly stacked infinite fibres). Independently of the starting condition, the system converges to the same average structural/stacking order (Figure 2d,top).

The dynamic nature of the supramolecular equilibrium state, qualitatively shown in Figure $2 b$, is quantitatively analysed in Figures 2c-f. We e.g report the molecular traffic and flux ${ }^{54}$ along CG-MD (figures $2 \mathrm{~d}$ (bottom), $2 \mathrm{f}$ (top), S3 S5, S7): the traffic indicates the sum of molecular binding and unbinding events (per monomer) during the CG-MD, while the flux indicates the cumulative difference between these binding and unbinding events (see Methods for details). After the equilibration stage ( $\sim 1 \mu$ s of CG-MD), the flux reaches a plateau while the traffic keeps increasing (see Figure $2 \mathrm{f}$ ). This means that, at the equilibrium, the average size of aggregates remains stable (binding and unbinding events balance), while monomers/oligomers continuously exchange among different constructs. This manifests the intrinsically dynamic character of these systems at the equilibrium. Both $\mathrm{S}$ and $\mathrm{R}$ cases converge to the same traffic and flux profiles, indicating that they have reached the same dynamical equilibrium state.

These are averaged results, which pertain to the entire solution, but the same holds also at a microscopic level, relatively to the size of individual assemblies that populate the system (Figure 2e). After $\sim 1 \mu$ s of CG-MD, the assembly populations converge to nearly identical size distributions in both $\mathrm{S}$ and $\mathrm{R}$ cases, as these systems lose memory of the initial conditions and converge to the same dynamical equilibrium state. The dynamics of this model should be compared carefully with experiments. The minimalistic molecular representation and large monomer concentration of the simulations $(\sim 77 \mathrm{mM} v s$. typical experimental concentrations in the $\mu \mathrm{M}$ scale) may impact the self-assembly pathway, ${ }^{38}$ the size-distribution at the equilibrium, etc. Nonetheless, these models can demonstrate how changing key parameters affects the supramolecular dynamics, providing useful qualitative trends.

To investigate how the monomer-monomer interaction strength affects such collective structural/dynamical equilibrium, we compared simulations with increasing interaction strength $(\varepsilon)$ between the monomer core beads: $\varepsilon=40 \mathrm{~kJ} \mathrm{~mol}^{-1}, \varepsilon=$ $45 \mathrm{~kJ} \mathrm{~mol}^{-1}$ and $\varepsilon=50 \mathrm{~kJ} \mathrm{~mol}^{-1}$. As shown in Figure $2 \mathrm{~b}$, at the dynamical equilibrium state the assembly size-distribution is globally conserved (insets), while molecular entities are continuously exchanged between the assemblies (details on the equilibration are reported in Figures S2-S10).

The data show how changing the $\varepsilon$ can entail a different equilibrium structure and dynamics (Figures $2 \mathrm{~b}, \mathrm{c}, \mathrm{f}$ ). The size distributions of Figure $2 \mathrm{c}$ indicate that longer fibres are formed when the directional interaction between the monomers is stronger. At the same time, the evolution of traffic shows that the the systems are increasingly dynamic when $\varepsilon$ is decreased, even after the equilibrium is reached, as signaled by the convergence of the molecular flux (Figure 2f).

Thus, our data support a general trend: increasing the monomer-monomer interactions produces longer fibres, but reduces the molecular exchange at the equilibrium (Figures 2c,f, and S2-S12). Such behavior fits qualitatively well with recent observations on, e.g., BTA systems, where increasing the number of carbon atoms in the monomer side-chains (favoring their self-assembly in aqueous environment) produces more persistent and less dynamical fibres in water. ${ }^{16,19,44,55,56}$

\section{Molecular communications in supramolecular polymer systems}

The equilibrium dynamics of supramolecular systems involves a continuous exchange of molecular entities. Relevant questions concerning such communication between assemblies are: do all fibres exchange the same kind of fragments? Or do they communicate in different ways (e.g., exchanging different species) depending on their size? In general, what factors control this inter-assembly communication? 
a
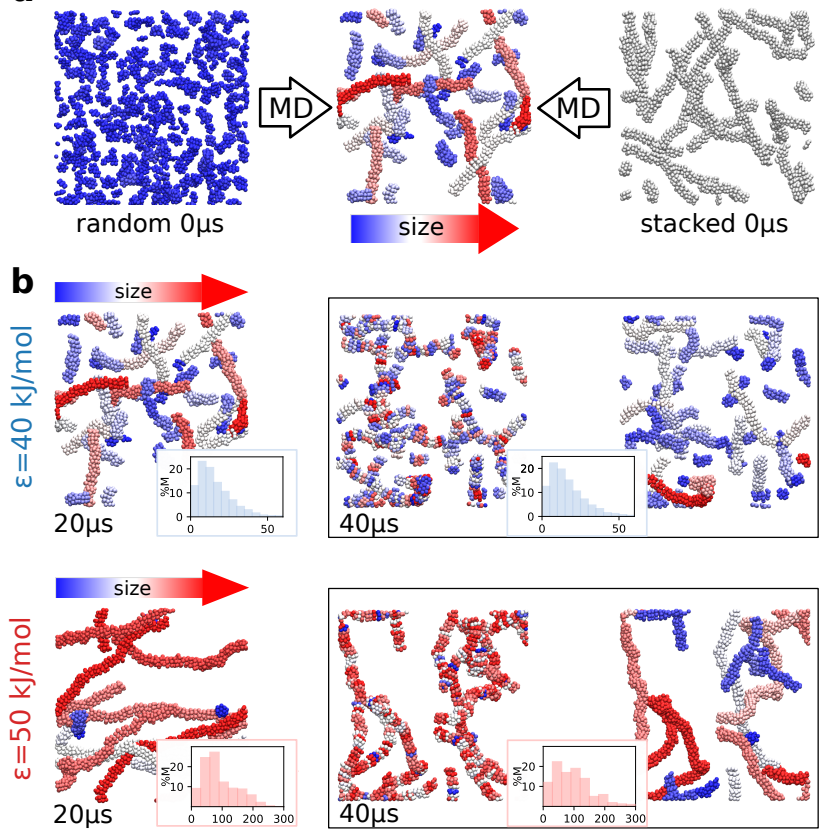
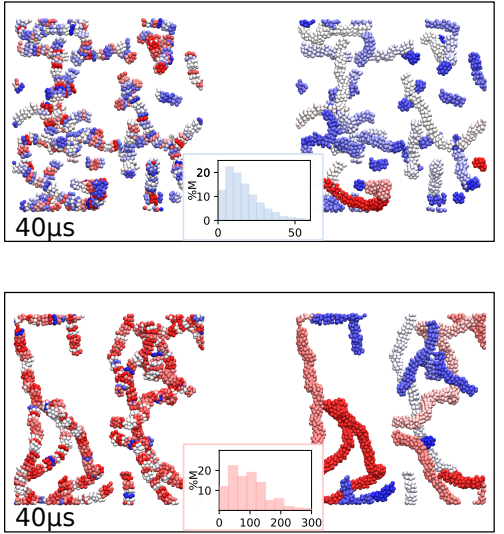

C

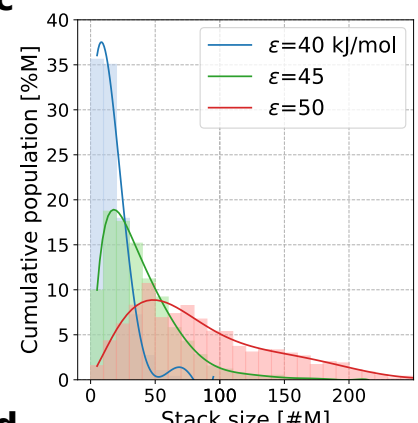

d
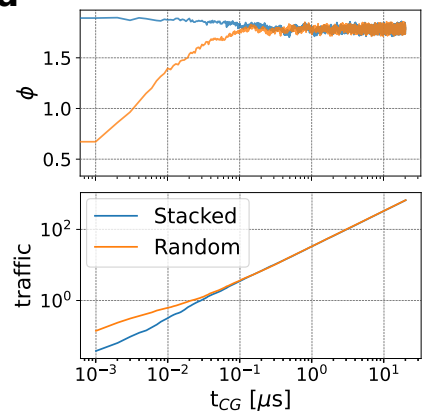

e
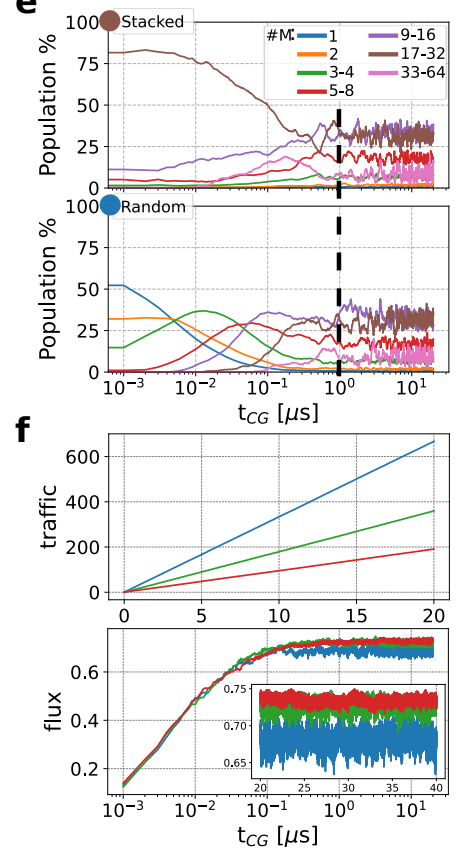

Figure 2. Dynamic equilibrium in a minimalistic self-assembling model. (a) CG-MD simulations of a model system composed of $500 \mathbf{M}$ monomers, starting from randomly dispersed monomers (R case, left) or monomers pre-stacked into 20 equal fibres (S case, right). During CG-MD both systems relax to the same dynamical equilibrium state (center). The assemblies are colored based on their size (see color bar). (b) CG-MD snapshots for the $\varepsilon=40 \mathrm{~kJ} \mathrm{~mol}^{-1}$ (top) and $\varepsilon=50 \mathrm{~kJ} \mathrm{~mol}^{-1}$ systems (bottom). Left: snapshots at $t_{C G}=20 \mu \mathrm{s}$ (assemblies colored according to their size). Center: snapshots at $t_{C G}=40 \mu \mathrm{s}$, where the monomer coloring of $t_{C G}=20 \mu \mathrm{s}$ (left) is preserved - the color reshuffling indicates molecular exchange after equilibrium is reached. Right: same snapshots as center but assemblies colored according to their current size (at $t_{C G}=40 \mu \mathrm{s}$ ). The relative assembly-size distributions are reported in the insets. (c) Average distribution of aggregate sizes for different $\varepsilon$ values at the equilibrium. (d) Average coordination number $\phi$ between the $\mathbf{M}$ centres (top) and cumulative molecular traffic ${ }^{54}$ (bottom) along CG-MD in the $\mathrm{S}$ and $\mathrm{R}$ cases. (e) Evolution of the assembly-size populations for the $\mathrm{S}$ (top) and $\mathrm{R}$ (bottom) cases. The brown and blue circles at $t_{C G}=0$ indicate the initial population in the two cases. After $t_{C G}=1 \mu \mathrm{s}$ (vertical dashed black line) the two systems reach a comparable microscopic equilibrium state, and the populations plateau. (f) Molecular traffic and flux ${ }^{54} v s$. time at different $\varepsilon$ values. 
To study in detail what species are exchanged among the fibres at the equilibrium, we monitored the assembly (size) to which each monomer belongs to at each step, and tracked their transitions every $\Delta \tau$ time interval. We analysed in this way the CG-MD trajectories at equilibrium $\left(t_{C G}=20-40 \mu \mathrm{s}\right)$ with a sampling time interval of $\Delta \tau=300 \mathrm{ps}$, frequent enough to reduce the probability of assemblies undergoing multiple binding/unbinding events during $\Delta \tau$ (see Methods for details). We thus obtained transition matrices (Figure 3a, centre) counting how many times a monomer is exchanged from an assembly of size $i$ (matrix row index) to another of size $j$ (column index). Normalizing the matrix rows to 1 , we obtain transition probability matrices: the numbers on the diagonal are indicative of the probability that a monomer inside an assembly of size $i$ remains in that assembly during a $\Delta \tau$ interval. The off-diagonal entries are indicative of the probability that a monomer in an assembly of size $i$ is involved in a transition, becoming part of a larger $(i$-to- $j$, where $j>i)$ or smaller $(j<i)$ assembly during $\Delta \tau$ (Figure 3a, left and right).

Figure 3 reports the transitions detected for the $\varepsilon=45 \mathrm{~kJ} \mathrm{~mol}^{-1}$ system (see SI for data on the other systems and additional details). Two zoomed regions of the transition probability matrix are reported in Figure $3 \mathrm{a}$ (left and right panels), identifying respectively the dynamics of smaller or larger aggregates. As summarized in the scheme of Figure $3 \mathrm{~b}$, the off-diagonal pathways on the right (red arrow) signal polymerisation events, while the left ones (blue arrow) signal depolymerisation events. Noteworthy, at the equilibrium, small assemblies are more likely to aggregate into larger assemblies, rather than to disassemble - the probabilities are larger on the right-side of the diagonal (Figure 3a, left). This is the case up to assemblies of size $\sim 33-64$ monomers for the $\varepsilon=45 \mathrm{~kJ} \mathrm{~mol}^{-1}$ system (but which depends on the $\varepsilon$ value). As the range increases, the fibres become inherently less stable, and fragmentation becomes more likely than growth (Figures 3a:right and $3 b$ ). The tendency to self-assembly is thus contrasted by the increasing probability of fragmentation. These competing factors determine the dynamic equilibrium in the monomer exchange. When $\varepsilon$ is decreased $\left(40, \mathrm{~kJ} \mathrm{~mol}^{-1}\right)$, the equilibrium shifts to smaller aggregate sizes (Figure S14a: highest probability for $\sim 5-16$ monomers). Vice versa, for stronger monomer-monomer interactions $\left(\varepsilon=50, \mathrm{~kJ} \mathrm{~mol}^{-1}\right.$ ) the equilibrium moves towards longer fibres (Figure S14b: highest probability for $\sim 65-128$ ). We refer to (logarithmic) ranges of fibre sizes, as we aimed at a qualitative picture of how the equilibrium dynamics shapes the entities that are most probably observed (exact length values are less meaningful in such fluctuating complex systems).

Comparing the transition matrices of the three systems, we also observe that, as the $\varepsilon$ increases, it becomes less likely to observe monomers and small aggregates. At $\varepsilon=40 \mathrm{~kJ} \mathrm{~mol}^{-1}$, the disassembled monomers have a $\sim 60 \%$ probability to remain isolated at every $\Delta \tau$ (Figure S14a:left). The probability drops to $\sim 52 \%$ and $\sim 49 \%$ for the systems with $\varepsilon=45$ and $\varepsilon=50 \mathrm{~kJ} \mathrm{~mol}^{-1}$ cases (Figures 3a:left and S14b:left, respectively).

Overall, these transition matrices (Figures 3a and S14) provide also insights into the prevalent mechanisms by which the assemblies exchange molecular units/fragments, communicating with each other. Comparing between the different systems, we can assess how changes in the system parameters may transform such communications.

The fibres can either elongate by acquiring monomers (or oligomers), or by coalescence with similar-size assemblies. Similarly, fibres depolymerisation may occur via monomer (or oligomer) exchange with the solution, or via fragmentation into shorter fibres. The scheme of Figure $3 \mathrm{~d}$ identifies those regions of the transition matrix (i.e. 3c) that refer to such different classes of events: involving the exchange of small size aggregates (yellow and green areas) or the coalescence/fragmentation of medium/large constructs (red and blue areas). For simplicity, the representation in Figure 3d shows only "binary" events, neglecting those cases in which a fibre fragments into more than two sections, or multiple fragments combine into a single assembly. These events can be considered rare given the short sampling interval $\Delta \tau$ (see SI for details). We quantified the statistical relevance for the different mechanisms represented in Figure 3d. To this end, we first chose two characteristic aggregate sizes that delimit the matrix areas in the scheme: the first, $A$, discerns between medium and large assemblies, and is chosen as the average aggregate size at the equilibrium $A=\langle$ size $\rangle:\left(\right.$ e.g., $\sim 21$ monomers in the $\varepsilon=45 \mathrm{~kJ} \mathrm{~mol}^{-1}$ system $)$. The second parameter, $E=\langle$ size $\rangle / 5 \sim 4$, distinguishes between medium and small assemblies (Figure 3d). Using these parameters, we observe that, for the $\varepsilon=45 \mathrm{~kJ} \mathrm{~mol}^{-1}$ system, $\sim 58 \%$ of the total growth events consists in the coalescence/fusion of large fibres (size $>A$, Figures $3 \mathrm{~d}$ and 3e: blue area). In a similar way, we calculated that $\sim 18 \%$ of the events consist in the elongation/shortening of smaller fibres (of size $<A$ ) via binding/unbinding of monomers (or small oligomers of size $<E$ : yellow area of Figure 3e). Elongation/shortening of long fibres (size $>A$ ) via binding/unbinding of monomers/small oligomers impacts for $\sim 14 \%$ of all the events (Figure 3e: green area), while $10 \%$ of transitions involves the coalescence/fragmentation of medium-size fibres $(E<$ size $<A$, red area). While the blue areas contribute the most to the dynamics of this system, also events involving small/medium species appear with relevant probabilities. While the weight of different mechanisms may change depending on the choice of $A$ and $E$, this analysis provided rather robust evidence that all four mechanisms shown in Figure $3 \mathrm{~d}$ contribute substantially to the system dynamics (see SI for additional details).

We also observed that the exchange of monomers/oligomers between the fibres and the environment occurs mostly via the fibre tips. Exchange of monomers/oligomers from the fibre-bulk was detected with an occurrence of $<0.001 \%$ of the binding/unbinding events. Generating well-ordered fibres via directional interactions (negligible solvophobic interactions), the M model mimics the case of supramolecular polymers in a good solvent, where molecular exchange occurs preferentially from 
a

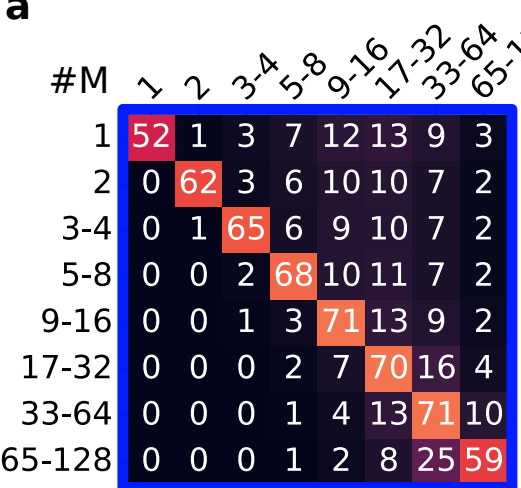

b
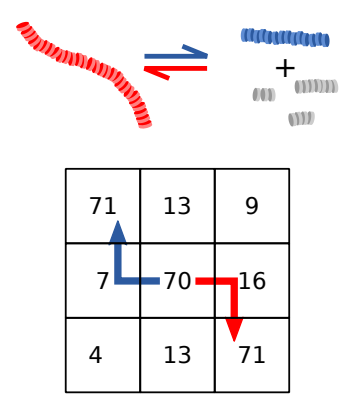

e

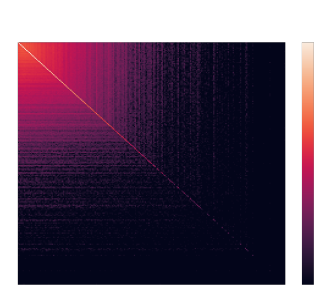

$\varepsilon=45 \mathrm{~kJ} / \mathrm{mol}$

RAW TRANSITION DATA

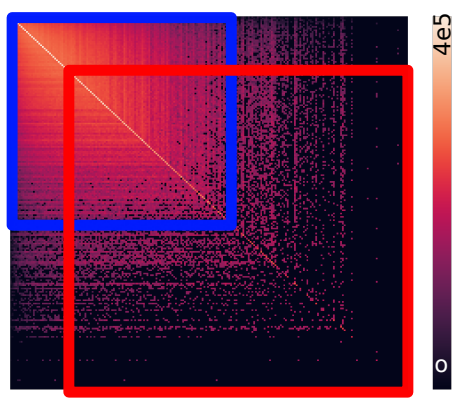

C

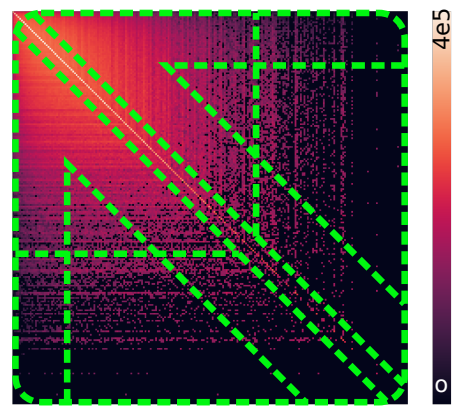

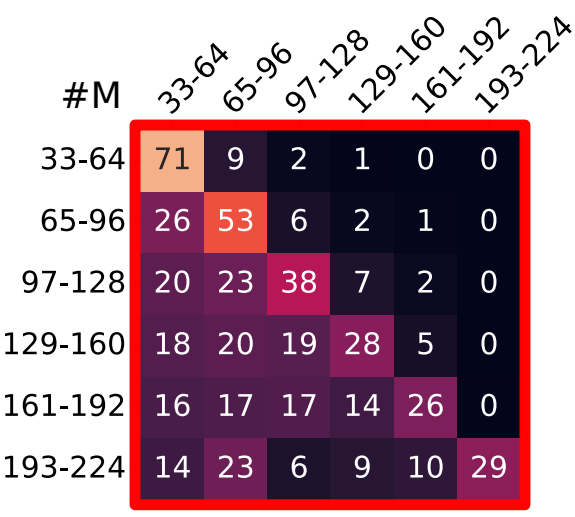

d

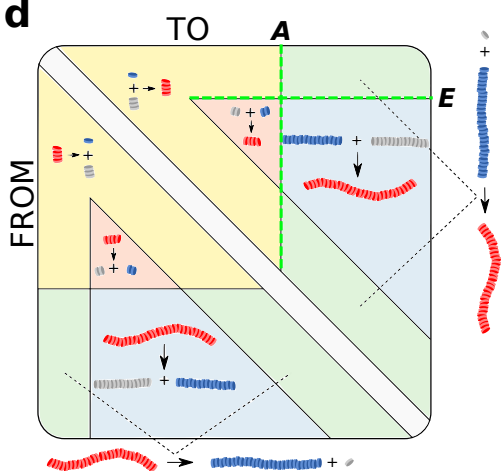

$14 \%$

$58 \%$
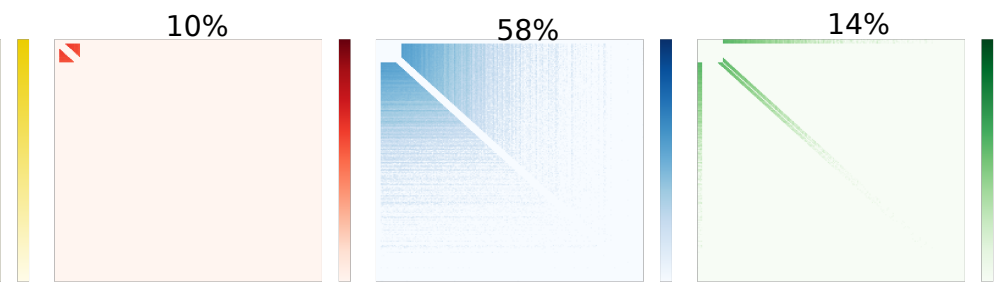

f

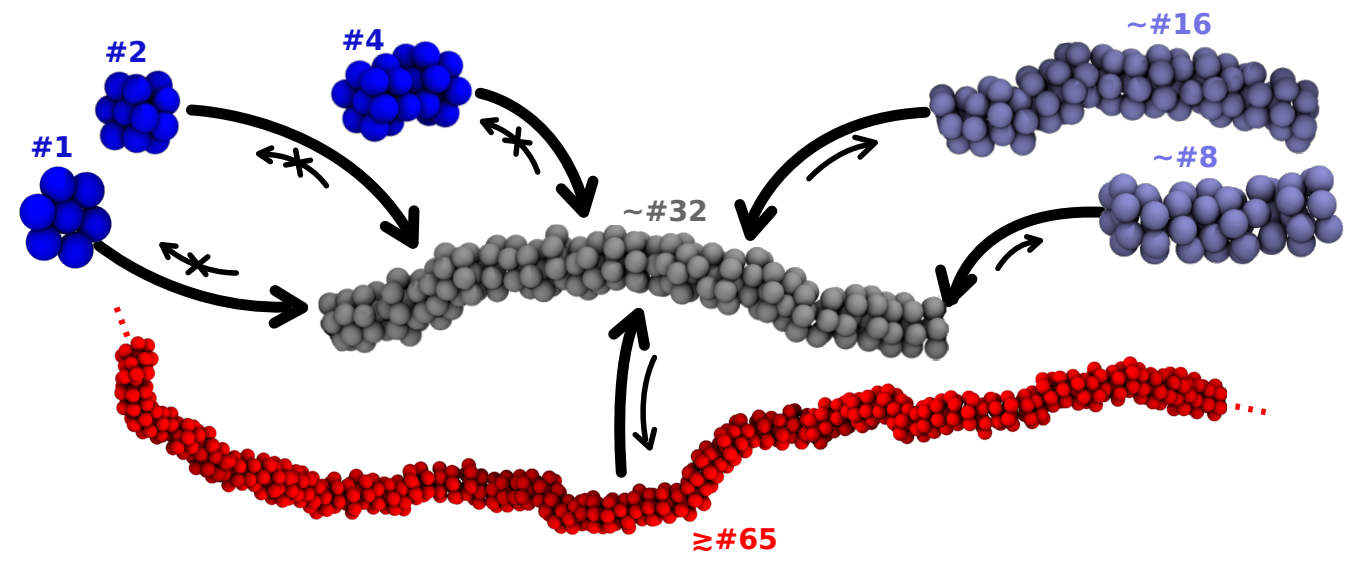

Figure 3. The dynamic nature of a supramolecular polymer system. Results are reported for the $\varepsilon=45 \mathrm{~kJ} \mathrm{~mol}^{-1}$ system (other cases are reported in the SI). (a) The transition matrix obtained from CG-MD with sampling interval $\Delta \tau=300 \mathrm{ps}$ (center). The left and right panels report two sub-regions of the transition probability matrix (red and blue rectangles). Here, the size of the aggregates are grouped for clarity. The numbers in the cells indicate the percentage probability (the 0s identify transitions with probability $\leq 0.5 \%$, see Figure S13). The coloring of the matrix cells mirrors the entry values (with logarithmic scale for the raw transition data matrix). (b) Illustrative scheme interpreting the transition matrices in terms of polymerisation (red) and depolymerisation (blue arrow) events. (c) Matrix partitioned in areas indicating the different polymerisation/depolymerisation mechanisms.(d) Diagram associating the different polymerisation/depolymerisation mechanisms to the different regions of the transition matrix. (e) Matrix counting the assembly transitions (left) decomposed into areas (as in d) identifying different classes of polymerisation/depolymerisation mechanisms (see Methods for details).(f) Dynamic interconnections between a 32 monomer aggregate (gray) and smaller (blue) or larger assemblies (red). 
the fibre tips. Monomer exchange from the fibre bulk is instead typical of systems dominated by non-directional/solvophobic effects, where defects along the fibres govern the monomer dynamics. ${ }^{44,48}$

From the transition probabilities it is possible to build diagrams of the dynamic interconnections between assemblies in these systems (see e.g., Figure 3f). The results obtained with such a minimalistic model highlight the complexity of the dynamic equilibrium in elementary supramolecular systems. It is interesting to investigate if such complexity is encountered also in more realistic supramolecular polymer models, and what aspects are unavoidably over-simplified and overlooked.

\section{Analogies with realistic supramolecular polymer models}

To increase the chemical relevance of our study, we then compared the behavior of the $\mathbf{M}$ model with a higher-resolution supramolecular polymer model. Building on our simulations on BTA supramolecular polymers, ${ }^{37,38,44,57}$ we recently developed a CG model that, while being consistent with BTA behavior, is representative of a larger class of supramolecular polymers, formed by monomers with three symmetric side-chains connected to a stacking centre. ${ }^{48}$ In this CG model, the stacking center is composed of a central bead and a charged dipole. The dipoles of different monomers interact with each other, reproducing a directional interaction consistent with that of BTA motifs. Three flexible side-chains, each composed of five CG beads (Figure 4a,bottom) are symmetrically connected to the monomer centre. The side-chains beads are solvophilic, so that this model is consistent with BTA supramolecular polymers (and similar supramolecular motifs) in a good solvent (similarly to the $\mathbf{M}$ minimalistic model described above) ${ }^{48}$ Initially developed as explicit solvent model, ${ }^{48}$ we here re-parametrized this CG model to behave consistently in implicit solvent (see Methods and SI for details). This allowed us to simulate a large number of monomers for relatively long timescales, obtaining results comparable to those obtained with the $\mathbf{M}$ model. In the following we will refer to this model as "BTA".

The BTA monomers are intrinsically different from the $\mathbf{M}$ units. In fact, while the flat symmetry and directional interaction between the BTA cores produce an ordered monomer stacking as in the $\mathbf{M}$ model, the higher-resolution description of the BTA monomers accounts (to some extent) for the effect of molecular flexibility (whereas the $\mathbf{M}$ units are rigid). Investigating how similar is the supramolecular equilibrium of the BTA model to that of the $\mathbf{M}$ model could provide insights on how molecular flexibility impacts the dynamics of the assembled system. Also, it could reveal to what extent the complexity of a realistic system is lost when considering a minimalistic representation such as that of $\mathbf{M}$ model.

Also in this case, we simulated the self-assembly of 500 initially dispersed BTA monomers in a cubic simulation box, such that the monomer concentration was identical to that of the $\mathbf{M}$ simulations (see Methods). In this case we conducted CG-MD simulations at three different temperatures: $T=300 \mathrm{~K}, T=320 \mathrm{~K}$ and $T=340 \mathrm{~K}$, as increasing/decreasing the temperature has the effect of weakening/strengthening the self-assembly propensity. Similarly to the $\mathbf{M}$ model, the BTA monomers reached the equilibrium forming well-ordered fibres of diverse sizes during CG-MD (Figure 4b, right). The CG-MD trajectories of these simulations were then analyzed as done for the $\mathbf{M}$ model, comparing the structural and dynamical behavior of the different models at the equilibrium.

Figure $4 \mathrm{c}$ shows that, at the equilibrium, similarly-sized aggregates populate the system in the BTA-like system at $T=320 \mathrm{~K}$ and the $\mathbf{M}$ system with $\varepsilon=45 \mathrm{~kJ} \mathrm{~mol}^{-1}$ (see Figure S20 for the other systems). This similarity is confirmed by Figure $4 \mathrm{~d}$, showing the average percentage of different-size assemblies that populate the systems in equilibrium conditions (see also Figures 4d, S18 and S19).

Comparing these simulations also demonstrates that changing the temperature in the BTA systems has similar effect on the aggregate-size distribution than varying $\varepsilon$ in the $\mathbf{M}$ model. Increasing the BTA system temperature to $340 \mathrm{~K}$ generates an equilibrium size distribution more similar to that obtained in the $\mathbf{M}$ system at $\varepsilon=40 \mathrm{~kJ} \mathrm{~mol}^{-1}$ (Figure $4 \mathrm{~d}$ ). In both cases, relatively small aggregates (average size $<10$ monomers) are observed, with significant free-monomer populations, up to $\sim 34 \%$ BTA case and $\sim 6 \%$ for the $\mathbf{M}$ model (more comments on this aspects in the next section). Although these model systems are considerably over-concentrated compared to realistic dilute conditions, thus favoring monomer aggregation, these data suggest a fairly limited tendency to self-assembly. These results are in qualitative agreement with experimental evidences showing a critical transition temperature for, e.g., the BTA motif at $\sim 70^{\circ} \mathrm{C}(\sim 343 \mathrm{~K})$, above which BTA self-assembly is hindered. ${ }^{37,48,56}$ Analogous qualitative similarities hold also when the BTA model at $T=300 \mathrm{~K}$ and $\mathbf{M}$ model with $\varepsilon=50 \mathrm{~kJ} \mathrm{~mol}^{-1}$ are compared, as the spontaneous formation of longer fibers is favored in both cases (Figure 4d).

The molecular traffic and flux data of Figure 4e show that, globally, the temperature variation affects also the equilibrium dynamics of the BTA systems similarly to what the variation of $\varepsilon$ does in the $\mathbf{M}$ system. In general, the collective behavior of these two models exhibits significant analogies on an ensemble (average) level. However, deeper-level questions arise when comparing the $\mathbf{M}$ and BTA systems at a microscopic level: how similar is the molecular exchange and communication dynamics among the self-assembled fibres in these two models of complex systems?

\section{Mechanisms of communication between supramolecular polymers}

From the CG-MD simulations, we computed the transition matrices for the BTA systems in the same way as we proceeded for the $\mathbf{M}$ ones. From Figures 5a,b, it appears clear that analogies are observed also in what pertains the molecular exchange 
a

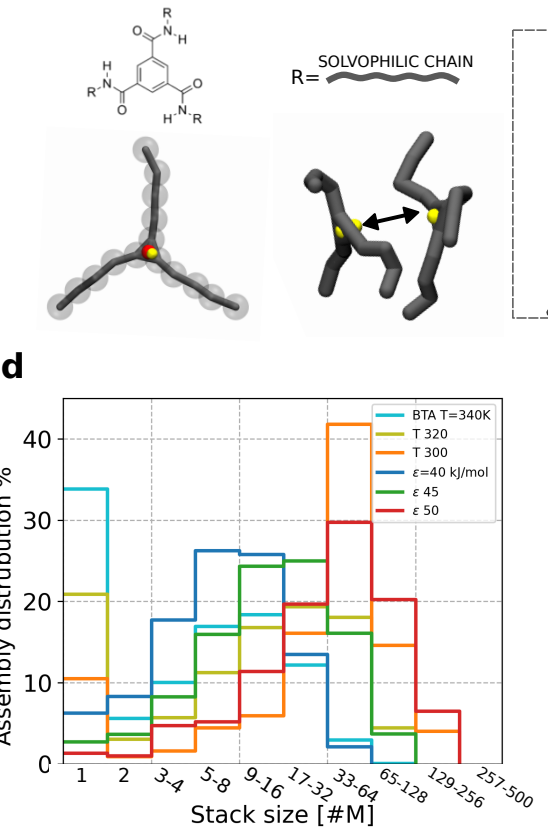

b

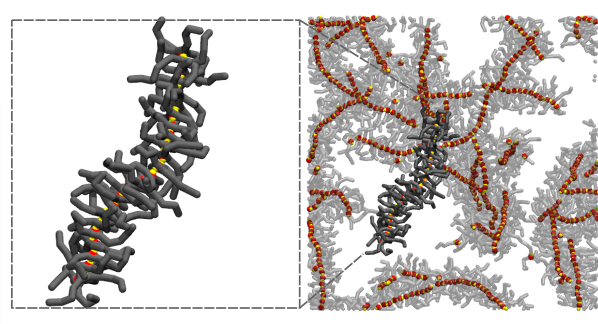

e

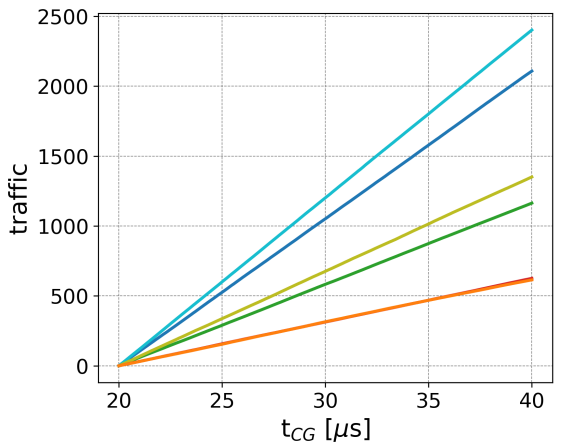

C
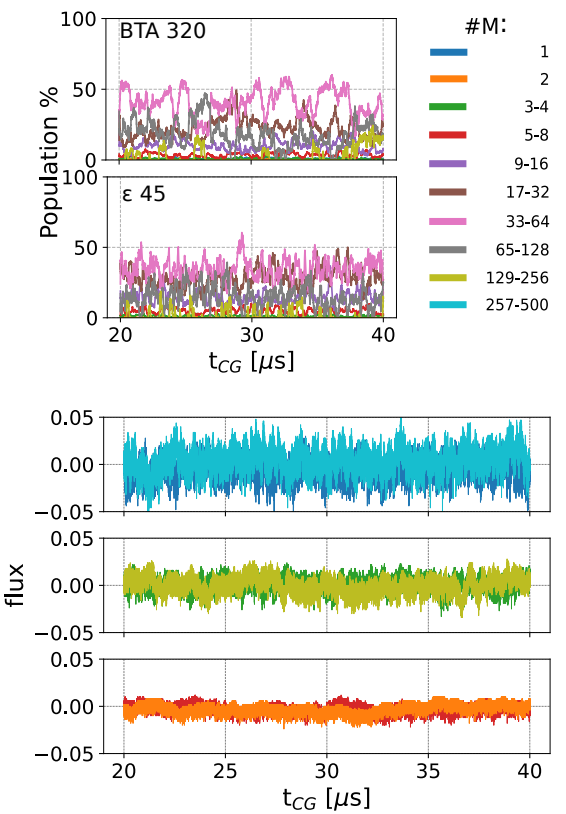

Figure 4. Coarse-grained model of self-assembling supramolecular polymers. (a) Molecular structure of a BTA monomer core (top), which can be decorated with generic (e.g., solvophilic) side chains. Bottom: CG model of a BTA-like solvophilic monomer (left), which interacts directionally with monomers of the same specie (right). (b) Snapshot of BTA fibres formed spontaneously after $t_{\mathrm{CG}}=20 \mu \mathrm{s}$ of CG-MD, starting from 500 dispersed BTA monomers. A single fibre is highlighted and reported in the frame on the left. (c) Time evolution of the assembly-size populations (in percentage of monomers) for the BTA model at $T=320 \mathrm{~K}$ (top) and $\mathbf{M}$ model with $\varepsilon=45 \mathrm{~kJ} \mathrm{~mol}^{-1}$ (bottom) at the equilibrium. (d) Distribution of assemblies of different sizes (percentage over the average number of assemblies) for different BTA and $\mathbf{M}$ systems. (e) Cumulative molecular traffic (left) and flux (right) ${ }^{54} v$ s. time for different BTA and $\mathbf{M}$ systems at the equilibrium. The colors are the same of (d). 
between the assemblies. Comparing the $\mathbf{M}$ system with $\varepsilon=40 \mathrm{~kJ} \mathrm{~mol}^{-1}$ and the BTA system at $T=340 \mathrm{~K}$, we observe that the transition matrices look qualitatively similar. Peculiar, yet relevant differences can be nonetheless noticed. In particular, the free disassembled monomers at the equilibrium are more persistent in time in the BTA system with respect to the M system. Observing free monomers in solution is relatively more likely in the BTA than in the M systems (Figure 4d, 5a,b). Noteworthy, this does not mean that the self-assembly is less favored in the BTA system than in the M model - the probability associated to larger assemblies is rather similar in the two systems (e.g., $~ 73 \%$ for $17-32$ monomers in the $\mathbf{M}$ system with $\varepsilon=40 \mathrm{~kJ} \mathrm{~mol}^{-1} v$ s. $\sim 69 \%$ in the BTA system at $\left.T=340 \mathrm{~K}\right)$. In conditions where monomer self-assembly is more favored (e.g. M with $\varepsilon=50 \mathrm{~kJ} \mathrm{~mol}^{-1}$ and BTA at $T=300 \mathrm{~K}$ ), large fibre sizes are even more persistent in time in the BTA than in the $\mathbf{M}$ system, while the same holds also for the monomers. This suggests that the free-energy landscape for monomers self-assembly differs between the BTA and the $\mathbf{M}$ system. In particular, consistent with the experimental evidence, the BTA systems show the characteristic behavior of cooperative self-assembly, where a critical-size nucleus must be overcome to self-assembly, and where the presence of monomers along with longer fibers is expected at the equilibrium. ${ }^{25,31,37,58-60}$ In this perspective, the assembly-size distribution in Fig. $4 \mathrm{~d}$ and probability matrices in Figure 5a,b depict $\mathbf{M}$ as a predominantly isodesmic system. The increased survival of disassembled monomers can be imputed to the flexible structure of the BTA monomer model. In such a higher-resolution model, the side-chains can wrap around the BTA centers, minimising the residual solvophobicity of the monomer (cores), and generating a globular and isotropic solvated state of the monomers. Such effect is absent in the $\mathbf{M}$ monomers due to their flat and rigid structure. Such a difference between the two models becomes less relevant when aggregates are considered (negligible for size $>3-5$ monomers), as in larger BTA assemblies the monomers can optimise their interactions collectively, forming ordered conformations in which the side-chains are extended and do not interfere with the core-core interactions. ${ }^{31,37}$ Such evidence allows us to draw the following considerations. First, in such systems the cooperativity in the self-assembly seems to emerge from competing effects: the interaction between the monomers, favoring self-assembly, vs. the flexibility of the molecular structure, which determines differences between the configuration of monomers in the disassembled and in the assembled states, due to molecular movements and to the presence of residual (even minimal) solvophobic effects. Second, in the case of rather complex and flexible monomers, a minimalistic description of their structure may determine an over-approximated representation of self-assembly, impairing a correct modeling of cooperativity in self-assembly.

Figure $5 \mathrm{~b}$ reports the transition matrices of the two model systems with the strongest self-assembling drive, namely the Ms at $\varepsilon=50 \mathrm{~kJ} \mathrm{~mol}^{-1}$ and the BTAs at $T=300 \mathrm{~K}$. We note that the molecules/fragments are exchanged between the various size assemblies in a similar way in both systems. In these conditions, the monomers (when they do not remain disassembled) tend to flow toward the most stable aggregates in the systems (33-64 and 65-128-mers). At larger sizes, this is again contrasted by the fiber tendency to fragmentation. Analogous similarities hold also for the cases of $\mathbf{M}$ with $\varepsilon=45$ or $\varepsilon=40 \mathrm{~kJ} \mathrm{~mol}^{-1}$ and BTA at $T=320$ or $T=340 \mathrm{~K}$ respectively (see Figure 5a, and Figure S24 in the SI).

We then quantified the statistical weight of the different exchange mechanisms in the global equilibrium dynamics of the systems (see Methods for details). We classified the events tracked in the matrices as done in Figure $3 \mathrm{e}$ (to ease the comparison, we used the same threshold sizes $A$ and $E$ defined for the $\mathbf{M}$ model, also for the BTA systems, complete data in Figure S25 in the SI). To simplify the analysis, we now group all the exchange events under two main inter-assembly communication mechanisms: (i) via exchange of small entities $($ size $<E)$ and fragmentation/coalescence of medium-size aggregates $(E<\operatorname{size}<A$ ), or (ii) via fragmentation or coalescence of large molecular entities (of size $>A$ ). Referring to the illustrative diagram of Figure 3d, (i) encompasses all events in the yellow, red and green areas, while (ii) identifies the events pertaining to the blue area. According to such a classification, this analysis estimates that $\sim 74 \%$ of the exchange events in the BTA system at high-temperature $(T=340 \mathrm{~K})$ involves only monomers and oligomers, while inter-fibres communication via fragmentation/coalescence contributes by $\sim 26 \%$ (see Figure $5 \mathrm{c}$ : gray $v s$. blue columns in the inset). Decreasing the temperature to $T=320 \mathrm{~K}$, we observe an intermediate behavior. Under such conditions, the dynamics of the system is controlled by exchange of both small $(\sim 60 \%)$ and large species ( $\sim 40 \%$ - see Figure S25 in the SI). The exchange of monomers and smaller fragments becomes then less relevant in the inter-fiber communications at $T=300 \mathrm{~K}$ for the BTA system. In such conditions, our simulations indicate that small exchanging species give a minor contribution to the molecular communication in the system, while fragmentation and fusion of longer fibres become dominant (Figure 5d: gray vs. blue columns at $\sim 17 \%$ vs. $\sim 83 \%$ respectively). A similar scenario is observed also for the $\mathbf{M}$ system with $\varepsilon=50 \mathrm{~kJ} \mathrm{~mol}^{-1}$ (Figure S25). Additional tests changing the threshold parameters used for these analyses demonstrate the robustness of the obtained results and the general validity of such evidence (see Figure S27 in the SI).

The Ms and BTA systems appear similar also for what pertains to the dominant mechanisms of communication between assemblies in the systems. While the statistical weights discussed above may depend, to some extent, on the molecular concentration, the obtained trends provide solid, general-level insights. Our results reveal that the dominant mechanisms by which the fibres communicate in such complex systems may change as the interaction strength between the monomers varies (either by changing the $\varepsilon$ or the $T$ ). This is captured in a similar way by both the minimalistic, rigid $\mathbf{M}$ model and by the 

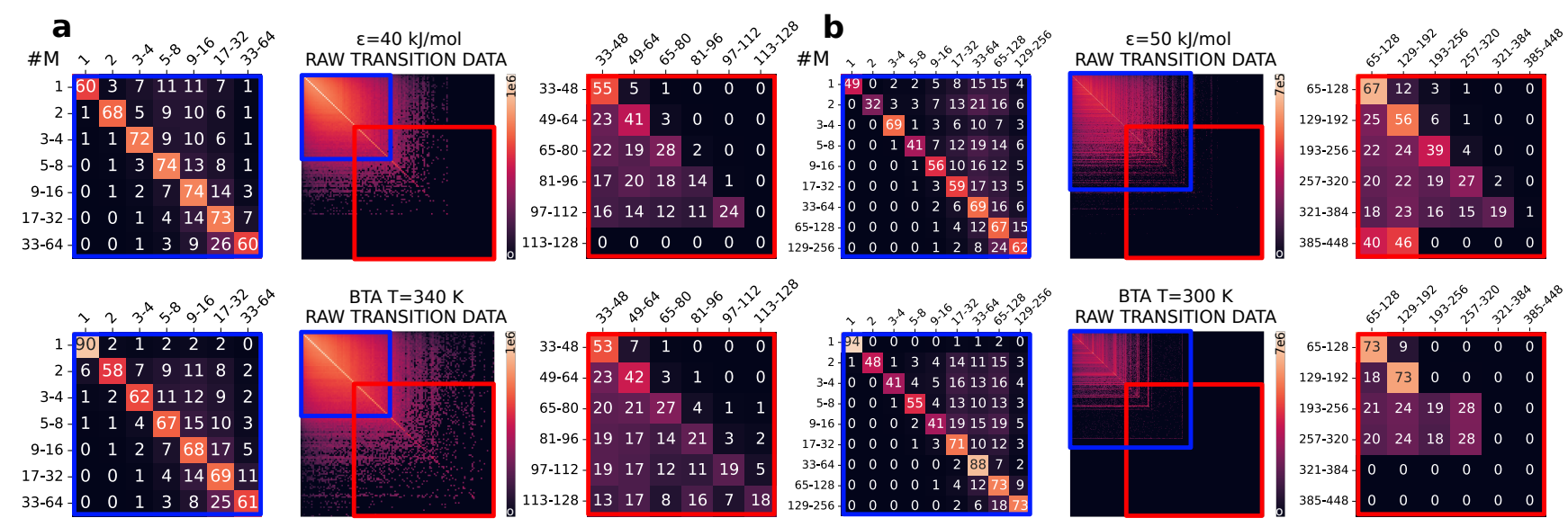

\section{c}
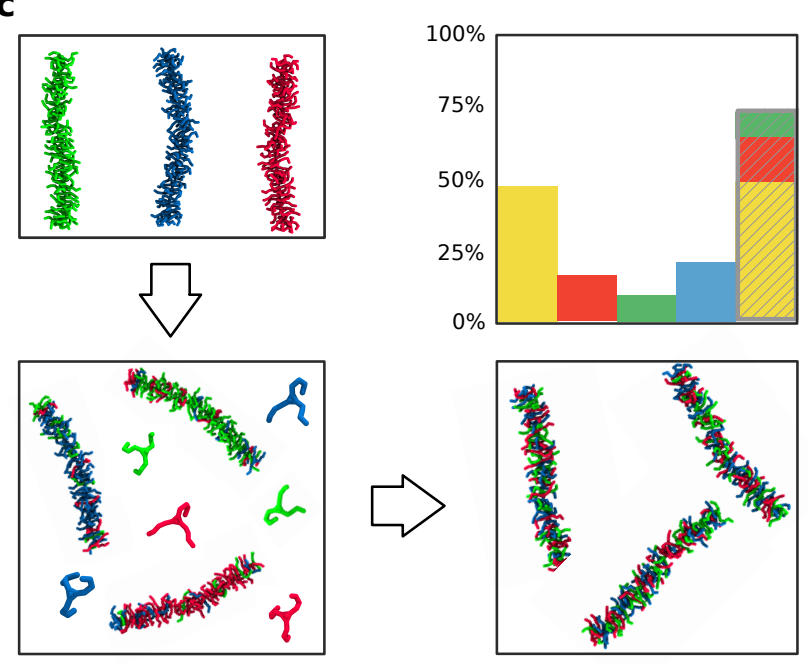

d
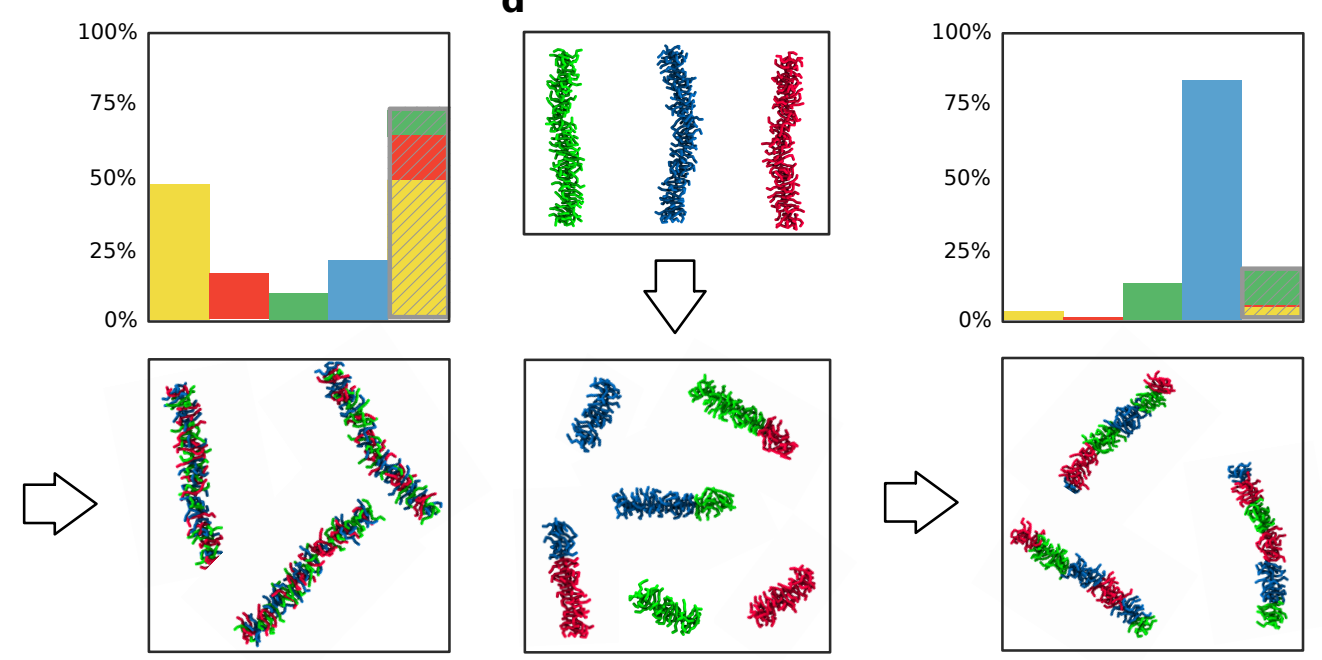

Figure 5. Equilibrium dynamics in supramolecular polymer systems. (a) Raw transition matrix (center) and transition probability sub-matrices (left and right, as in Figure 2a), comparing the $\mathbf{M}$ system with $\varepsilon=40 \mathrm{~kJ} \mathrm{~mol}^{-1}$ (top) and the BTA system at $T=340 \mathrm{~K}$ (bottom). (b) Same as (a), comparing the $\mathbf{M}$ system with $\varepsilon=50 \mathrm{~kJ} \mathrm{~mol}^{-1}$ (top) and the BTA system at $T=300 \mathrm{~K}$ (bottom). (c,d) Illustrative schemes of the mechanisms of inter-assembly communication. When the interaction between the self-assembling units is weaker (lower $\varepsilon$, or higher $T$ ), the fibres preferably communicate with each other exchanging monomers or relatively small fragments. When the interaction between self-assembling units is stronger (higher $\varepsilon$, or lower $T$ ), the inter-assembly communication proceeds mostly via large fibre fragmentation and coalescence. 
more chemically-relevant, flexible BTA model. In this sense, it is worth pointing out that the results discussed herein hold for supramolecular polymers composed of monomers that can be effectively modeled (on average) as globally solvophilic and directionally interacting units. In fact, when the monomers are more solvophobic, and non-directional interactions become non-negligible, the dynamics of the system might change. In such cases, it has been recently demonstrated that the exchange of monomers within the assemblies (monomer reshuffling) may become even more prominent than the exchange between fibres and their environment. ${ }^{44,48,57}$

The global and microscopic similarities and the analogous scaling behavior exhibited by the two different models (Figure 5 and Figure S27 in the SI) prove how even relatively simple self-assembling systems possess a rather complex dynamic character at the equilibrium. The obtained results demonstrate however that, not only the average assemblies sizes, but also the dynamic communication between aggregates that are formed at the supramolecular equilibrium is regulated by environmental variables (such as, e.g., temperature) as much as by molecular features of the self-assembling building blocks (e.g., monomer structure, interaction strength, etc.). This underlines the importance to move the attention from the structure of the fibres and from the monomers as individual entities, to the evaluation of the complexity that emerges in the system considered as a whole.

\section{Conclusions}

Supramolecular polymer systems are characterized by a dynamical equilibrium which confers to these materials an innate dynamic character and interesting bioinspired properties. The behavior of such complex systems is typically hard to understand, as their properties are controlled by molecular factors as much as by the collective dynamic behavior of the self-assembling entities that populate them. Studying the intricate network of collective interactions between the entities present in the systems with sufficient resolution to uncover the key molecular factors that control this complex dynamic behavior is a non-trivial but crucial challenge. Here we designed a molecular simulation approach which allows us to reach this goal. Employing coarse-grained models and molecular simulations of systems containing a large number of interacting monomers, we can study the structural and dynamical features of supramolecular polymer model examples at the thermodynamic equilibrium.

Our approach allows us to track the dynamic exchange events that occur in these systems. In this way, we can analyse in detail the molecular exchange processes that govern the dynamic equilibrium of supramolecular systems, unveiling details that cannot be captured with single-fibre simulations or stochastic models, studying supramolecular polymers on a purely average level. Our modeling approach provides insights on how do the assemblies communicate with each other, what are the exchange mechanisms, and how could it be possible, in principle, to control the collective behavior of these complex systems.

The results that we obtained change the usual way we look at supramolecular polymers from an average, macroscopic and structure-based, to a more dynamical, collective and microscopic point of view. The attention is moved to the dynamic behavior of the entities that statistically populate the system, and to the manner they dynamically and collectively communicate in what de facto is a complex molecular system. In such an exquisitely dynamical perspective, the supramolecular fibres that spontaneously form in the system are not the main subject, but they emerge statistically as a consequence of the dynamic behavior of monomeric entities that are continuously exchanged in-and-out the assemblies.

We believe that the approach presented herein can be applied to study a variety of supramolecular polymers as well as other types of dynamic self-assembling systems, and to explore routes to control the dynamical behavior of such complex systems by acting on the collective communication between their constitutive building blocks.

\section{Methods}

\section{Minimalistic M model}

The interacting hexagonal unit $\mathbf{M}$ is composed of seven beads, six shielding beads at the vertices of a regular hexagon and a central core bead, as depicted in Figure 1. The flat hexagonal geometry of the $\mathbf{M}$ monomer model is imposed by bonded interactions defined by harmonic bonds: the force constant between the nearest neighbor beads is $20000 \mathrm{~kJ} \mathrm{~mol}^{-1} \mathrm{~nm}^{-2}$ and the equilibrium length is $0.47 \mathrm{~nm}$. In order to keep the $\mathbf{M}$ hexagons planar, each shielding bead is also connected with the one at the opposite vertex of the hexagon by a harmonic bond with equilibrium length $0.94 \mathrm{~nm}$ and force constant of $15000 \mathrm{~kJ} \mathrm{~mol}^{-1} \mathrm{~nm}^{-2}$. The non-bonded interactions between beads are defined by Lennard-Jones (LJ) potentials, with constant $\sigma=0.47 \mathrm{~nm}$ and variable interaction strength $\varepsilon$. All the shielding beads interact weakly with each other and with the core beads $\left(\varepsilon=0.2 \mathrm{~kJ} \mathrm{~mol}^{-1}\right)$, while the interaction between core beads are stronger, with an interactions strength that varies across simulated systems $\left(40 \mathrm{~kJ} \mathrm{~mol}^{-1}, 45 \mathrm{~kJ} \mathrm{~mol}^{-1}\right.$ or $\left.50 \mathrm{~kJ} \mathrm{~mol}^{-1}\right)$. Such LJ parameters, plus the geometry of the $\mathbf{M}$ monomers, determine the tendency of the $\mathbf{M}$ monomers to interact directionally. All the model settings and parameters used herein are provided in the SI (in .GRO and .ITP GROMACS format).

\section{BTA model}

The minimalistic BTA model employed herein is the implicit-solvent version of the molecular model developed recently in Ref.48. The topology of the monomer is identical, while the bonded and non-bonded interaction parameters have been 
adapted to work consistently in the absence of explicit-solvent molecules . This model is representative of 3-fold symmetric monomers (having three solvophilic arms that surround a core) which interact directionally with each other in a good solvent (including, but not limited to, e.g., BTA monomers with alkyl side chains immersed in organic solvents, etc.). Initially built starting from the topology of fine-grained MARTINI-based CG BTA-C6 monomers,${ }^{37}$ the BTA model has a more abstract and general structure, optimized to obtain a behavior consistent with that of higher-resolution models. ${ }^{48}$ The BTA monomer is composed by 18 beads of three different types (see Figure 4a). At the center of the molecule there is a CG-bead (Figure 4a: in red), containing a rigid central dipole composed of two small, charged beads of $q= \pm 1.4 e$ (in yellow), oriented along the monomer axis. Three arms originate from the central bead, which are composed of 5 CG-beads, linearly connected to form a three-armed monomer. The intra-molecule bonded interactions are modelled via harmonic bond and angle potentials, the parameters are reported in the SI. The core and arm CG beads interact via Lennard-Jones (LJ) potential: the interactions between the beads of the side-chains have $\varepsilon=1 \mathrm{~kJ} \mathrm{~mol}^{-1}$ and $\sigma=0.47 \mathrm{~nm}$, the interactions between the side-chain and core beads have $\varepsilon=0.5 \mathrm{~kJ} \mathrm{~mol}^{-1}$ and $\sigma=0.47 \mathrm{~nm}$, while $\varepsilon=2.5 \mathrm{~kJ} \mathrm{~mol}^{-1}$ and $\sigma=0.47 \mathrm{~nm}$ for the core-core interactions. The charged beads that determine the central dipole have no LJ interactions, and interact with each other electrostatically, imparting directionality to the monomer-monomer interaction. Complete details on the models and force field parameters used for the BTA monomer model are provided in the SI (in .GRO and .ITP GROMACS format).

\section{CG-MD simulations}

The CG-MD simulations of the $\mathbf{M}$ model and of the BTA model were carried out using the GROMACS software ${ }^{61}$ (versions 2018.6 and 2020.2). All the MD simulations were performed in NVT conditions, using a constant volume for the simulation box of $22.056 \times 22.056 \times 22.056 \mathrm{~nm}^{3}$, with periodic boundary conditions, a constant number of molecules $N=500$, and a constant temperature. This corresponds to a monomer density of $0.0466 \mathrm{~nm}^{-3}$, equivalent to a concentration of $\sim 77 \mathrm{mM}$. The temperature was set to $T=300 \mathrm{~K}$, in the $\mathbf{M}$ model, and at different values (see main text) for the BTA model. The systems were simulated in implicit-solvent via Langevin dynamics, accounting for the friction of the solvent and thermal fluctuations of the system. We used the stochastic dynamics ( $\mathrm{sd}$ ) integrator, setting the inverse of the friction constant to $t a u-t=0.1 \mathrm{ps}$. tau-t also sets the coupling with the random force term, that determines the temperature of the system. The time step was set at $\Delta t=20 \mathrm{fs}$ for the $\mathbf{M}$ model and at $\Delta t=15 \mathrm{fs}$ for the BTA model simulations. The non-bonded interaction potentials were truncated and shifted at $r_{c}=1.1 \mathrm{~nm}$.

The equilibration phase of the $\mathbf{M}$ model was studied over six sets of simulations, $\varepsilon=40 \mathrm{~kJ} \mathrm{~mol}^{-1}$ (Figures $2 \mathrm{~d}, 2 \mathrm{e}, \mathrm{S} 2, \mathrm{~S} 8$ and S3) $\varepsilon=45 \mathrm{~kJ} \mathrm{~mol}^{-1}$ (Figures S4, S5, S9) and $\varepsilon=50 \mathrm{~kJ} \mathrm{~mol}^{-1}$ (Figures S6, S7, S10) starting from either random (R) or stacked (S) configurations - i.e., starting from $N=500$ randomly distributed monomers (R), or arranged in 20 pre-stacked fibres composed of 25 monomers each (S). Each of these simulations are $20 \mu$ s long and sampled every ns.

The analysis at the equilibrium of the $\mathbf{M}$ model has been performed for three different simulations, with $\varepsilon=40 \mathrm{~kJ} \mathrm{~mol}^{-1}$, $\varepsilon=45 \mathrm{~kJ} \mathrm{~mol}^{-1}$ and $\varepsilon=50 \mathrm{~kJ} \mathrm{~mol}^{-1}$ (Figures $2 \mathrm{c}$ and 2f, 3, S12, S15). For each system we performed a total of $40 \mu \mathrm{s}$ of CG-MD, starting from randomly dispersed monomers. The first $20 \mu \mathrm{s}$ of CG-MD are considered as an equilibration stage (corresponding to the R simulations mentioned before), while the trajectory 20 to $40 \mu$ s is employed for the equilibrium studies. During this second part of the CG-MD we sampled the conformations every $300 \mathrm{ps}$.

For the BTA model, we performed three sets of simulations at different temperatures: $T=340 \mathrm{~K}, T=320 \mathrm{~K}$ and $T=300 \mathrm{~K}$ (Figures 4, 5, S17, S24, S25, S27). As in the M model, for each system we performed a total of $40 \mu$ s of CG-MD, starting from randomly dispersed monomers. The first $20 \mu$ s of CG-MD (sampled every $750 \mathrm{ps}$, see Figure S16) contain the equilibration phase, while the CG-MD from 20 to $40 \mu$ s is employed for the study in equilibrium conditions (sampling every $300 \mathrm{ps}$ ).

\section{Analysis of the CG-MD simulations}

All the analysis were carried out by means of python scripts employing the MD-Analysis package ${ }^{62,63}$, with the exception of the analysis reported in Figures 2c, 4d, S8, S9, S10 and the analysis reported in Figure S1, which were performed using the clustsize and $r d f$ GROMACS tools, respectively. The snapshots in Figures 3c, 2a-b and S11 were rendered with VMD. ${ }^{64}$

For the analyses of monomer aggregation, we proceeded as follows: two $\mathbf{M}$ monomers were considered in contact (i.e., they belonged to the same assembly) if their core beads lied within a distance of $r_{\text {cut }}=0.6 \mathrm{~nm}$; we chose this value for $r_{\text {cut }}$ as this radius includes the first peak of the radial distribution function $(g(r))$ (Figure S1). For the BTA model, the contact radius $r_{\text {cut }}$ has been fixed at $0.6 \mathrm{~nm}$, obtained following the same criterion. We also tested how a different choice of the cutoff can impact the analysis (vide infra).

The molecular traffic and flux analyses ${ }^{54}$, monitoring the dynamic behavior of the system, were performed both for equilibration (the first $20 \mu \mathrm{s}$ ) part and for the equilibrium part of the trajectories (from 20 to $40 \mu \mathrm{s}$, see Figures $2 \mathrm{~d}$ bottom, $2 \mathrm{f}$ and $4 \mathrm{e}$ ). Both quantities are cumulative, i.e., they keep track of the previous behavior of the system and are defined as:

$$
T(\tau)=\sum_{t=0}^{\tau} \frac{b(t)+u(t)}{N} \quad F(\tau)=\sum_{t=0}^{\tau} \frac{b(t)-u(t)}{N},
$$


where $N=500$ is the number of monomers in the system. The traffic $(T)$ indicates how many dynamical events (binding $b$ and unbinding $u$ ) per-monomer have occurred until $t=\tau$, while the flux $(F)$ indicates the balance (difference) between such events.

The transition matrices reported in Figures $3 \mathrm{a}$ and 5, record the binding/unbinding events involving each monomer, during the equilibrium phases of CG-MD, i.e., from $20 \mu \mathrm{s}$ to $40 \mu \mathrm{s}$, with a sampling step of $\Delta \tau=300 \mathrm{ps}$, for both the M and BTA models. The raw-data transition matrices (central panels in Figs.3a, 5a and b) in report the number of monomer transitions from an assembly of size $i$ (row index) to an assembly of size $j$ (column index), colored by a logarithmic scale. Since the system is at the equilibrium, and detailed balance is met, the matrices are symmetric, and the frequency of binding events (upper triangular region of the matrix) equates the frequency of the corresponding unbinding events (lower triangular). We also reported the rate

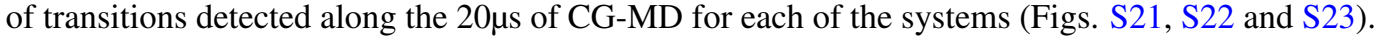

The transition probability matrices (partially reported in the left and right panels of Figs.3a, 5a and b), are normalized over each row so that they indicate the percentage probability that a monomer from a construct of given size-range $i$ transits to a construct of size-range $j$ during the sampling step $\Delta \tau$.

To classify the different mechanisms of exchange between the self-assembled fibres we divided the entries of the row transition matrices by their row or column index, depending if they were part of the superior or inferior triangular matrix respectively. In this way we obtained assembly transition matrices, which list the number of transitions of an aggregate of size $i$ into an aggregate of size $j$ (Figs.3e:left and S25:left column). This holds under the assumption of binary events, i.e., the transitions collected in the matrices involve only two assemblies at once. This condition is not strictly satisfied, but we verified that the qualitative message of the outcome is robust in this sense (by computing the transition matrices with a different sampling step $\Delta \tau$ and contact radius $r_{\text {cut }}$, see below).

We facilitated the interpretation of assembly transition matrices via the illustrative scheme/legend of Figure $3 \mathrm{~d}$ : the matrix is divided into four areas, which identify the class of transition events based on two characteristic aggregate sizes, $A=\langle$ size $\rangle$ and $E=A / 5$, related to the typical size of the formed aggregates, as explained in the main text. We then computed the probability of each class of events by summing all the events included in each region, and dividing this number by the total number of transition events recorded in the matrix (i.e., the sum of all the entries except the diagonal). This provided the probabilities reported in the main text and the matrices depicted in Figures 3e, S15 and S25.

To further investigate the dependence of the results changing the spatial and temporal resolution, we computed the statistical weight of different polymerization/depolymerization mechanisms (the percentage associated to the areas defined in Figure $3 \mathrm{~d}$ ) by changing the contact cutoff radius $r_{\text {cut }}$ and the sampling step $\Delta \tau$. Firstly, we analysed the transitions detected by using $r_{\text {cut }}=0.7 \mathrm{~nm}$. We obtained the results reported in Figure S26, where the probabilities show a similar trend to that obtained with the $0.6 \mathrm{~nm}$ cutoff radius used in the rest of the analyses (Figure S25), for both the $\mathbf{M}$ and the BTA systems. Then, changing the temporal resolution of the sampling, i.e. increasing the sampling step to $\Delta \tau=3 \mathrm{~ns}$, we computed the transition matrices for both the $\mathbf{M}$ and the BTA systems (see Figure S28, S29) and the probabilities associated to the different areas of the assembly transition matrices, obtaining the results reported in Figure S30. Also these additional analyses show a trend similar to the original ones for both the $\mathbf{M}$ and the BTA systems, demonstrating the robustness of our conclusions.

\section{References}

1. Aida, T., Meijer, E. \& Stupp, S. I. Functional supramolecular polymers. Science 335, 813-817 (2012).

2. van der Zwaag, D., de Greef, T. F. \& Meijer, E. W. Programmable supramolecular polymerizations. Angewandte Chemie Int. Ed. 54, 8334-8336 (2015).

3. Webber, M. J., Appel, E. A., Meijer, E. W. \& Langer, R. Supramolecular biomaterials. Nat. Mater 15, 13-26, 10.1038/ nmat4474 (2016).

4. Savyasachi, A. J. et al. Supramolecular Chemistry: A Toolkit for Soft Functional Materials and Organic Particles. Chem 3, 764-811, 10.1016/j.chempr.2017.10.006 (2017).

5. Brunsveld, L., Folmer, B. J. B., Meijer, E. W. \& Sijbesma, R. P. Supramolecular Polymers. Chem. Rev. 101, 4071-4098, 10.1021/cr990125q (2001).

6. Lehn, J. M. Dynamers: Dynamic molecular and supramolecular polymers. Prog. Polym. Sci. 30, 814-831, 10.1016/j. progpolymsci.2005.06.002 (2005).

7. Davis, A. V., Yeh, R. M. \& Raymond, K. N. Supramolecular assembly dynamics. Proc. Natl. Acad. Sci. United States Am. 99, 4793-4796, 10.1073/pnas.052018299 (2002).

8. Yan, X., Wang, F., Zheng, B. \& Huang, F. Stimuli-responsive supramolecular polymeric materials. Chem. Soc. Rev. 41, 6042-6065, 10.1039/C2CS35091B (2012).

9. Merindol, R. \& Walther, A. Materials learning from life: Concepts for active, adaptive and autonomous molecular systems. Chem. Soc. Rev. 46, 5588-5619, 10.1039/C6CS00738D (2017). 
10. Messmore, B. W., Hulvat, J. F., Sone, E. D. \& Stupp, S. I. Synthesis, Self-Assembly, and Characterization of Supramolecular Polymers from Electroactive Dendron Rodcoil Molecules. J. Am. Chem. Soc. 126, 14452-14458, 10.1021/ja049325w (2004).

11. Liu, Y., Wang, Z. \& Zhang, X. Characterization of supramolecular polymers. Chem. Soc. Rev. 41, 5922-5932, 10.1039/ C2CS35084J (2012).

12. Casellas, N. M. et al. From isodesmic to highly cooperative: reverting the supramolecular polymerization mechanism in water by fine monomer design. Chem. Commun. 54, 4112-4115, 10.1039/C8CC01259H (2018).

13. Mishra, A. et al. Biomimetic temporal self-assembly via fuel-driven controlled supramolecular polymerization. Nat. Commun. 9, 1295, 10.1038/s41467-018-03542-z (2018).

14. Aliprandi, A., Mauro, M. \& De Cola, L. Controlling and imaging biomimetic self-assembly. Nat. Chem. 8, 10-15, 10.1038/nchem.2383 (2016).

15. Albertazzi, L. et al. Spatiotemporal control and superselectivity in supramolecular polymers using multivalency. Proc. Natl. Acad. Sci. United States Am. 110, 12203-12208, 10.1073/pnas.1303109110 (2013).

16. Baker, M. B. et al. Consequences of chirality on the dynamics of a water-soluble supramolecular polymer. Nat. Commun. 6, 6234, 10.1038/ncomms7234 (2015).

17. Albertazzi, L. et al. Probing exchange pathways in one-dimensional aggregates with super-resolution microscopy. Science 344, 491-495, 10.1126/science.1250945 (2014).

18. Da Silva, R. M. et al. Super-resolution microscopy reveals structural diversity in molecular exchange among peptide amphiphile nanofibres. Nat. Commun. 7, 11561, 10.1038/ncomms11561 (2016).

19. Lou, X. et al. Dynamic diversity of synthetic supramolecular polymers in water as revealed by hydrogen/deuterium exchange. Nat. Commun. 8, 15420, 10.1038/ncomms15420 (2017).

20. Schoenmakers, S. M. C. et al. Impact of the water-compatible periphery on the dynamic and structural properties of benzene-1,3,5-tricarboxamide based amphiphiles. Chem. Сотmun. 54, 11128-11131, 10.1039/C8CC04818E (2018).

21. Sarkar, A. et al. Self-Sorted, Random, and Block Supramolecular Copolymers via Sequence Controlled, Multicomponent Self-Assembly. J. Am. Chem. Soc. 142, 7606-7617, 10.1021/jacs.0c01822 (2020).

22. Moreno-Alcántar, G. et al. Solvent-Driven Supramolecular Wrapping of Self-Assembled Structures. Angewandte Chemie Int. Ed. 60, 5407-5413, 10.1002/anie.202013474 (2021).

23. Oosawa, F. \& Kasai, M. A theory of linear and helical aggregations of macromolecules. J. Mol. Biol. 4, 10-21, 10.1016/S0022-2836(62)80112-0 (1962).

24. Knowles, T. P. J. et al. An Analytical Solution to the Kinetics of Breakable Filament Assembly. Science 326, 1533-1537, 10.1126/science.1178250 (2009).

25. Markvoort, A. J., Ten Eikelder, H. M., Hilbers, P. A., De Greef, T. F. \& Meijer, E. W. Theoretical models of nonlinear effects in two-component cooperative supramolecular copolymerizations. Nat. Commun. 2, 509, 10.1038/ncomms 1517 (2011).

26. Korevaar, P. A. et al. Pathway complexity in supramolecular polymerization. Nature 481, 492-496, 10.1038/nature10720 (2012).

27. Markvoort, A. J., Ten Eikelder, H. M., Hilbers, P. A. \& De Greef, T. F. Fragmentation and coagulation in supramolecular (Co)polymerization kinetics. ACS Cent. Sci. 2, 232-241, 10.1021/acscentsci.6b00009 (2016).

28. Michaels, T. C. et al. Chemical Kinetics for Bridging Molecular Mechanisms and Macroscopic Measurements of Amyloid Fibril Formation. Annu. Rev. Phys. Chem. 69, 273-298, 10.1146/annurev-physchem-050317-021322 (2018).

29. Ten Eikelder, H. M. \& Markvoort, A. J. Mass-Balance Models for Scrutinizing Supramolecular (Co)polymerizations in Thermodynamic Equilibrium. Accounts Chem. Res. 52, 3465-3474, 10.1021/acs.accounts.9b00487 (2019).

30. Kumar, M. et al. A dynamic supramolecular polymer with stimuli-responsive handedness for in situ probing of enzymatic ATP hydrolysis. Nat. communications 5, 5793 (2014).

31. Garzoni, M. et al. Effect of H-Bonding on Order Amplification in the Growth of a Supramolecular Polymer in Water. $J$. Am. Chem. Soc. 138, 13985-13995, 10.1021/jacs.6b07530 (2016).

32. Bejagam, K. K., Fiorin, G., Klein, M. L. \& Balasubramanian, S. Supramolecular polymerization of benzene-1,3,5tricarboxamide: A molecular dynamics simulation study. J. Phys. Chem. B 118, 5218-5228, 10.1021/jp502779z (2014). 
33. Tantakitti, F. et al. Energy landscapes and functions of supramolecular systems. Nat. Mater. 15, 469-476, 10.1038/nmat4538 (2016).

34. Shyshov, O. et al. Living supramolecular polymerization of fluorinated cyclohexanes. Nat Commun 12, 3134, 10.1038/ s41467-021-23370-y (2021).

35. Lee, O.-S., Cho, V. \& Schatz, G. C. Modeling the Self-Assembly of Peptide Amphiphiles into Fibers Using Coarse-Grained Molecular Dynamics. Nano Lett. 12, 4907-4913, 10.1021/n1302487m (2012).

36. Bejagam, K. K. \& Balasubramanian, S. Supramolecular Polymerization: A Coarse Grained Molecular Dynamics Study. The J. Phys. Chem. B 119, 5738-5746, 10.1021/acs.jpcb.5b01655 (2015).

37. Bochicchio, D. \& Pavan, G. M. From Cooperative Self-Assembly to Water-Soluble Supramolecular Polymers Using Coarse-Grained Simulations. ACS Nano 11, 1000-1011, 10.1021/acsnano.6b07628 (2017).

38. Bochicchio, D. \& Pavan, G. M. Effect of Concentration on the Supramolecular Polymerization Mechanism via ImplicitSolvent Coarse-Grained Simulations of Water-Soluble 1,3,5-Benzenetricarboxamide. J. Phys. Chem. Lett. 8, 3813-3819, 10.1021/acs.jpclett.7b01649 (2017).

39. Šarić, A., Chebaro, Y. C., Knowles, T. P. J. \& Frenkel, D. Crucial role of nonspecific interactions in amyloid nucleation. PNAS 111, 17869-17874, 10.1073/pnas.1410159111 (2014).

40. Marenda, M., Orlandini, E. \& Micheletti, C. Discovering privileged topologies of molecular knots with self-assembling models. Nat. Commun. 9, 3051, 10.1038/s41467-018-05413-z (2018).

41. Zumbro, E., Witten, J. \& Alexander-Katz, A. Computational Insights into Avidity of Polymeric Multivalent Binders. Biophys. J. 117, 892-902, 10.1016/j.bpj.2019.07.026 (2019).

42. Chakraborty, S., Berac, C. M., Kemper, B., Besenius, P. \& Speck, T. Modeling Supramolecular Polymerization: The Role of Steric Effects and Hydrophobic Interactions. Macromolecules 52, 7661-7667, 10.1021/acs.macromol.9b01435 (2019).

43. Perego, C., Pesce, L., Capelli, R., George, S. J. \& Pavan, G. M. Multiscale Molecular Modelling of ATP-Fueled Supramolecular Polymerisation and Depolymerisation**. ChemSystemsChem 3, 1-10, 10.1002/syst.202000038 (2021).

44. Bochicchio, D., Salvalaglio, M. \& Pavan, G. M. Into the dynamics of a supramolecular polymer at submolecular resolution. Nat. Commun. 8, 147, 10.1038/s41467-017-00189-0 (2017).

45. Jung, S. H., Bochicchio, D., Pavan, G. M., Takeuchi, M. \& Sugiyasu, K. A Block Supramolecular Polymer and Its Kinetically Enhanced Stability. J. Am. Chem. Soc. 140, 10570-10577, 10.1021/jacs.8b06016 (2018).

46. Bochicchio, D. \& Pavan, G. M. Molecular modelling of supramolecular polymers. Adv. Physics: X 3, 1436408, 10.1080/23746149.2018.1436408 (2018).

47. Alessandri, R., Grünewald, F. \& Marrink, S. J. The Martini Model in Materials Science. Adv. Mater. 33, 2008635, 10.1002/adma.202008635 (2021).

48. de Marco, A. L., Bochicchio, D., Gardin, A., Doni, G. \& Pavan, G. M. Controlling Exchange Pathways in Dynamic Supramolecular Polymers by Controlling Defects. ACS Nano 15, 14229-14241, 10.1021/acsnano.1c01398 (2021).

49. Torchi, A., Bochicchio, D. \& Pavan, G. M. How the dynamics of a supramolecular polymer determines its dynamic adaptivity and stimuli-responsiveness: Structure-dynamics-property relationships from coarse-grained simulations. The J. Phys. Chem. B 122, 4169-4178, 10.1021/acs.jpcb.8b00428 (2018).

50. Lionello, C. et al. Toward Chemotactic Supramolecular Nanoparticles: From Autonomous Surface Motion Following Specific Chemical Gradients to Multivalency-Controlled Disassembly. ACS Nano 15, 16149-16161, 10.1021/acsnano. $1 \mathrm{c} 05000$ (2021).

51. Bianchi, E. Patchy Colloids: A Theoretical and Numerical Perspective on Functionalized Units for Self-Assembly, vol. 13 (Elsevier Ltd. (Oxford), 2019), 1 edn.

52. Prestipino, S., Gazzillo, D., Munaò, G. \& Costa, D. Complex Self-Assembly from Simple Interaction Rules in Model Colloidal Mixtures. J. Phys. Chem. B 123, 9272-9280, 10.1021/acs.jpcb.9b08617 (2019). 2002.05930.

53. Geng, Y., van Anders, G., Dodd, P. M., Dshemuchadse, J. \& Glotzer, S. C. Engineering entropy for the inverse design of colloidal crystals from hard shapes. Sci. Adv. 5, eaaw0514, 10.1126/sciadv.aaw0514 (2019). https://www.science.org/doi/ pdf/10.1126/sciadv.aaw0514.

54. Grant, J., Jack, R. L. \& Whitelam, S. Analyzing mechanisms and microscopic reversibility of self-assembly. The J. Chem. Phys. 135, 214505-214515, 10.1063/1.3662140 (2011). 
55. Leenders, C. M. A. et al. Supramolecular polymerisation in water; elucidating the role of hydrophobic and hydrogen-bond interactions. Soft Matter 12, 2887-2893, 10.1039/C5SM02843D (2016).

56. Leenders, C. M. A. et al. Supramolecular polymerization in water harnessing both hydrophobic effects and hydrogen bond formation. Chem. Commun. 49, 1963-1965, 10.1039/C3CC38949A (2013).

57. Gasparotto, P., Bochicchio, D., Ceriotti, M. \& Pavan, G. M. Identifying and Tracking Defects in Dynamic Supramolecular Polymers. The J. Phys. Chem. B 124, 589-599, 10.1021/acs.jpcb.9b11015 (2020).

58. Zhao, D. \& Moore, J. S. Nucleation-elongation: A mechanism for cooperative supramolecular polymerization. Org. \& Biomol. Chem. 1, 3471-3491, 10.1039/B308788C (2003).

59. Smulders, M. M. J., Schenning, A. P. H. J. \& Meijer, E. W. Insight into the Mechanisms of Cooperative Self-Assembly: The "Sergeants-and-Soldiers" Principle of Chiral and Achiral C3-Symmetrical Discotic Triamides. J. Am. Chem. Soc. 130, 606-611, 10.1021/ja075987k (2008).

60. Smulders, M. M. J. et al. How to Distinguish Isodesmic from Cooperative Supramolecular Polymerisation. Chem. - A Eur. J. 16, 362-367, 10.1002/chem.200902415 (2010).

61. Hess, B., Kutzner, C., van der Spoel, D. \& Lindahl, E. GROMACS 4: Algorithms for Highly Efficient, Load-Balanced, and Scalable Molecular Simulation. J. Chem. Theory Comput. 4, 435-447, 10.1021/ct700301q (2008).

62. Gowers, R. J. et al. MDAnalysis: A Python Package for the Rapid Analysis of Molecular Dynamics Simulations. In Benthall, S. \& Rostrup, S. (eds.) Proceedings of the 15th Python in Science Conference, 98-105, 10.25080/Majora-629e541a-00e (2016).

63. Michaud-Agrawal, N., Denning, E. J., Woolf, T. B. \& Beckstein, O. MDAnalysis: A toolkit for the analysis of molecular dynamics simulations. J. Comput. Chem. 32, 2319-2327, 10.1002/jcc.21787 (2011).

64. Humphrey, W., Dalke, A. \& Schulten, K. VMD - Visual Molecular Dynamics. J. Mol. Graph. 14, 33-38 (1996).

\section{Data availability}

Details on the procedures for the parametrization of the molecular models and on the simulations' setup, along with additional simulation data, are provided in the Methods section and in the Supplementary Information file. Complete data and materials pertaining to the molecular simulations conducted herein (input files, model files, raw data, analysis tools, etc.) are available at: https://github.com/GMPavanLab/ComplexSPs (this link will be replaced with a definitive Zenodo link upon acceptance of the final version of this paper). Other information needed is available from the corresponding author upon reasonable request.

\section{Acknowledgements}

G.M.P. acknowledges the funding received by the ERC under the European Union's Horizon 2020 research and innovation program (grant agreement no. 818776 - DYNAPOL) and by the Swiss National Science Foundation (SNSF grants IZLIZ2_183336). The authors also acknowledge the computational resources provided by the Swiss National Supercomputing Center (CSCS), by CINECA and by HPC@POLITO (http://www.hpc.polito.it).

\section{Author contributions}

M.C., C.P. and A.d.M. developed the molecular models. M.C. performed the simulations. M.C., C.P. and G.M.P. analysed the results. G.M.P. conceived the research and supervised the work. M.C., C.P. and G.M.P wrote the manuscript.

\section{Competing interests statement}

The authors declare no competing interests. 


\title{
Supporting Information for: Molecular Communications in Complex Systems of Dynamic Supramolecular Polymers
}

\author{
Martina Crippa $^{1}$, Claudio Perego ${ }^{2}$, Anna L. de Marco ${ }^{2,3}$, and Giovanni M. Pavan ${ }^{1,2, *}$ \\ ${ }^{1}$ Department of Applied Science and Technology, Politecnico di Torino, Corso Duca degli Abruzzi 24, 10129 Torino, \\ Italy \\ ${ }^{2}$ Department of Innovative Technologies, University of Applied Sciences and Arts of Southern Switzerland, Polo \\ Universitario Lugano, Campus Est, Via la Santa 1, 6962 Lugano-Viganello, Switzerland \\ ${ }^{3}$ Department of Physics, Università degli Studi di Genova, Via Dodecaneso 33, 16100 Genova, Italy \\ *corresponding author: Giovanni M. Pavan (giovanni.pavan@polito.it)
}




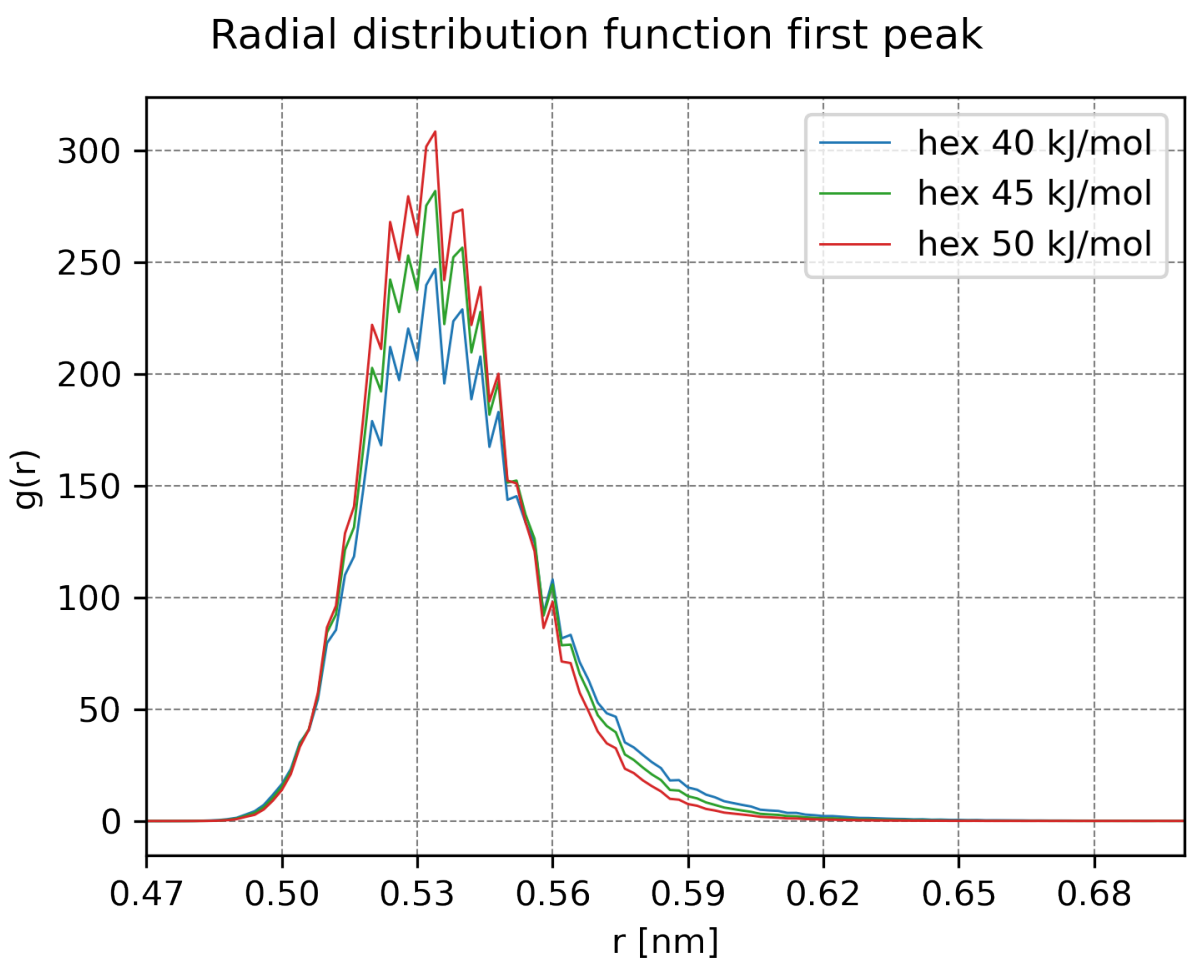

Figure S1. First peak of the radial distribution function for the equilibrium part of three different $\mathbf{M}$ systems $\left(\varepsilon=40 \mathrm{~kJ} \mathrm{~mol}^{-1}, 45, \mathrm{~kJ} \mathrm{~mol}^{-1}\right.$ and $\left.50 \mathrm{~kJ} \mathrm{~mol}^{-1}\right)$. 

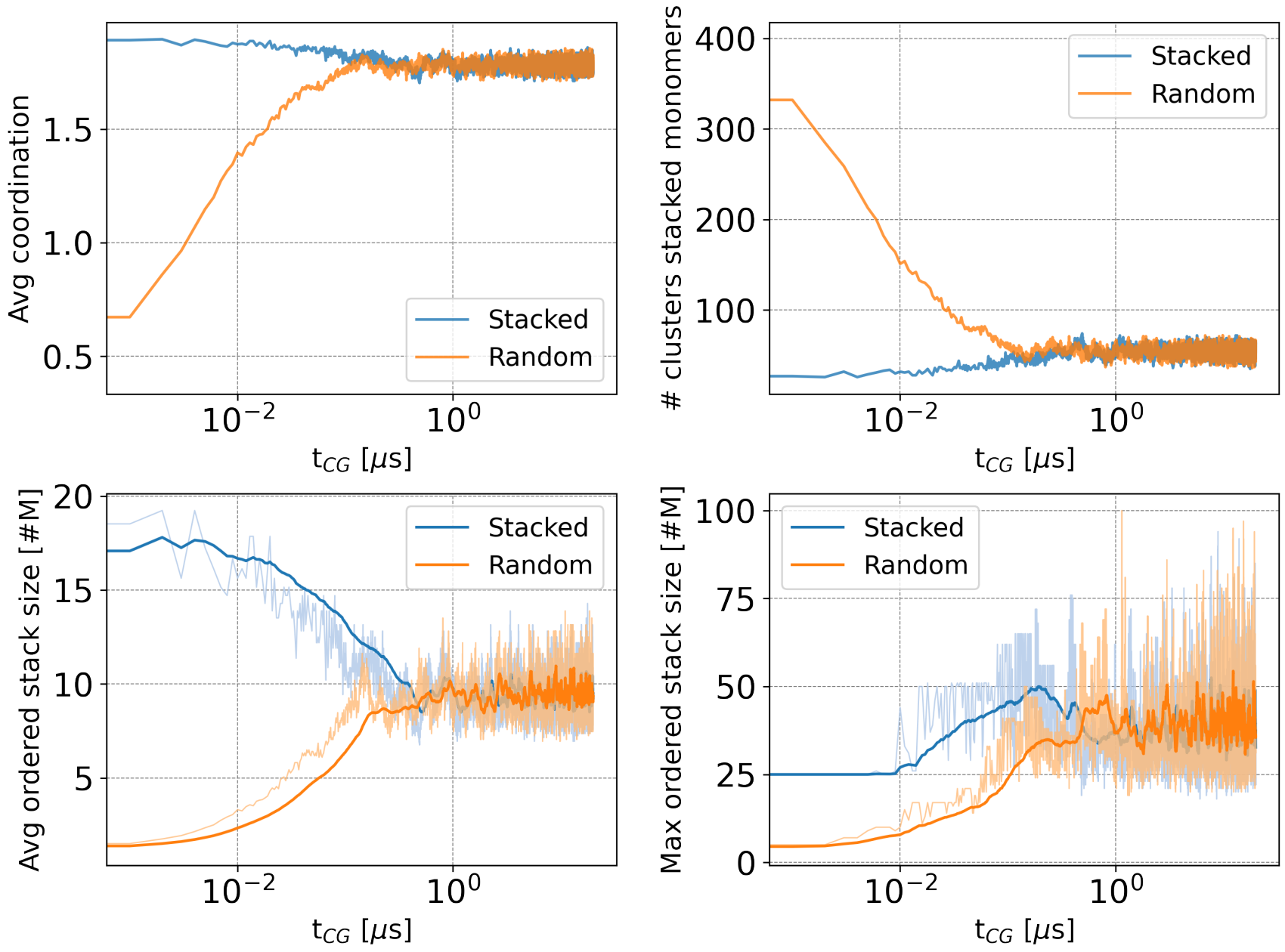

Figure S2. Self-assembly observables for the $\mathrm{R}$ and $\mathrm{S}$ systems interacting with $\varepsilon=40 \mathrm{~kJ} \mathrm{~mol}^{-1}$ : average coordination (top left), number of assemblies (top right), average size of assemblies (bottom left), size of the largest assembly (bottom right).
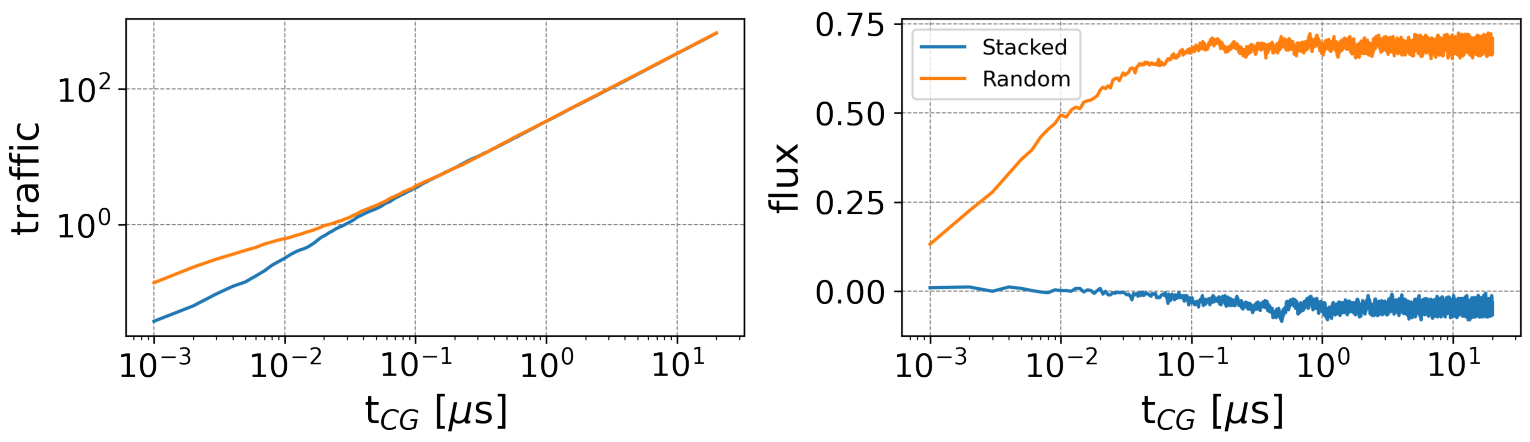

Figure S3. Traffic and flux for the R and S systems interacting with $\varepsilon=40 \mathrm{~kJ} \mathrm{~mol}^{-1}$. 

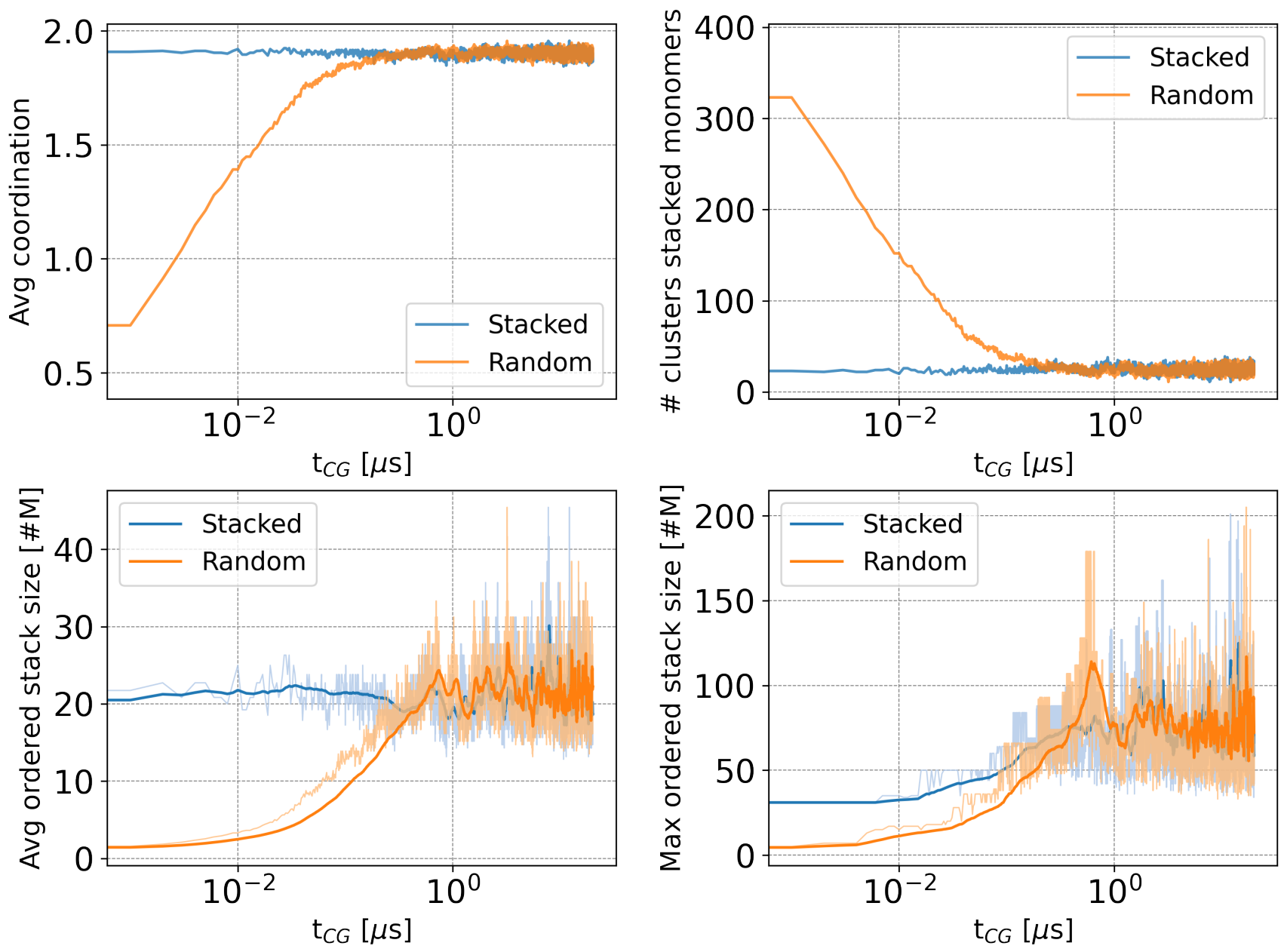

Figure S4. Self-assembly observables for the $\mathrm{R}$ and $\mathrm{S}$ systems interacting with $\varepsilon=45 \mathrm{~kJ} \mathrm{~mol}^{-1}$ : average coordination (top left), number of assemblies (top right), average size of assemblies (bottom left), size of the largest assembly (bottom right).
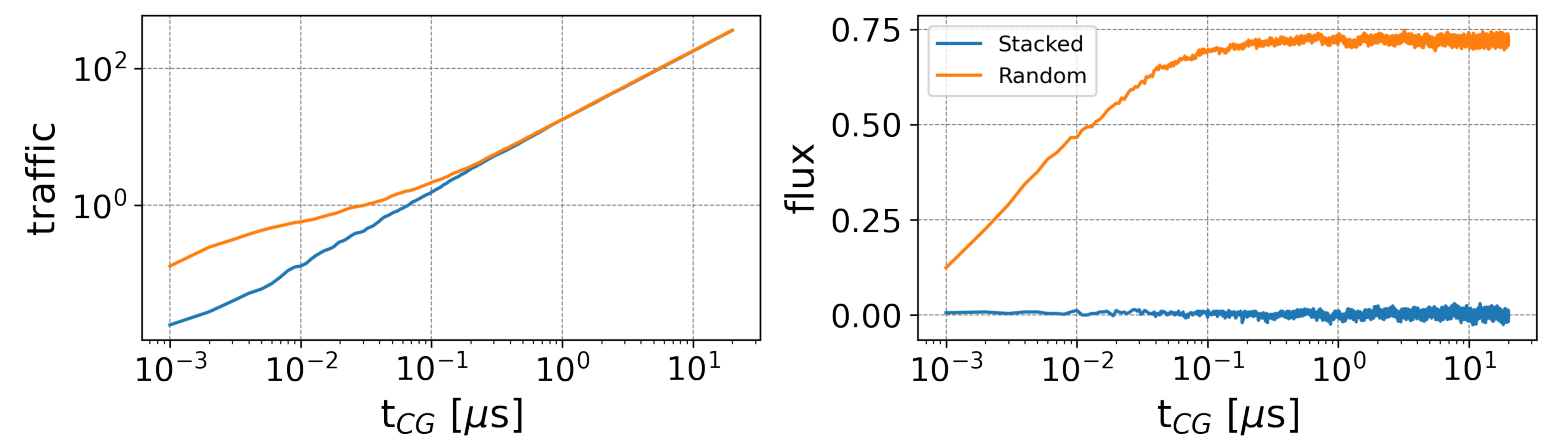

Figure S5. Traffic and flux for the R and S systems interacting with $\varepsilon=45 \mathrm{~kJ} \mathrm{~mol}^{-1}$. 

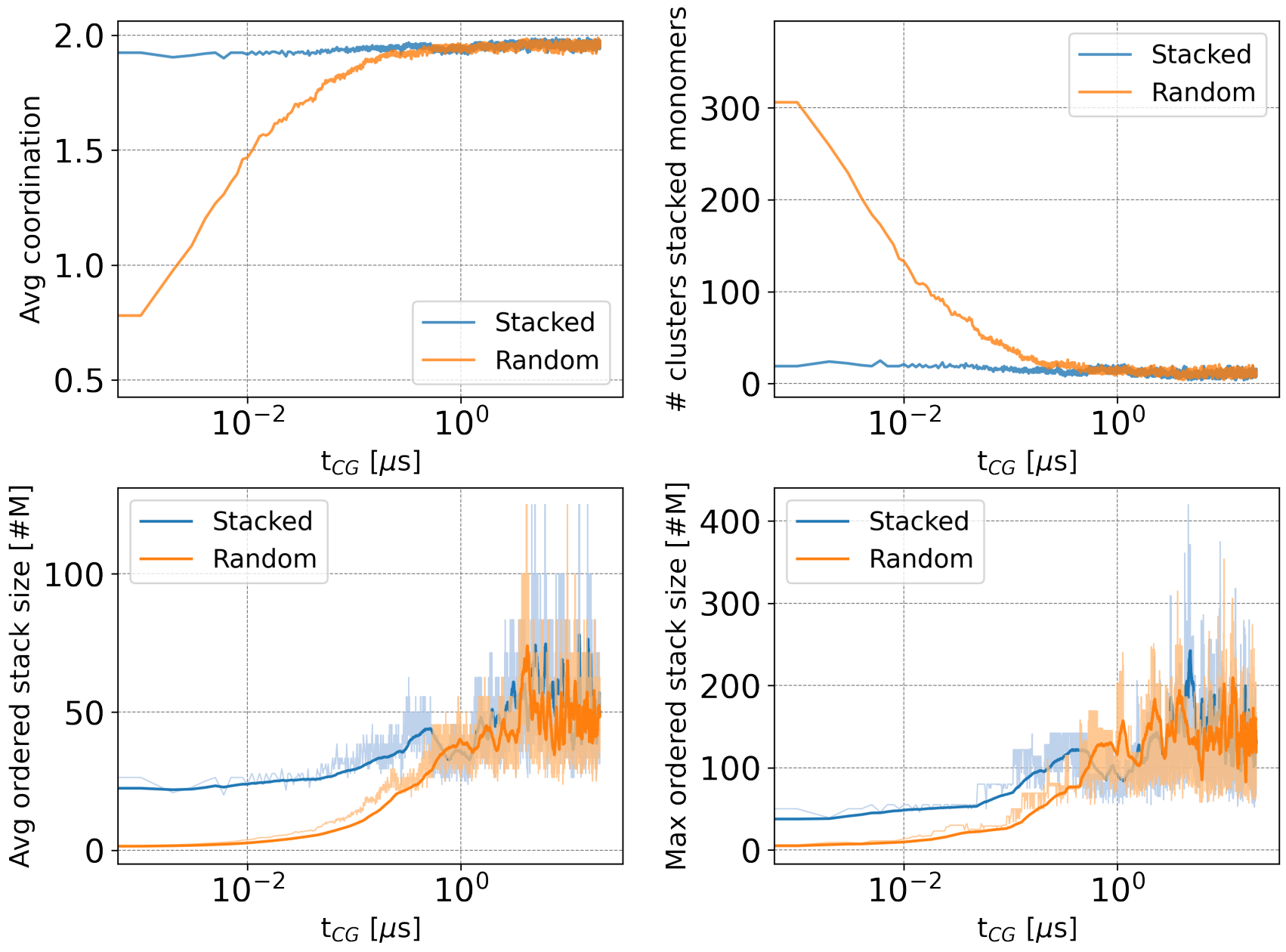

Figure S6. Self-assembly observables for the $\mathrm{R}$ and $\mathrm{S}$ systems interacting with $\varepsilon=50 \mathrm{~kJ} \mathrm{~mol}^{-1}$ : average coordination (top left), number of assemblies (top right), average size of assemblies (bottom left), size of the largest assembly (bottom right).
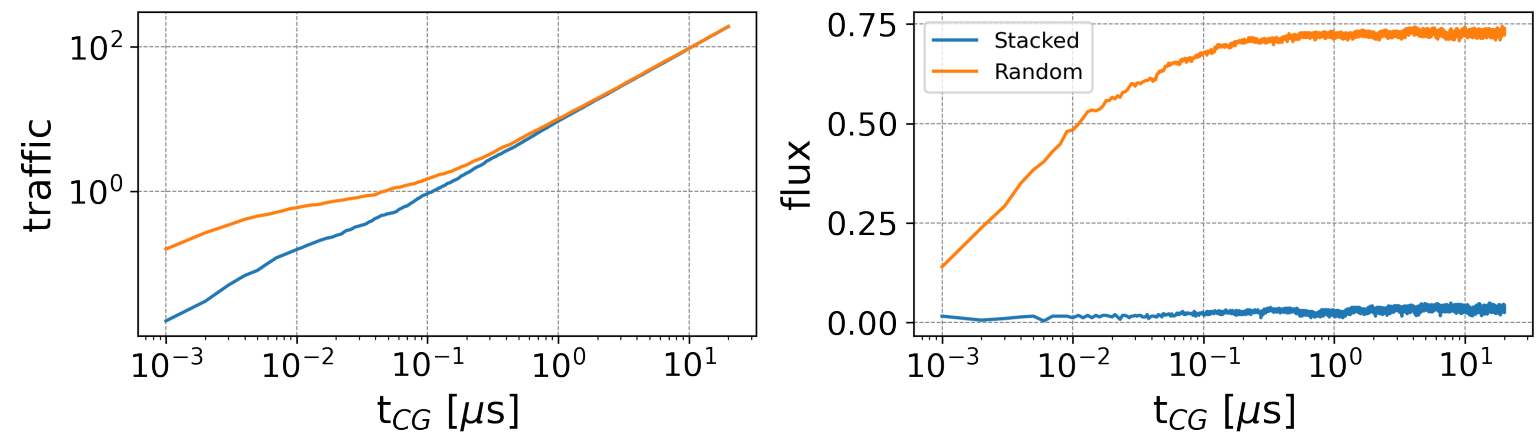

Figure S7. Traffic and flux for the R and S systems interacting with $\varepsilon=50 \mathrm{~kJ} \mathrm{~mol}^{-1}$. 

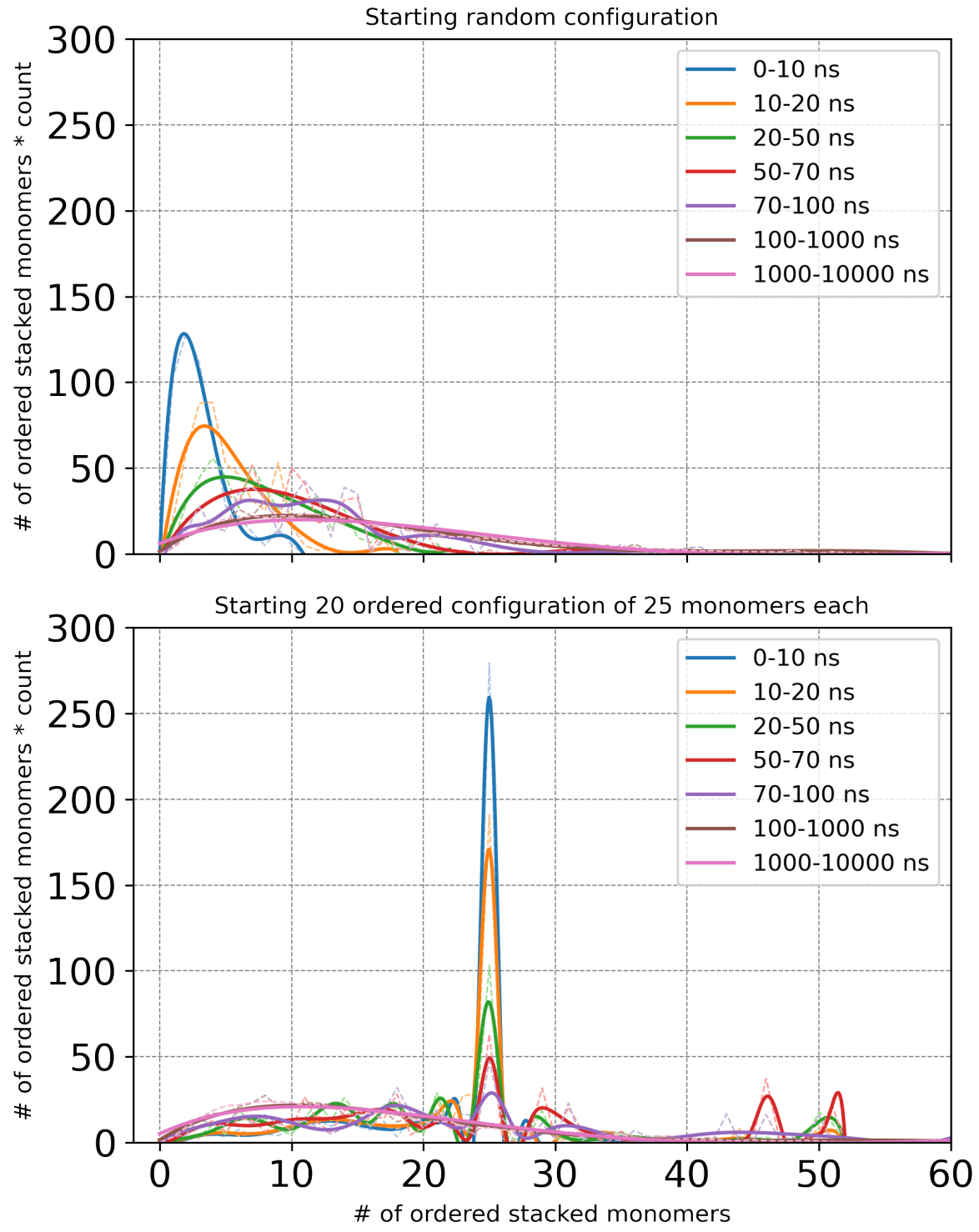

Figure S8. Distribution of monomers into aggregates of different sizes computed over different time intervals of the CG-MD trajectory, comparing $\mathrm{R}$ (top) and $\mathrm{S}$ (bottom) systems interacting by $\varepsilon=40 \mathrm{~kJ} \mathrm{~mol}^{-1}$. Comparing the two panels, convergence between the $\mathrm{S}$ and $\mathrm{R}$ system population distributions after $t_{1}=1 \mu \mathrm{s}$ (pink curve) is observed. 

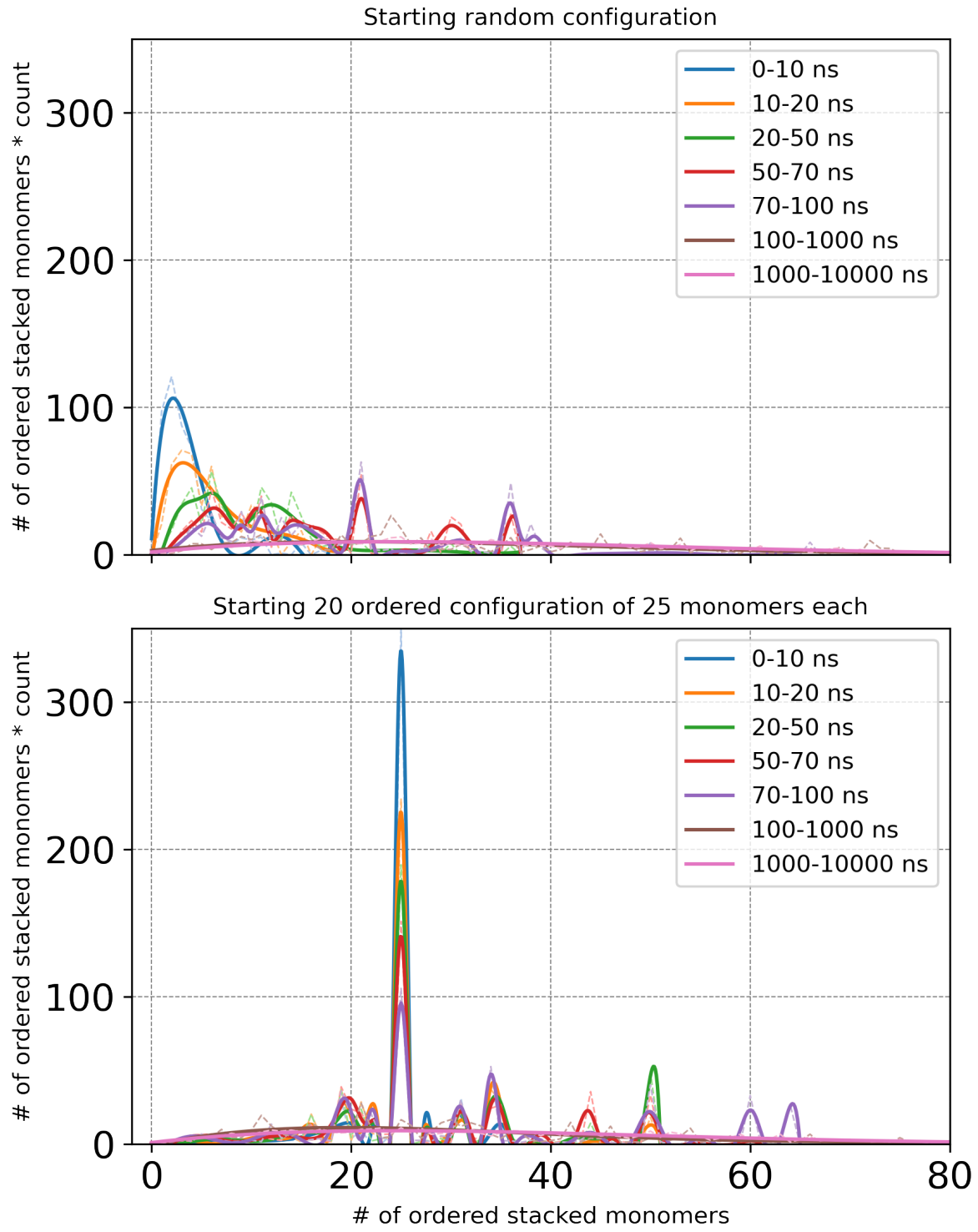

Figure S9. Distribution of monomers into aggregates of different sizes computed over different time intervals of the CG-MD trajectory, comparing $\mathrm{R}$ (top) and $\mathrm{S}$ (bottom) systems interacting by $\varepsilon=45 \mathrm{~kJ} \mathrm{~mol}^{-1}$. Comparing the two panels, convergence between the $\mathrm{S}$ and $\mathrm{R}$ system population distributions after $t_{1}=1 \mu \mathrm{s}$ (pink curve) is observed. 

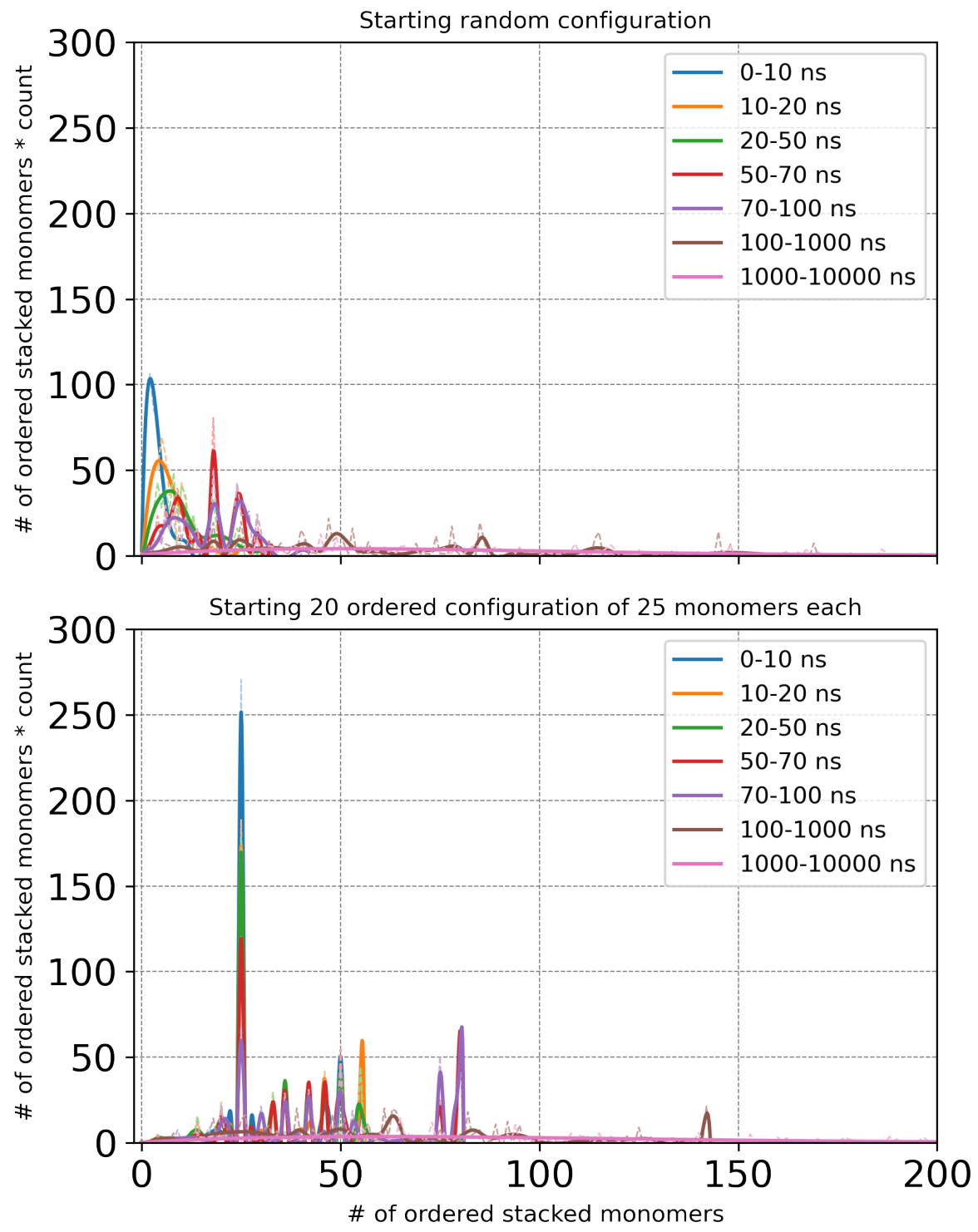

Figure S10. Distribution of monomers into aggregates of different sizes computed over different time intervals of the CG-MD trajectory, comparing R (top) and $\mathrm{S}$ (bottom) systems interacting by $\varepsilon=50 \mathrm{~kJ} \mathrm{~mol}^{-1}$. Comparing the two panels, convergence between the $\mathrm{S}$ and $\mathrm{R}$ system population distributions after $t_{1}=1 \mu$ s (pink curve) is observed. 


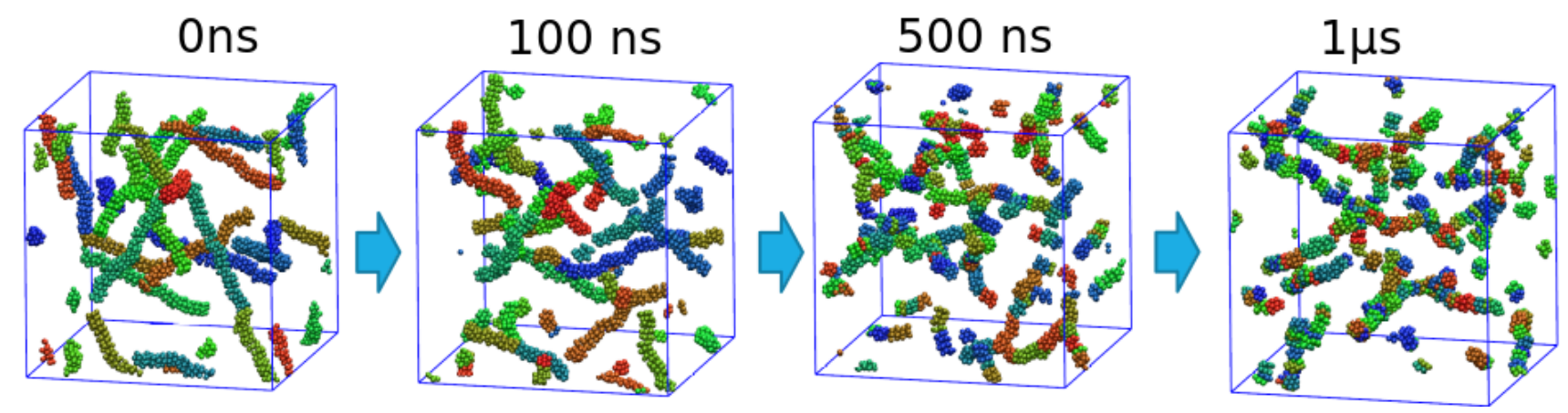

Figure S11. Snapshots of the S system $\left(\varepsilon=40 \mathrm{~kJ} \mathrm{~mol}^{-1}\right)$ at different times: at time $t_{0}$ each fiber is colored with a different color. During the MD the fibers exchange monomers and after $t_{1}=1 \mu \mathrm{s}$ the new fibers are a mixture of the initial fibers.
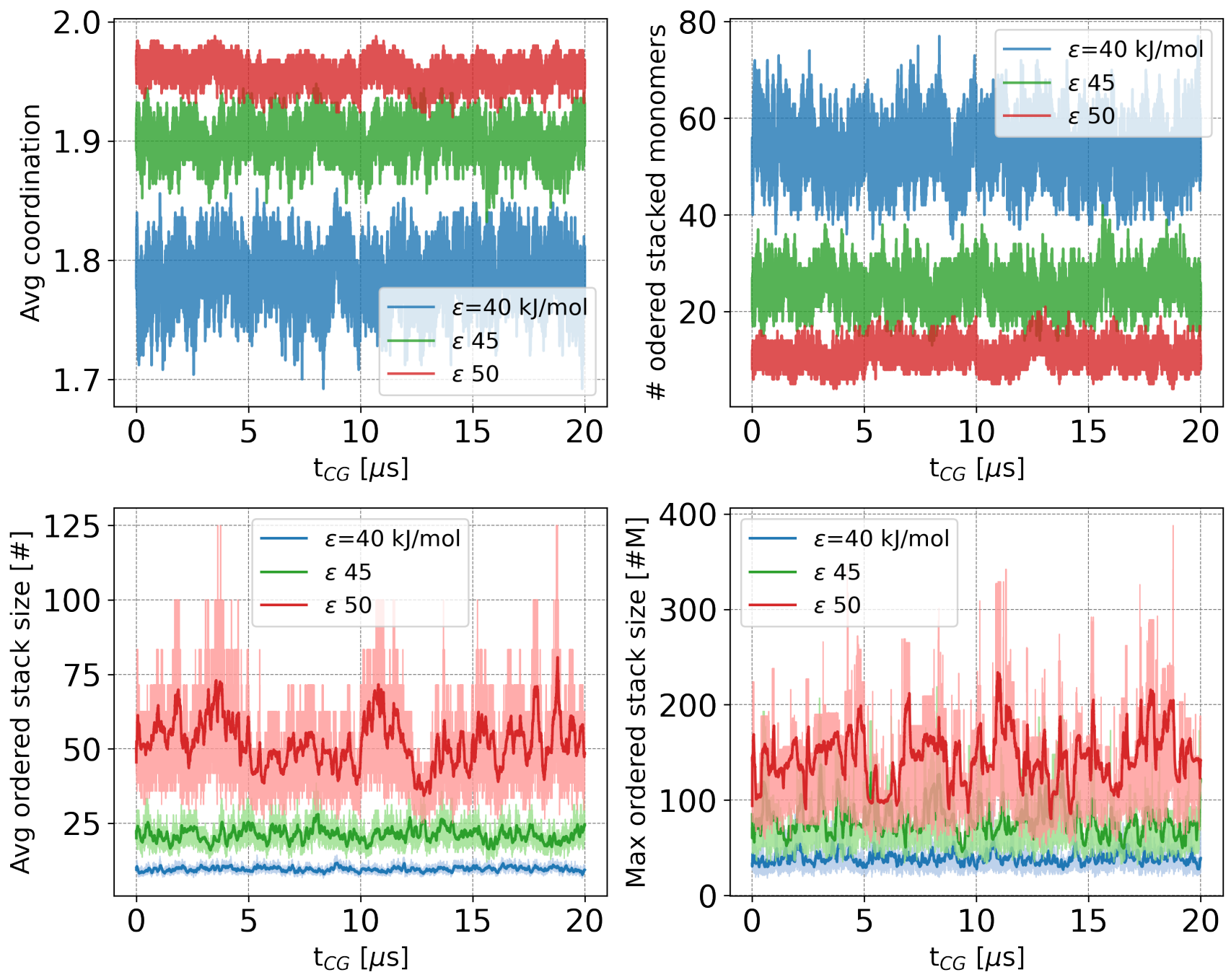

Figure S12. Self-assembly observables comparison between $\varepsilon=40 \mathrm{~kJ} \mathrm{~mol}^{-1}, 45 \mathrm{~kJ} \mathrm{~mol}^{-1}$ and $50 \mathrm{~kJ} \mathrm{~mol}^{-1} \mathbf{M}$ systems at the equilibrium: average coordination (top left), number of assemblies (top right), average size of assemblies (bottom left), size of the largest assembly (bottom right). 


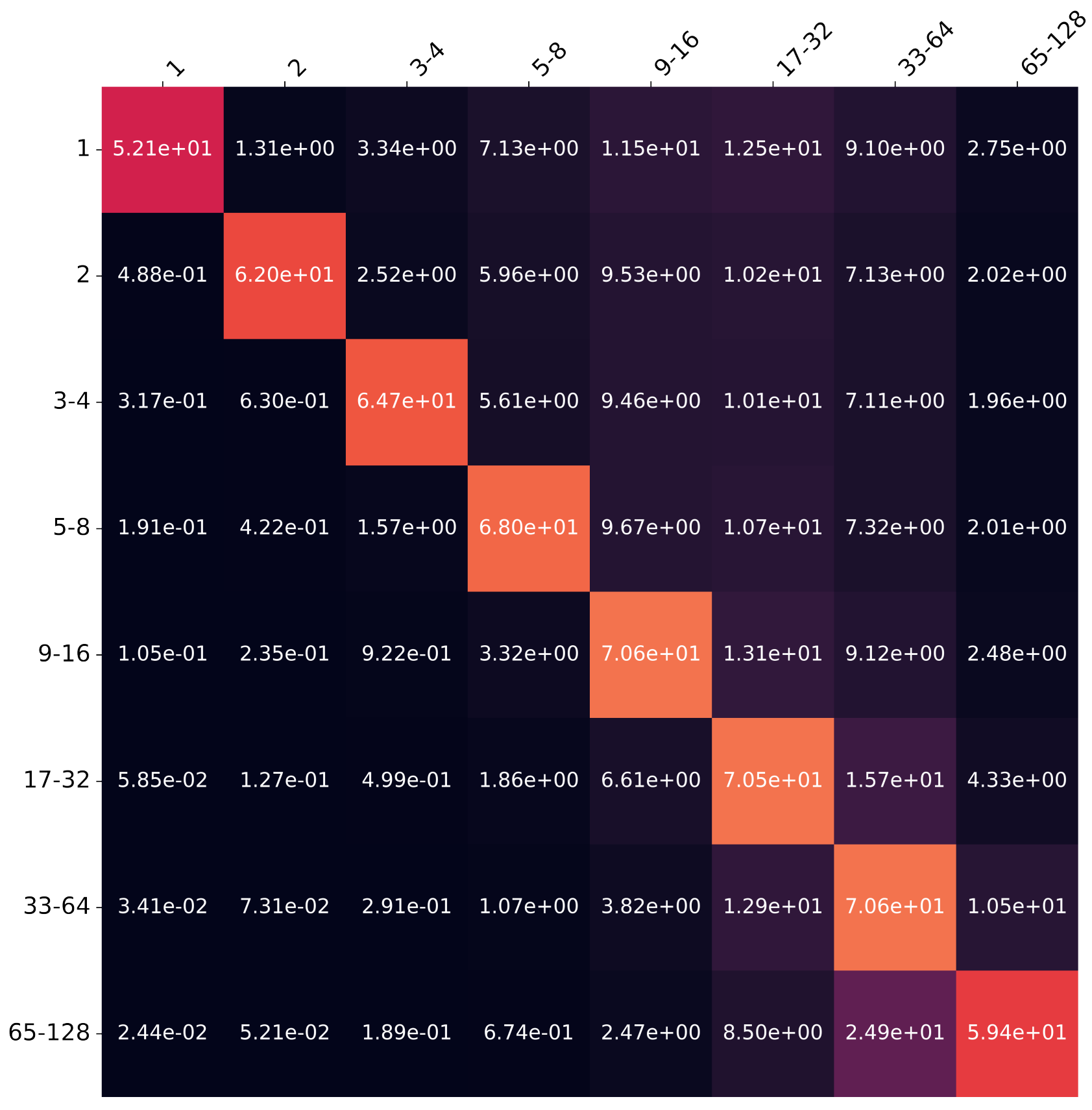

Figure S13. Sub-section of the probability transition matrix (same as reported in Figure 3a, left panel). The percentage probabilities are reported using scientific notations with three significant digits: in the main text the probabilities $<0.5$ are rounded to zero. 
a

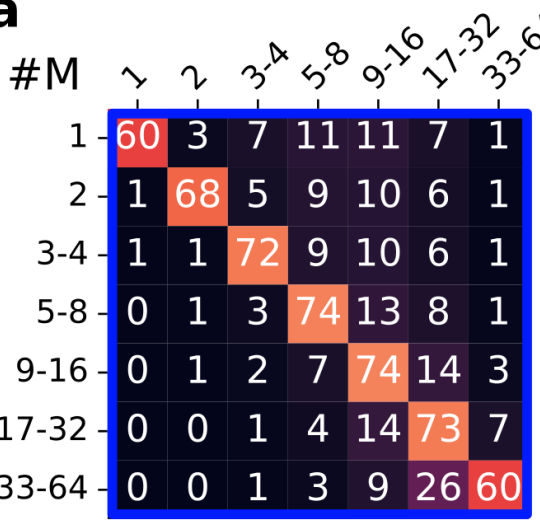

$\varepsilon=40 \mathrm{~kJ} / \mathrm{mol}$
RAW TRANSITION DATA

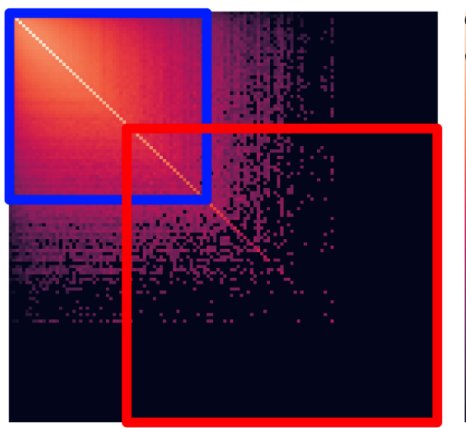

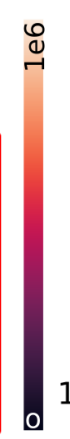

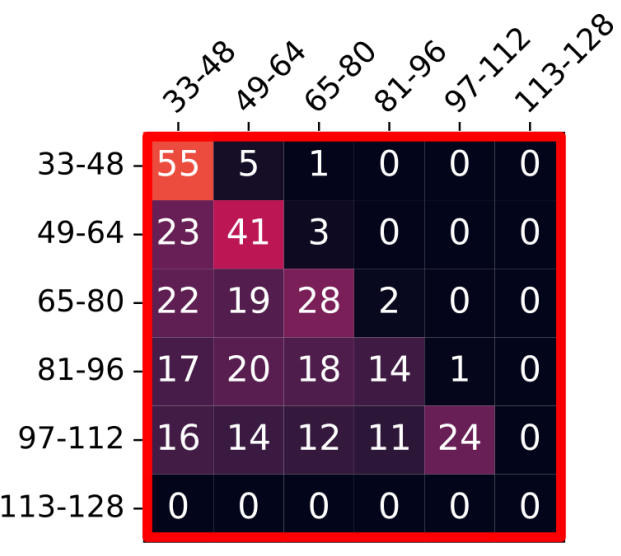

b

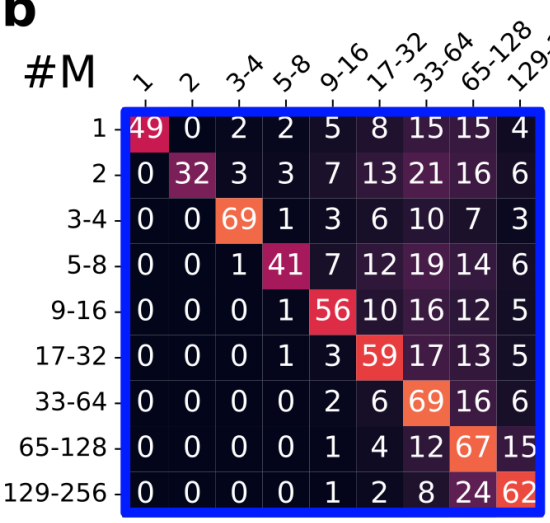

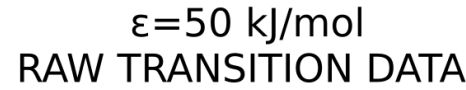

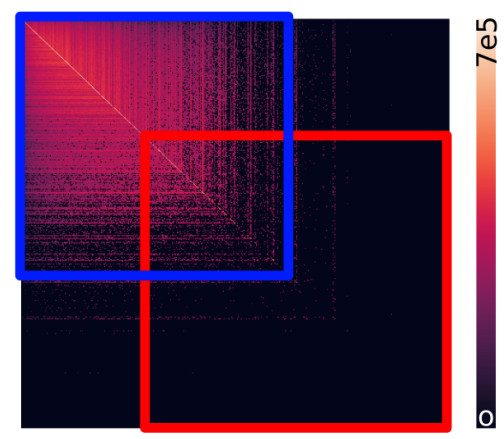

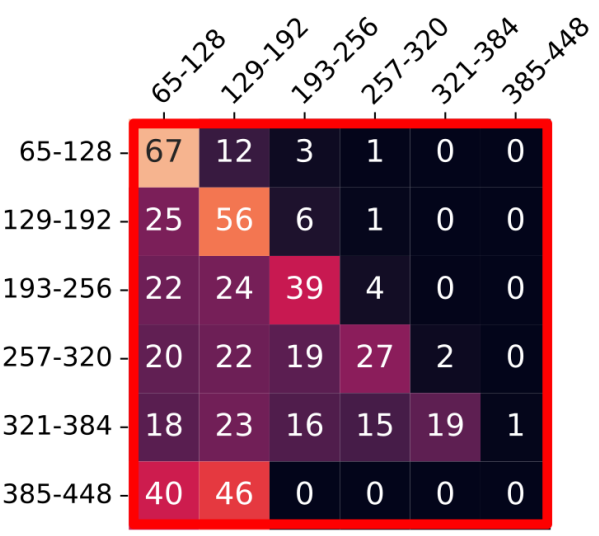

Figure S14. Transition matrices for the M-model at $\varepsilon=40 \mathrm{~kJ} \mathrm{~mol}^{-1}$ (top) and at $\varepsilon=50 \mathrm{~kJ} \mathrm{~mol}^{-1}$ (bottom). Each entry $(i, j)$ of the raw transition matrices (central column) shows how many monomers transit from an assembly of size $i$ to an assembly of size $j$ every $\Delta \tau=300 \mathrm{ps}$ of CG-MD time; The left and right panels report two sub-regions of the transition probability matrix (red and blue rectangles). Here, the size of the aggregates are grouped for clarity. The numbers in the cells indicate the percentage probability (the 0 s identify transitions with probability $<0.5$ ). The raw transition matrices are colored in $\log$ scale. 
HEX \& 40

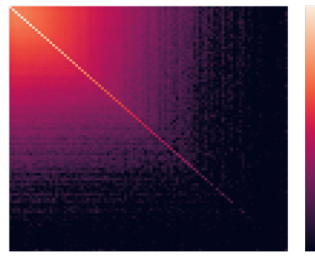

P:

\section{HEX \& 45}

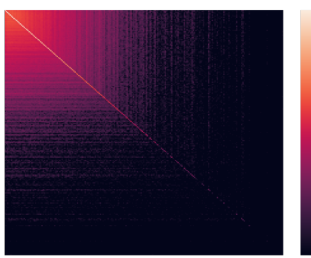

P:

HEX \& 50

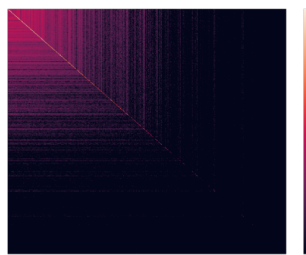

P:

$3 \%$

\section{$A=21 E=4$}

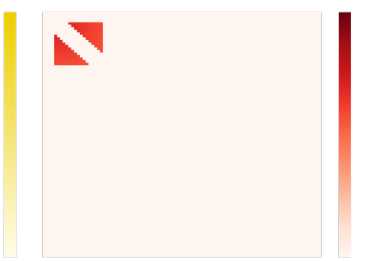

$18 \%$

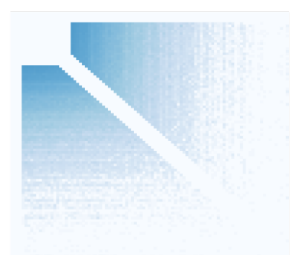

$22 \%$
$8 \%$

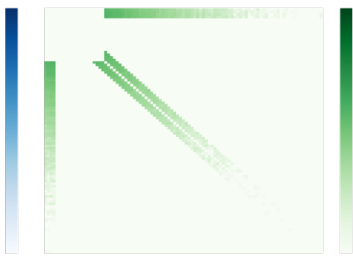

Figure S15. Assembly transition matrices for the $\mathbf{M}$ systems (left), decomposed into areas identifying different classes of polymerisation/depolymerisation mechanisms (see Methods for details). The areas are defined by the parameters $A=21$ and $E=\langle A\rangle / 5 \approx 4$, as explained in the main text. The percentage is computed as the sum of each entry of the matrix in the considered area divided by the sum of all the entries of the matrix, without considering the diagonal: this gives an estimate of the predominant mechanism by which the system communicates. The obtained percentage are reported under each areas. 

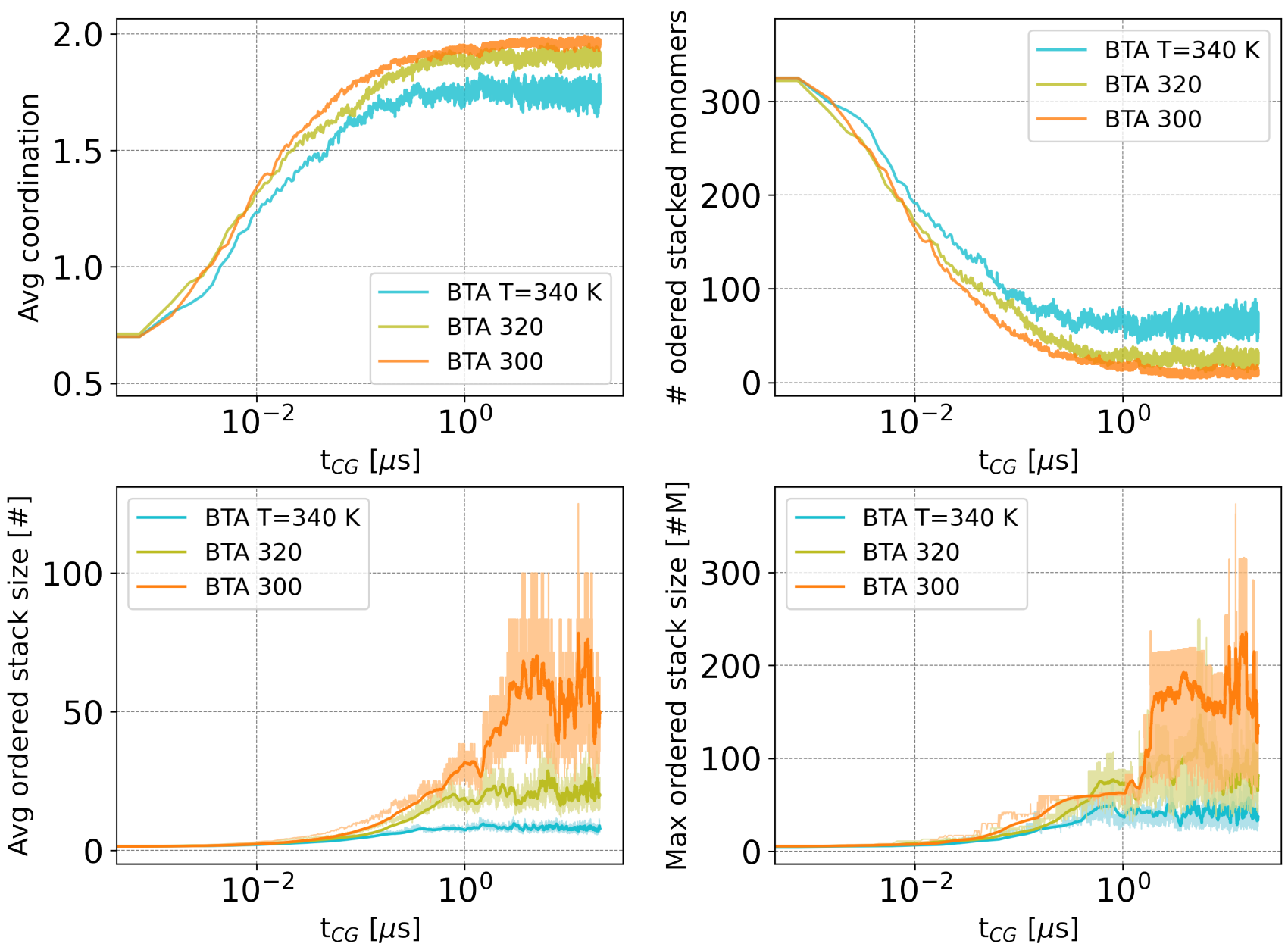

Figure S16. Self-assembly observables comparison between $T=340 \mathrm{~K}, T=320 \mathrm{~K}$ and $T=300 \mathrm{~K}$ BTA system starting from random conformations in log scale: average coordination (top left), number of assemblies (top right), average size of assemblies (bottom left), size of the largest assembly (bottom right). 

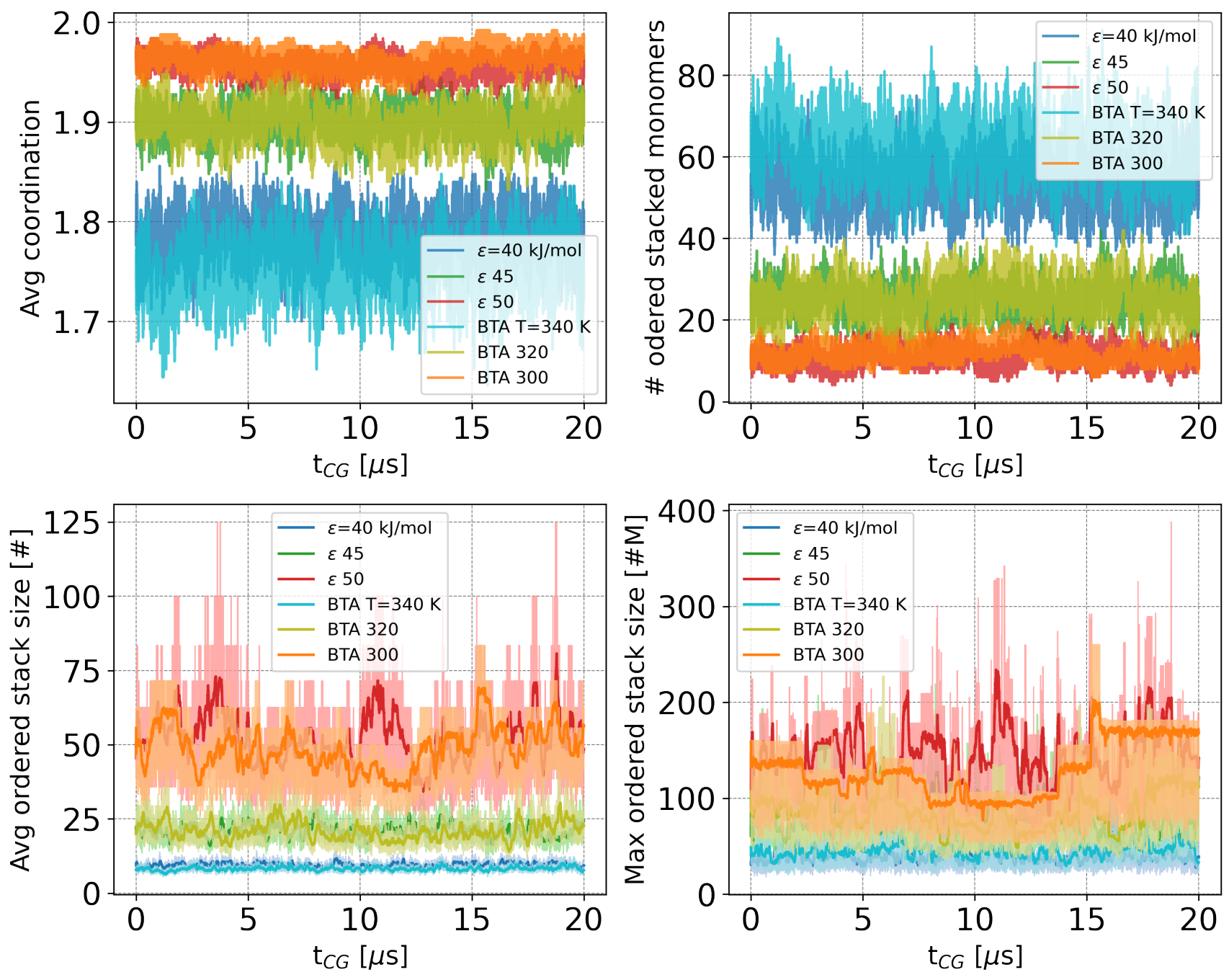

Figure S17. Self-assembly observables comparison between $T=340 \mathrm{~K}, T=320 \mathrm{~K}$ and $T=300 \mathrm{~K}$ BTA system and $\varepsilon=40 \mathrm{~kJ} \mathrm{~mol}^{-1}, 45 \mathrm{~kJ} \mathrm{~mol}^{-1}$ and $50 \mathrm{~kJ} \mathrm{~mol}^{-1} \mathrm{M}$ systems: average coordination (top left), number of assemblies (top right), average size of assemblies (bottom left), size of the largest assembly (bottom right). 


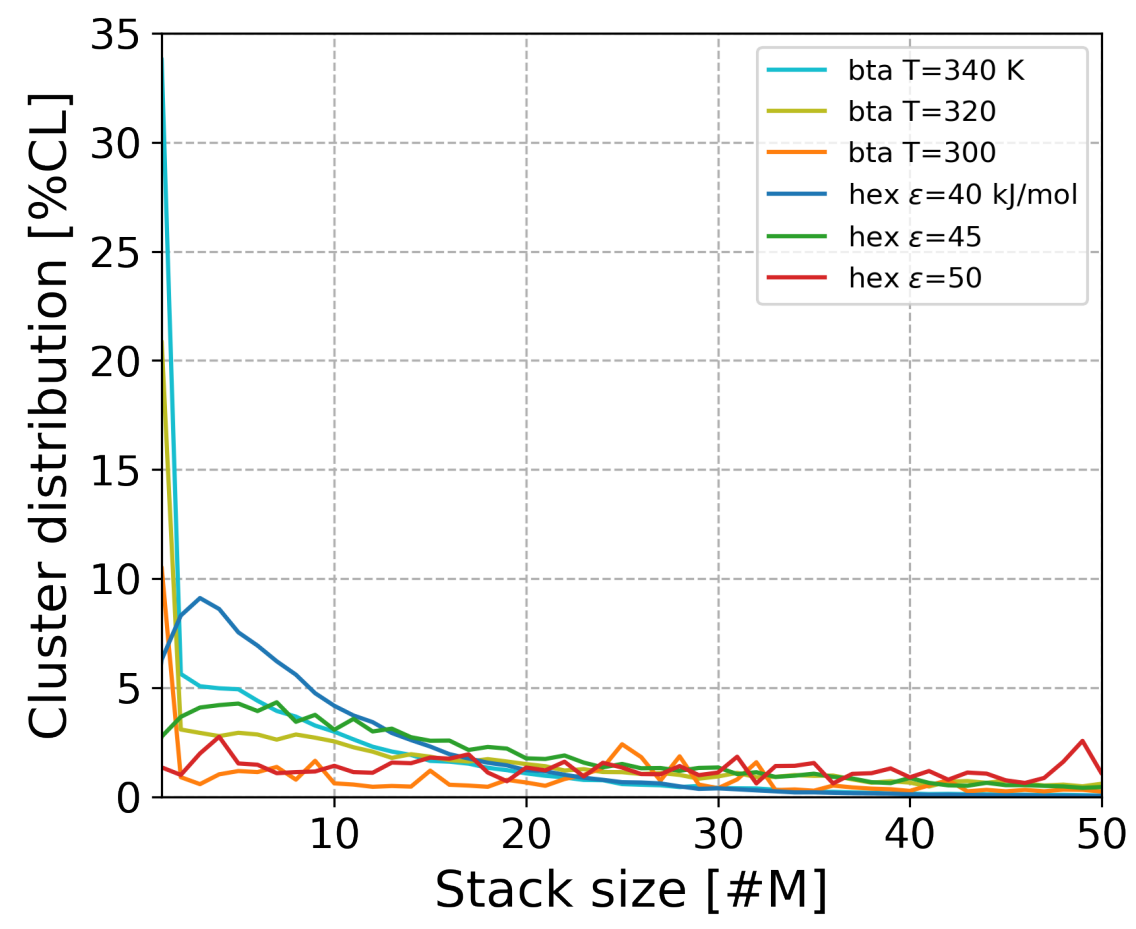

Figure S18. Assembly distributions comparison between $\varepsilon=40 \mathrm{~kJ} \mathrm{~mol}^{-1}, 45 \mathrm{~kJ} \mathrm{~mol}^{-1}$ and $50 \mathrm{~kJ} \mathrm{~mol}^{-1} \mathbf{M}$ systems and $T=340 \mathrm{~K}, T=320 \mathrm{~K}$ and $T=300 \mathrm{~K}$ BTA system.
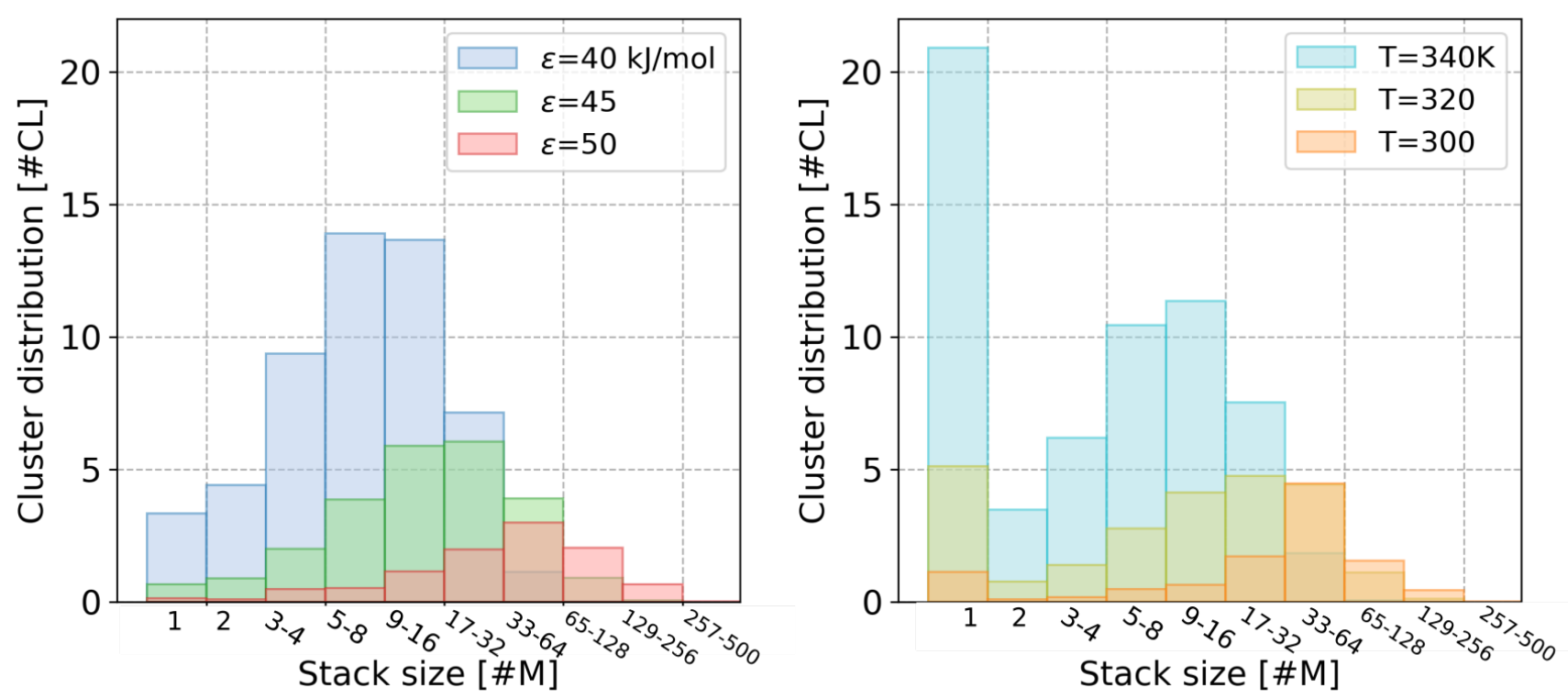

Figure S19. Distribution of assemblies of different sizes over the total number of assemblies. Comparison between $\varepsilon=40 \mathrm{~kJ} \mathrm{~mol}^{-1}, 45 \mathrm{~kJ} \mathrm{~mol}^{-1}$ and $50 \mathrm{~kJ} \mathrm{~mol}^{-1} \mathbf{M}$ systems (left) and $T=340 \mathrm{~K}, T=320 \mathrm{~K}$ and $T=300 \mathrm{~K} \mathrm{BTA}$ system (right): the sizes are grouped in binary size-ranges and reported in log scale along the $\mathrm{x}$ axis. 

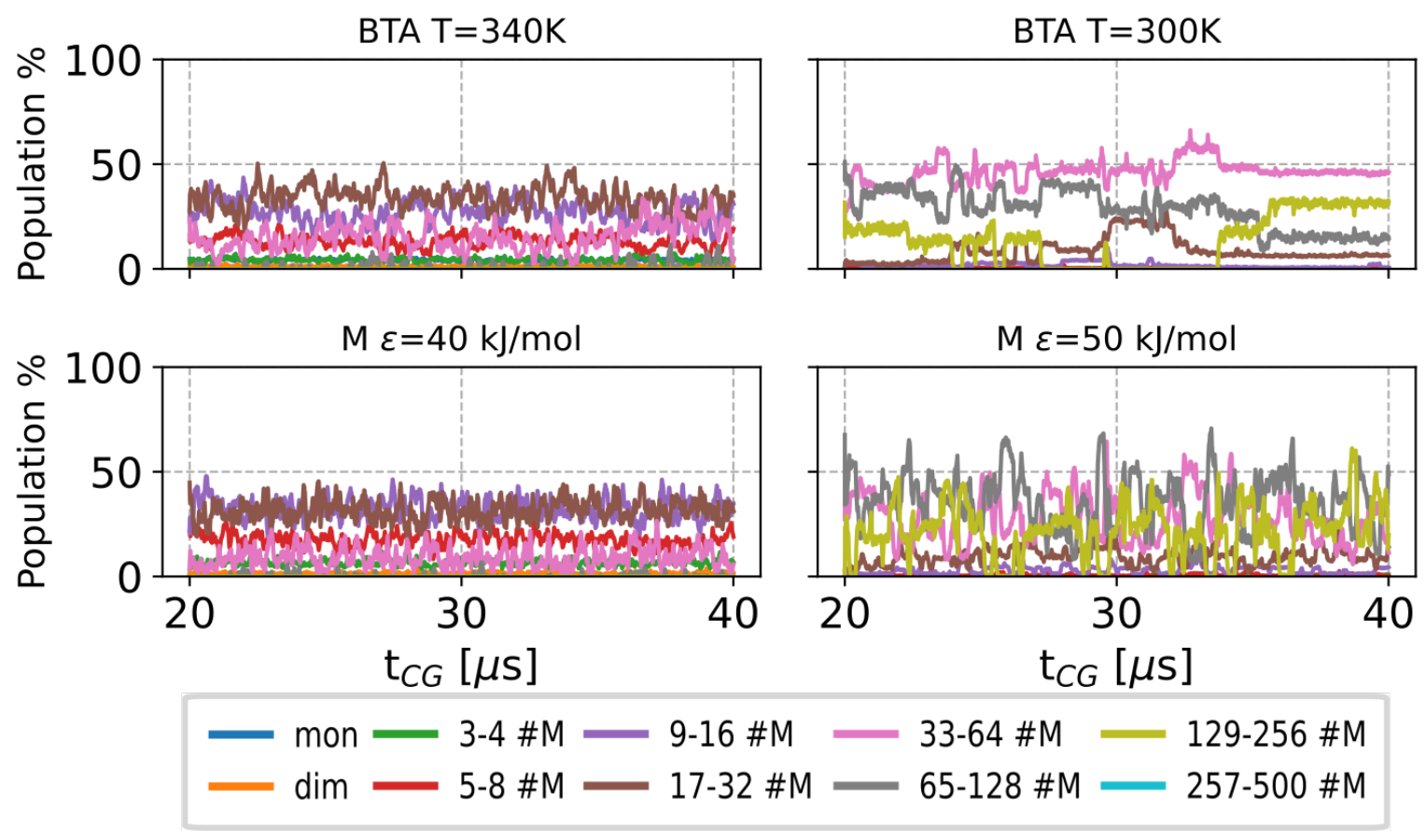

Figure S20. Percentage of monomers belonging to different size aggregates for the BTA systems at $T=340 \mathrm{~K}$ and $T=300 \mathrm{~K}$ and $\mathbf{M}$ system $\varepsilon=40 \mathrm{~kJ} \mathrm{~mol}^{-1}$ and $50 \mathrm{~kJ} \mathrm{~mol}^{-1}$ 

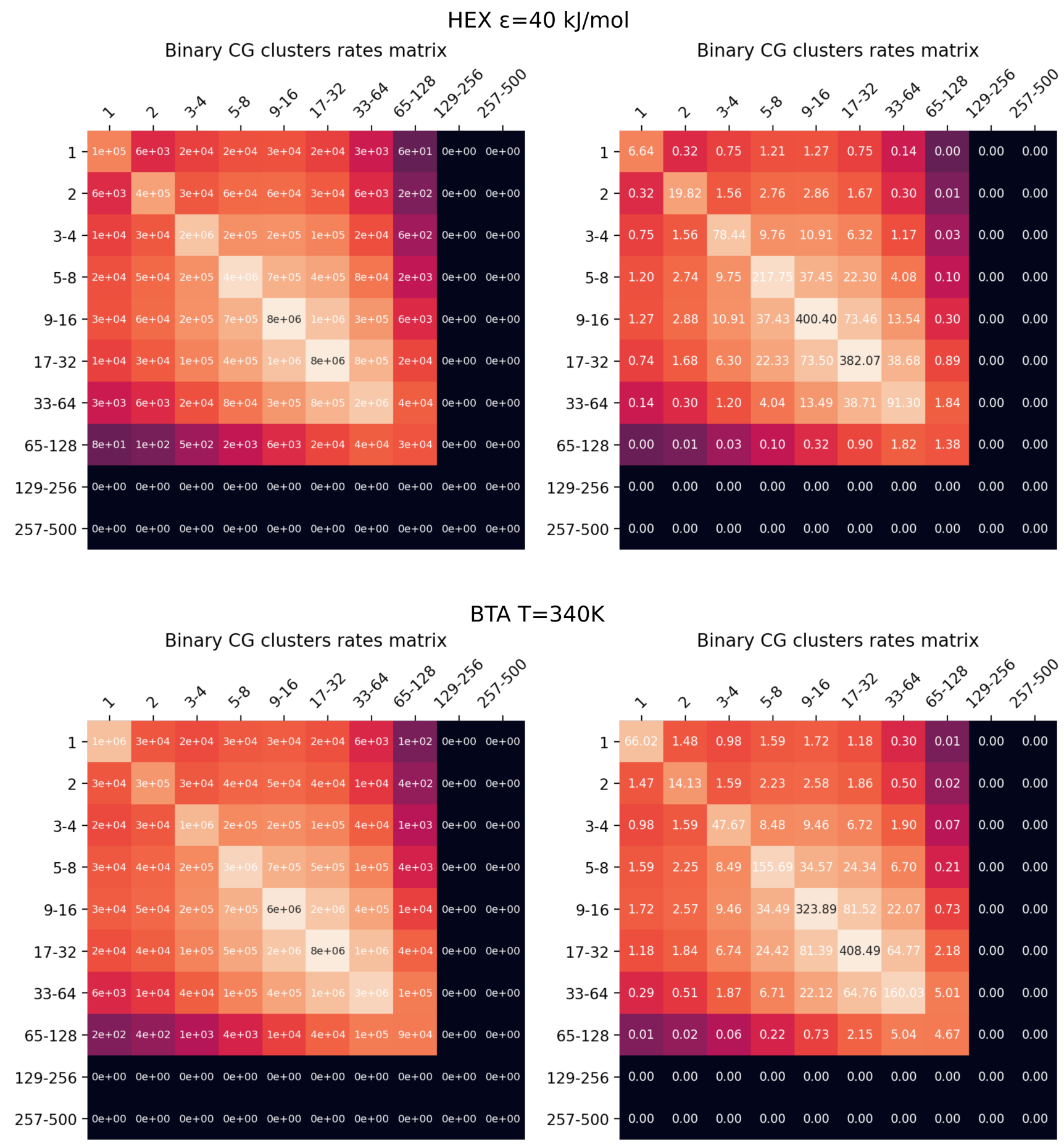

Figure S21. M with $\varepsilon=40 \mathrm{~kJ} \mathrm{~mol}^{-1}$ (top), and $T=340 \mathrm{~K}$ (bottom): raw transition matrices grouped in binary size-ranges (left) and transition rate matrices in $\mathrm{ns}^{-1}$, obtained from raw data matrices divided by the trajectory time-length (right), both colored in log scale. 


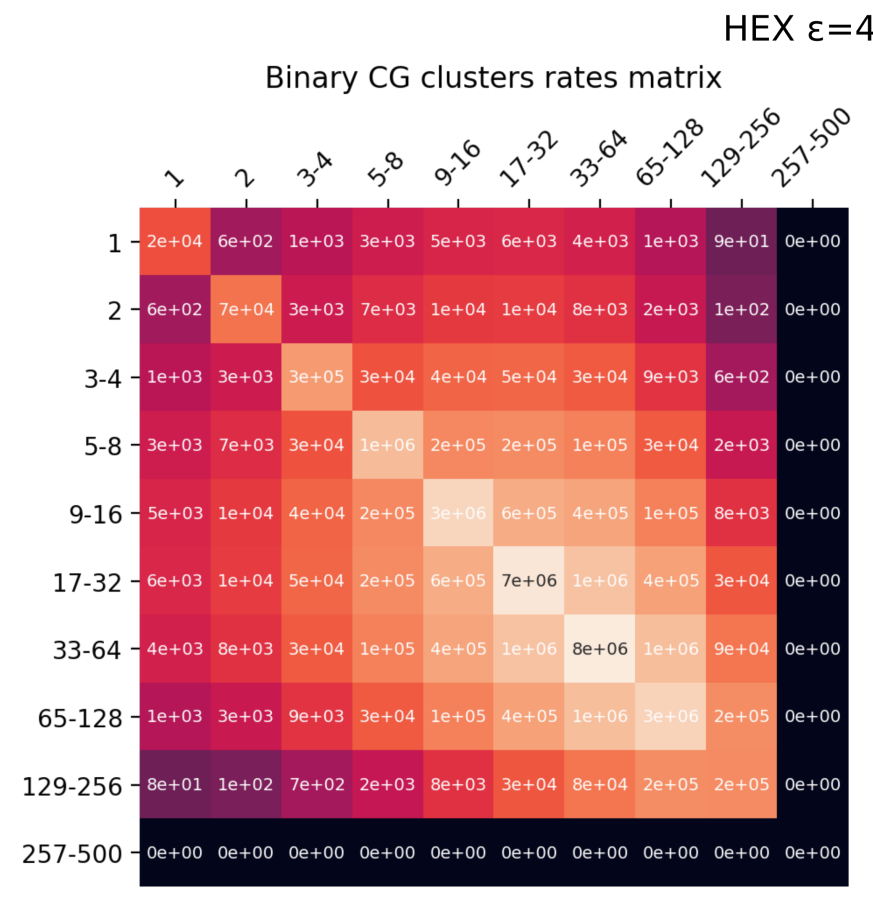

$\mathrm{kJ} / \mathrm{mol}$
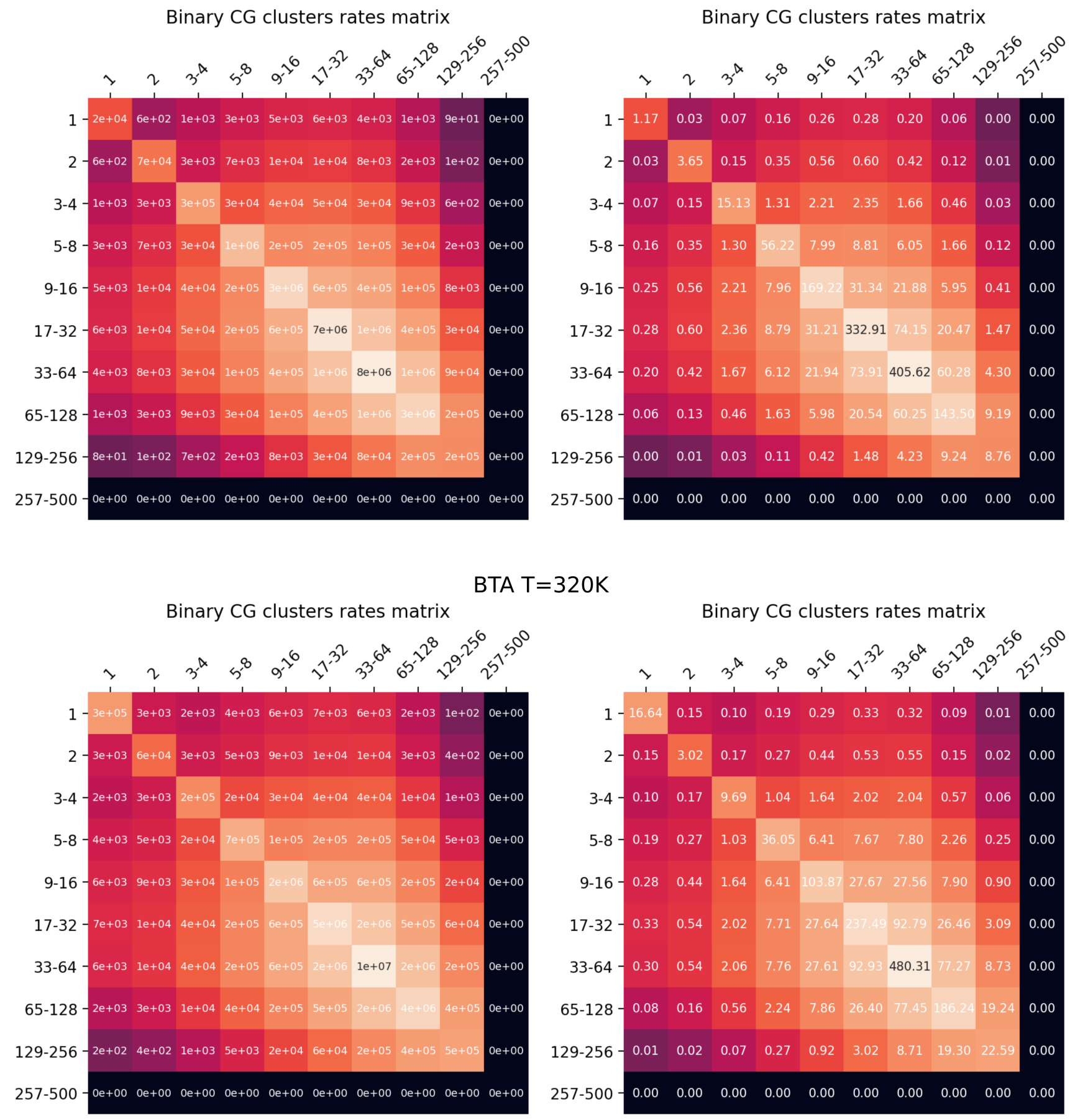

Figure S22. M with $\varepsilon=45 \mathrm{~kJ} \mathrm{~mol}^{-1}$ (top), and $T=320 \mathrm{~K}$ (bottom): raw transition matrices grouped in binary size-ranges (left) and transition rate matrices in $\mathrm{ns}^{-1}$, obtained from raw data matrices divided by the trajectory time-length (right), both colored in log scale. 


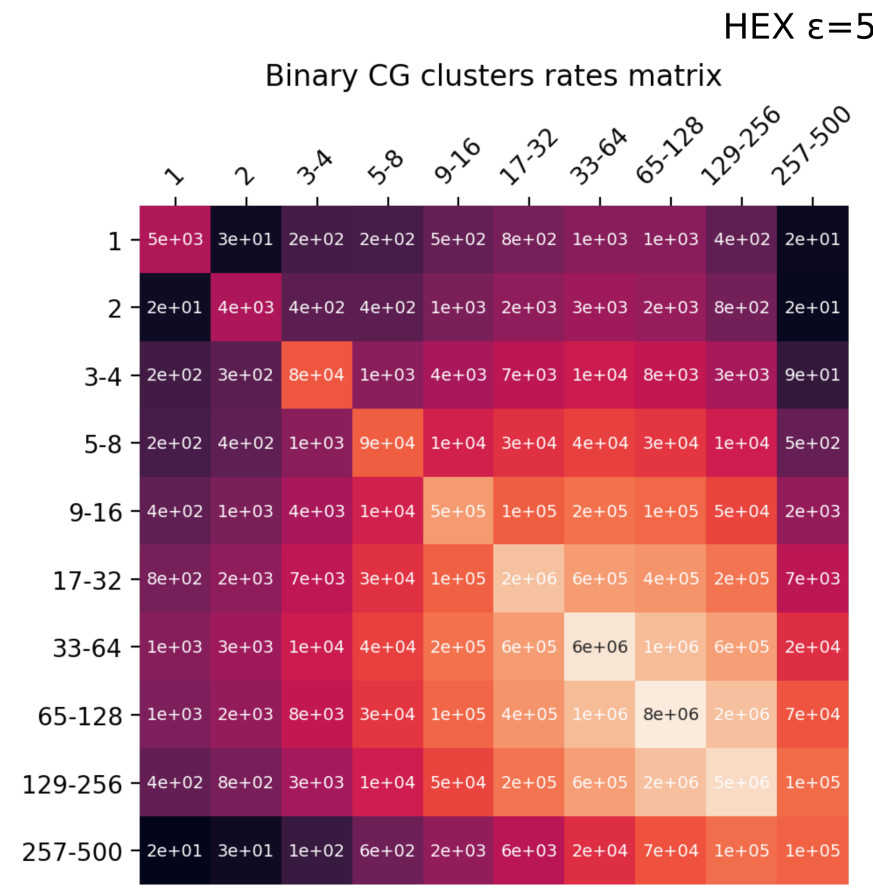

$\mathrm{kJ} / \mathrm{mol}$
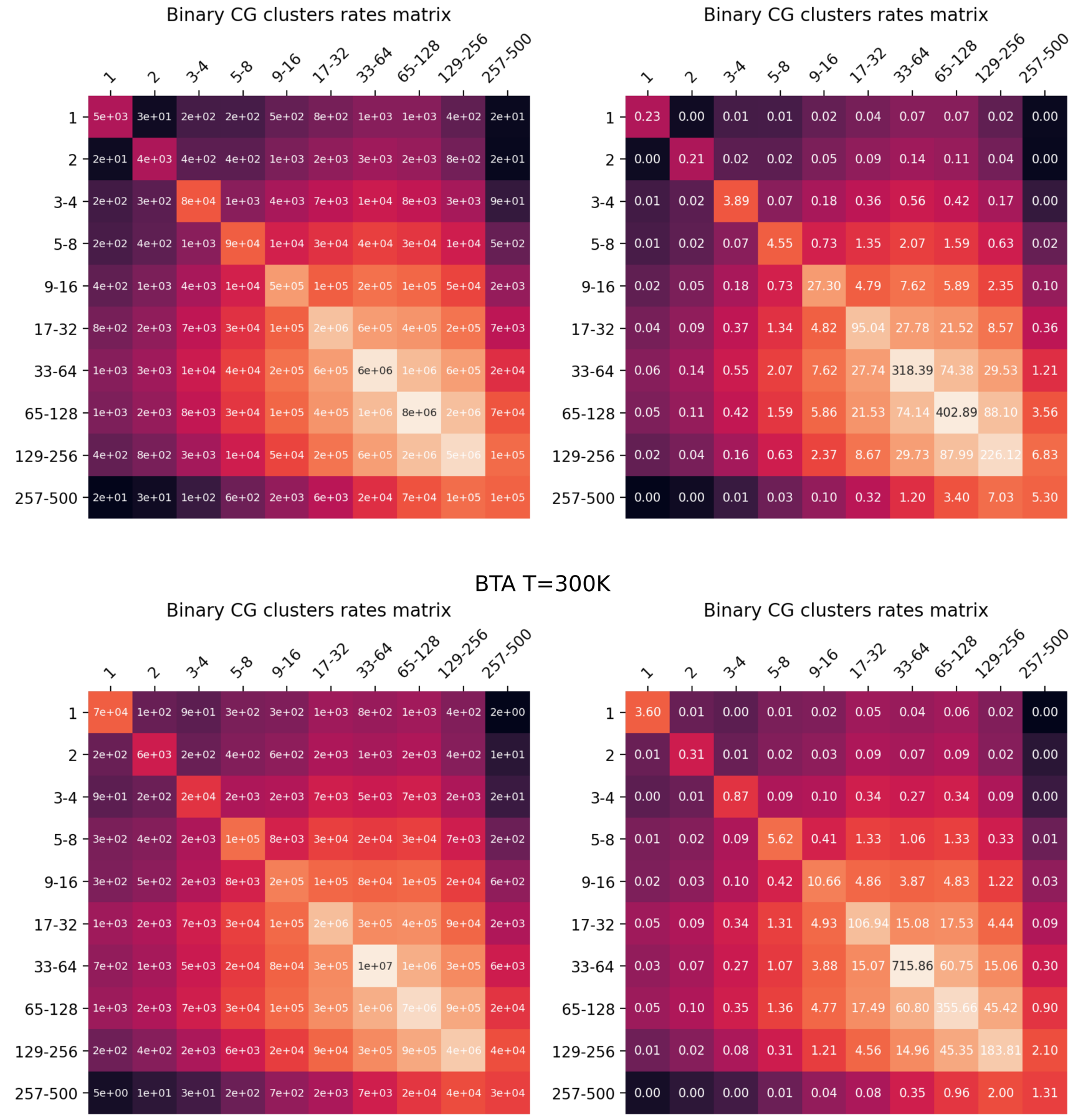

Figure S23. M with $\varepsilon=50 \mathrm{~kJ} \mathrm{~mol}^{-1}$ (top), and $T=300 \mathrm{~K}$ (bottom): raw transition matrices grouped in binary size-ranges (left) and transition rate matrices in $\mathrm{ns}^{-1}$, obtained from raw data matrices divided by the trajectory time-length (right), both colored in log scale. 
a

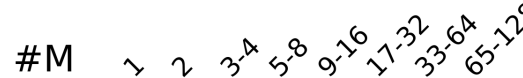

$$
\begin{aligned}
& \begin{array}{lllllllll}
1 & 52 & 1 & 3 & 7 & 12 & 13 & 9 & 3
\end{array} \\
& \begin{array}{lllllllll}
2 & 0 & 62 & 3 & 6 & 10 & 10 & 7 & 2
\end{array} \\
& \begin{array}{lllllllll}
3-4 & 0 & 1 & 65 & 6 & 9 & 10 & 7 & 2
\end{array} \\
& \text { 5-8 } 00 \begin{array}{lllllllll} 
& 0 & 2 & 68 & 10 & 11 & 7 & 2
\end{array} \\
& \begin{array}{ccccccccc}
9-16 & 0 & 0 & 1 & 3 & 71 & 13 & 9 & 2
\end{array} \\
& \text { 17-32 } 00 \begin{array}{llllllll} 
& 0 & 0 & 2 & 7 & 70 & 16 & 4
\end{array} \\
& \text { 33-64 } 00 \begin{array}{llllllll} 
& 0 & 0 & 1 & 4 & 13 & 71 & 10
\end{array} \\
& \begin{array}{lllllllll}
65-128 & 0 & 0 & 0 & 1 & 2 & 8 & 25 & 59
\end{array}
\end{aligned}
$$

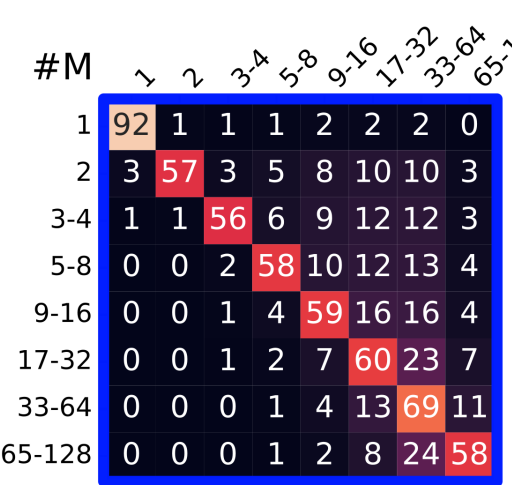
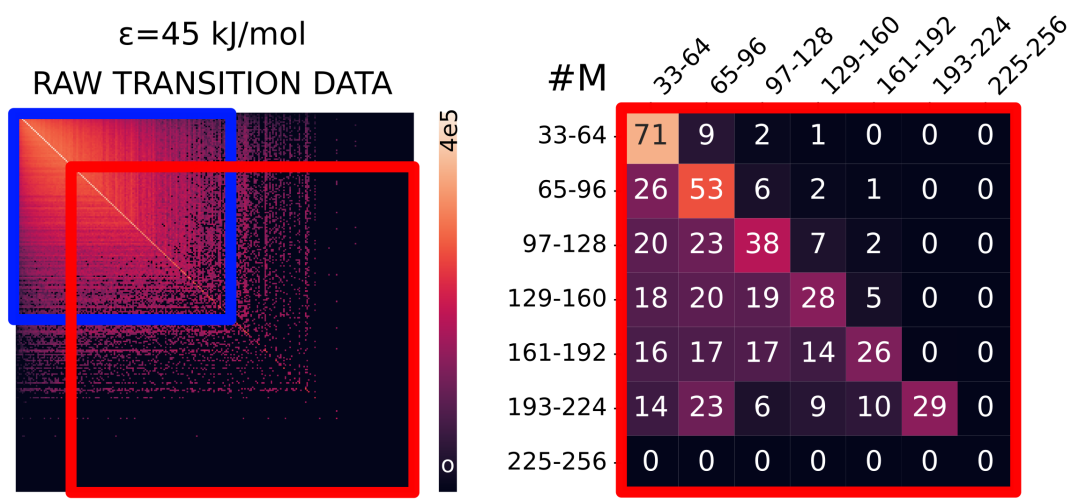

Figure S24. Comparison between BTA $T=320 \mathrm{~K}$ (bottom) and $\mathbf{M}$ with $\varepsilon=45 \mathrm{~kJ} \mathrm{~mol}^{-1}$ (top). The raw transition matrix (center) and transition probability sub-matrices (left and right panels, the blue and red rectangles indicate the region highlighted) are reported. Here, the size of the aggregates are grouped for clarity. The numbers in the cells indicate the percentage probability (the 0 s identify transitions with probability $<0.5$ ). The transition matrices are colored in log scale. 
HEX \& 40

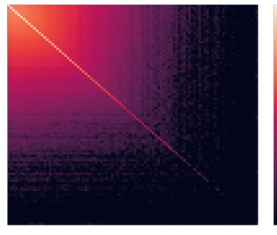

P:

BTA T 340

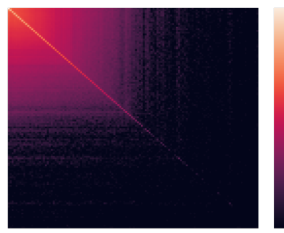

P:

HEX \& 45

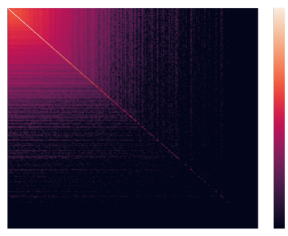

P:

BTA T 320

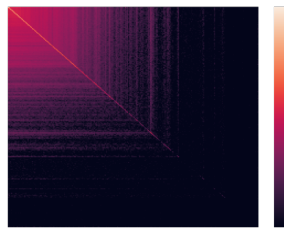

P:

HEX \& 50

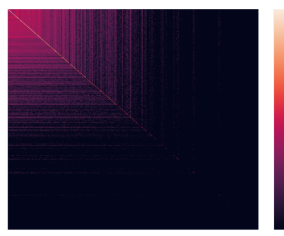

P:

BTA T 300

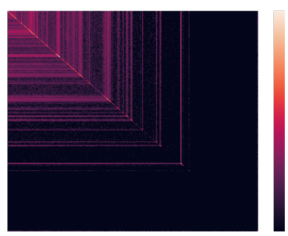

P:

\section{$A=21 E=4$}

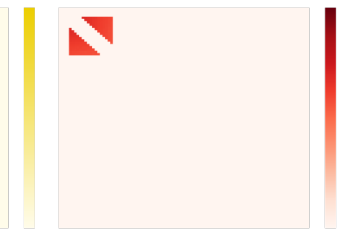

$18 \%$

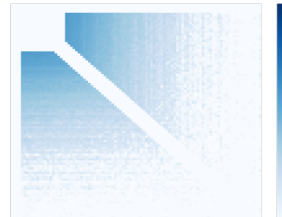

$22 \%$

$8 \%$
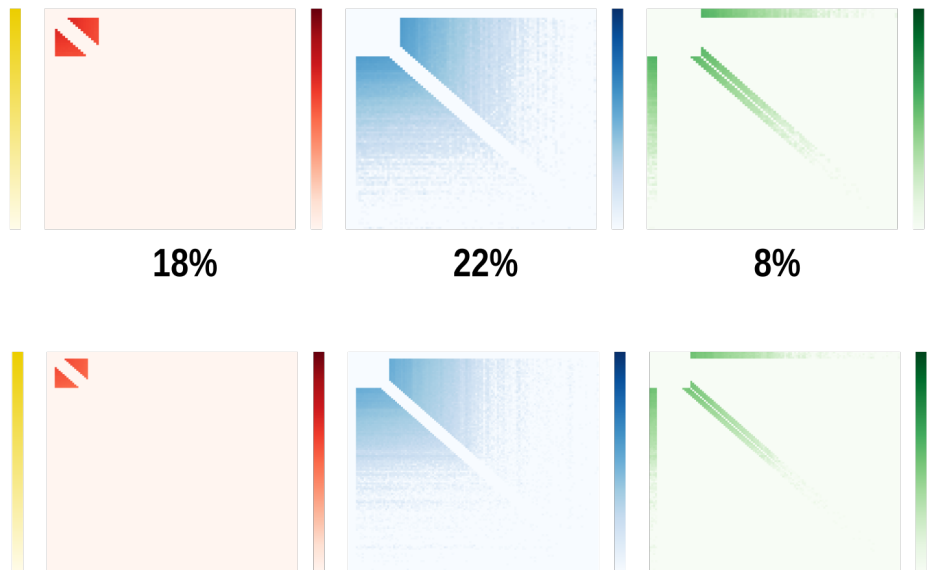

$16 \%$

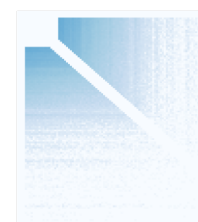

$26 \%$

$10 \%$

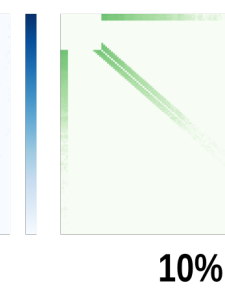

\section{$A=21 E=4$}

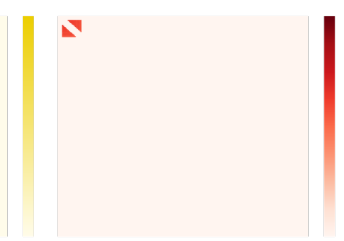

$10 \%$

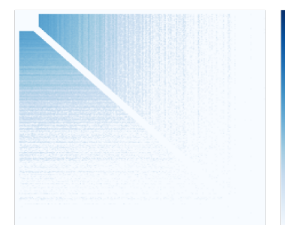

$58 \%$

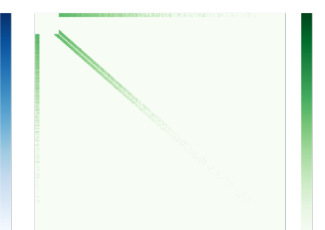

$14 \%$

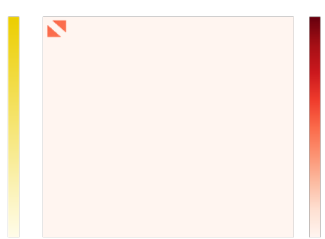

$7 \%$

$63 \%$
$14 \%$

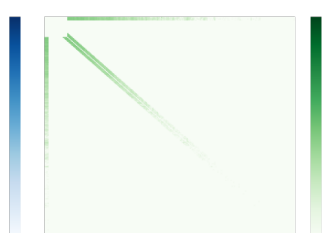

\section{$A=21 E=4$}

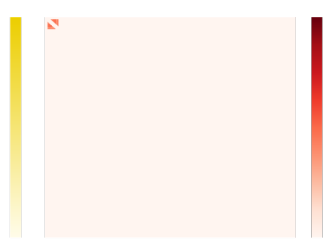

$2 \%$

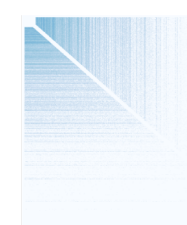

$84 \%$

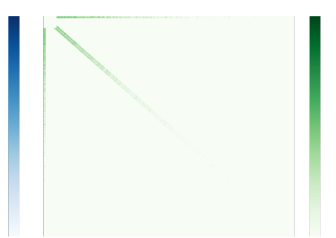

$11 \%$

$3 \%$

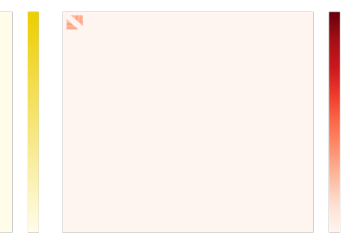

$1 \%$

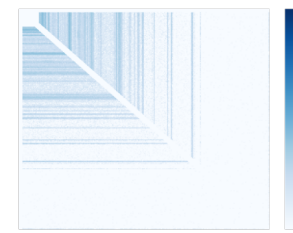

$83 \%$

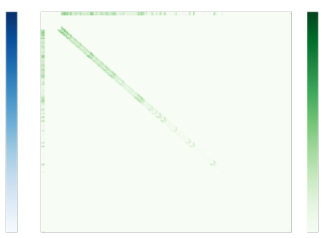

$13 \%$

Figure S25. Assembly transition matrices (see Methods for details): comparison between $\mathbf{M}$ system and BTA system for $A=21$ and $E=\langle A\rangle / 5 \approx 4$. 
HEX \& 40

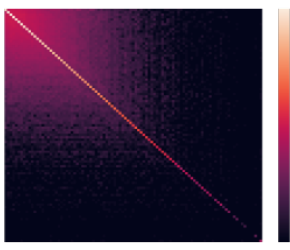

P:

BTA T 340

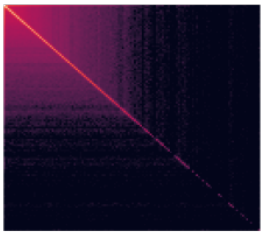

P:

HEX \& 45

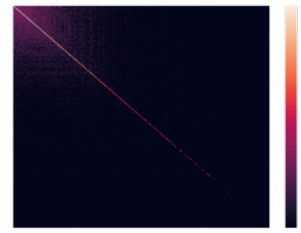

P:

BTA T 320

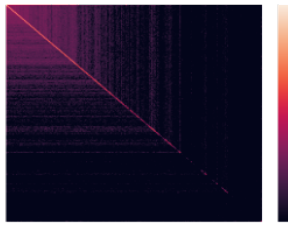

P:

HEX \& 50

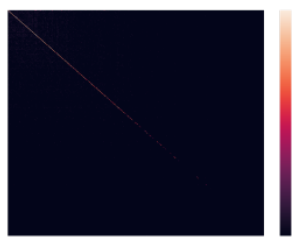

P:

BTA T 300

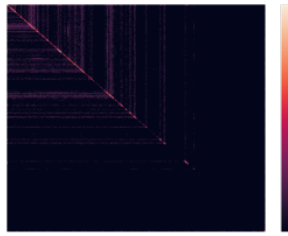

P:

$3 \%$

$43 \%$

$13 \%$

$2 \%$

$A=21 E=4$

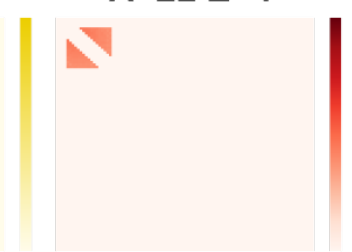

$17 \%$

$30 \%$

$10 \%$
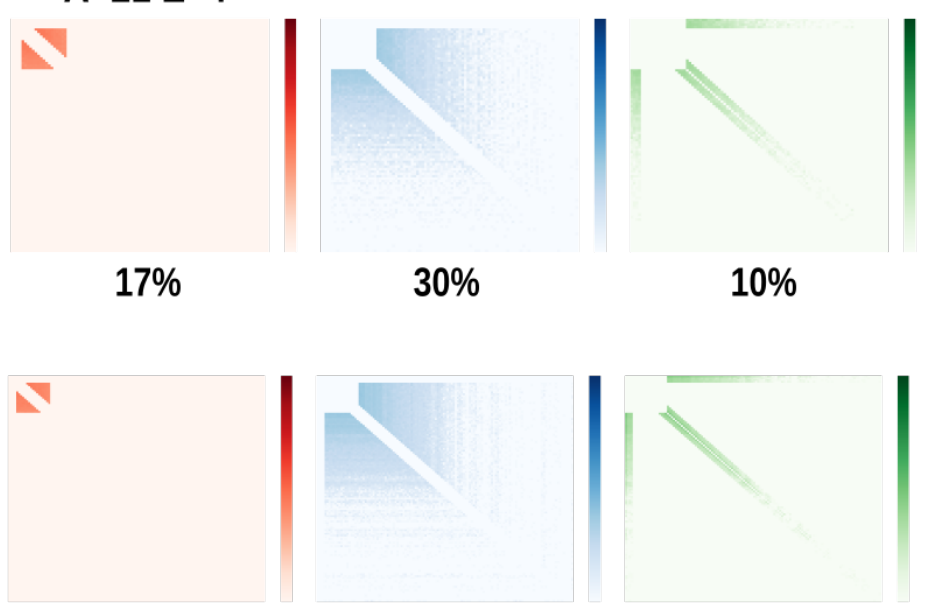

$9 \%$

$25 \%$

$14 \%$
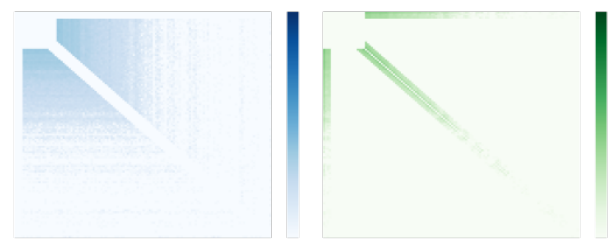

$A=21 E=4$

$7 \%$

$65 \%$

$15 \%$
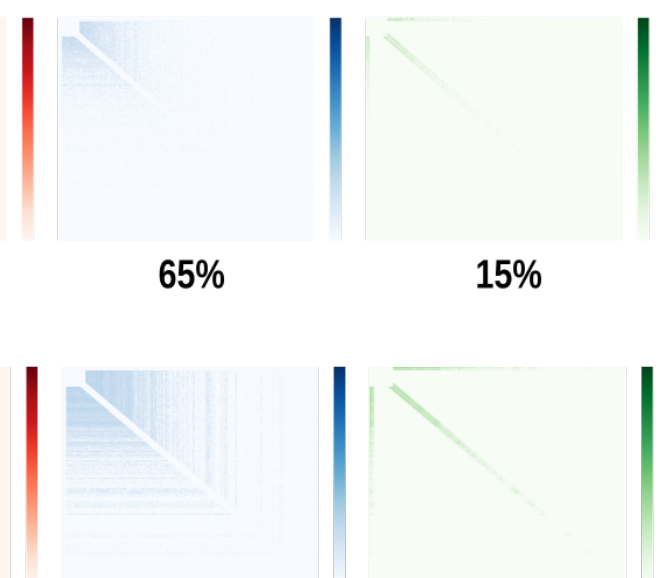

$4 \%$

$60 \%$

$19 \%$

\section{$A=21 E=4$}

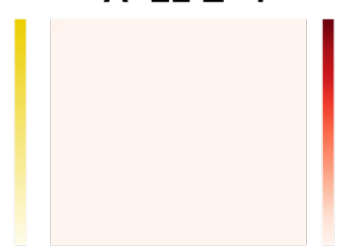

$1 \%$

$82 \%$

$14 \%$
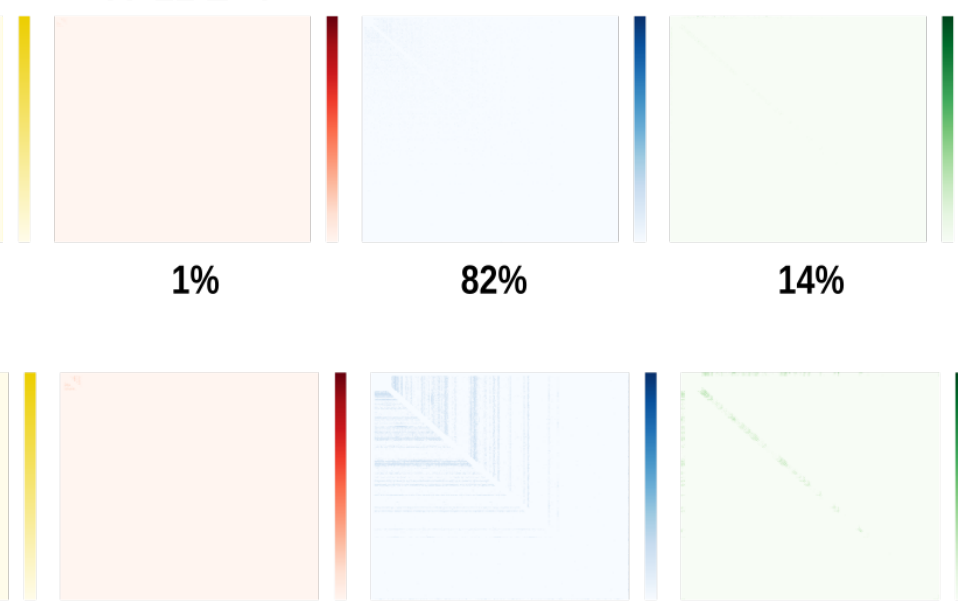

$1 \%$

$76 \%$

$20 \%$

Figure S26. Assembly transition matrices (see Methods for details): comparison between $\mathbf{M}$ system and BTA system for $A=21$ and $E=\langle A\rangle / 5 \approx 4$ with cut-off radius $r_{\text {rcut }}=0.7 \mathrm{~nm}$. 
HEX \& 40

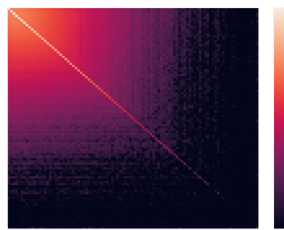

P:

BTA T 340

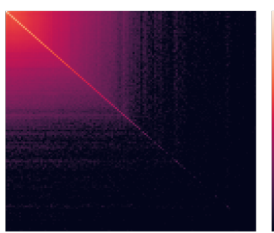

P:

$19 \%$

HEX \& 45

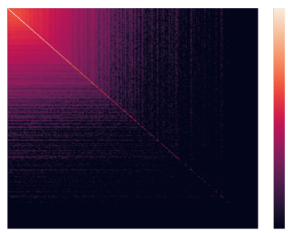

P:

BTA T 320

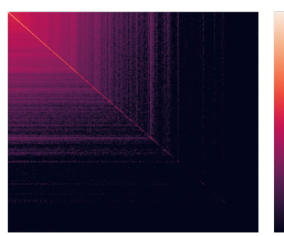

P:

HEX \& 50

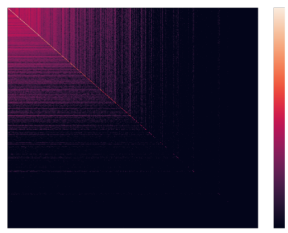

P:

\section{BTA T 300}

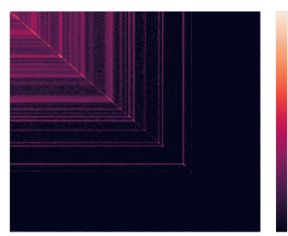

P:

$18 \%$

$18 \%$

$18 \%$

$19 \%$
$A=9 E=2$

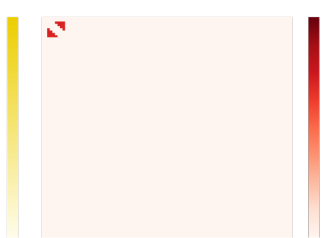

$7 \%$

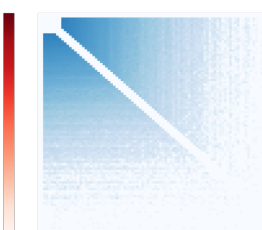

$59 \%$

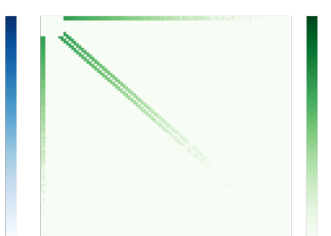

$16 \%$

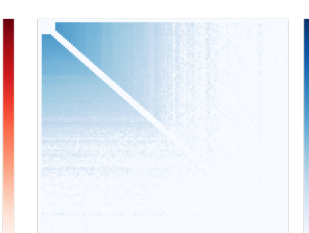

$58 \%$

$18 \%$

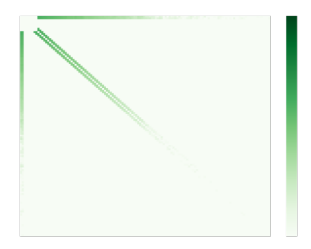

$5 \%$

\section{$A=21 E=4$}

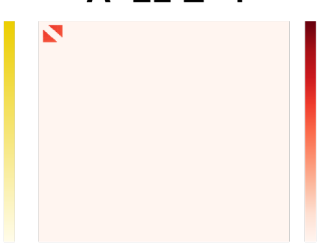

$10 \%$

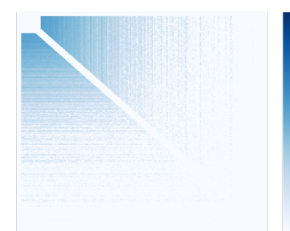

$58 \%$

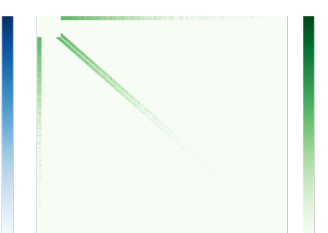

$14 \%$

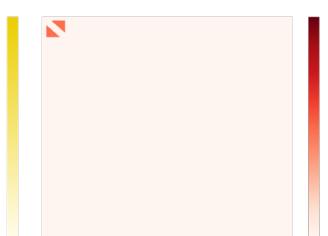

$7 \%$

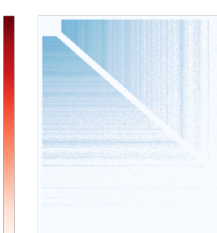

$63 \%$

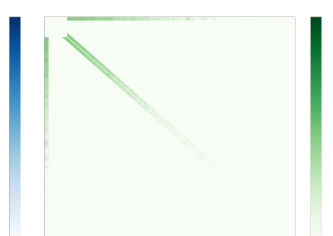

$14 \%$

\section{$A=50 E=10$}

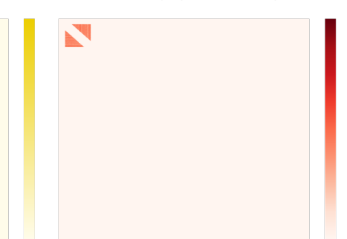

$10 \%$

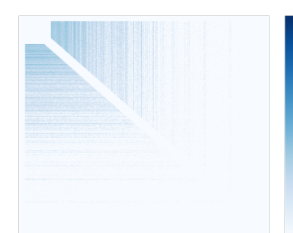

$56 \%$

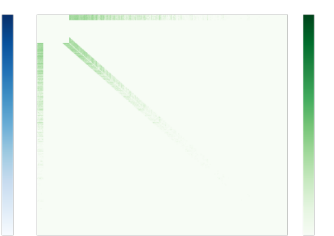

$15 \%$

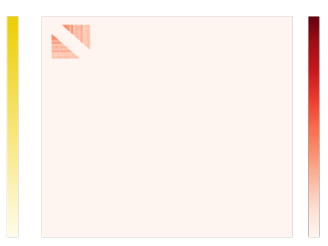

$6 \%$

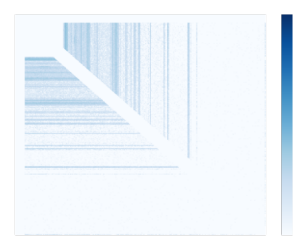

$57 \%$

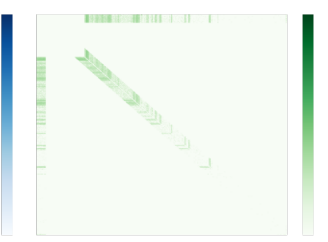

$18 \%$

Figure S27. Assembly transition matrices (see Methods for details): comparison between $\mathbf{M}$ system and BTA system for $A=\langle$ size $\rangle$, namely the average size relative to the system under study and $E=\langle A\rangle / 5$. 


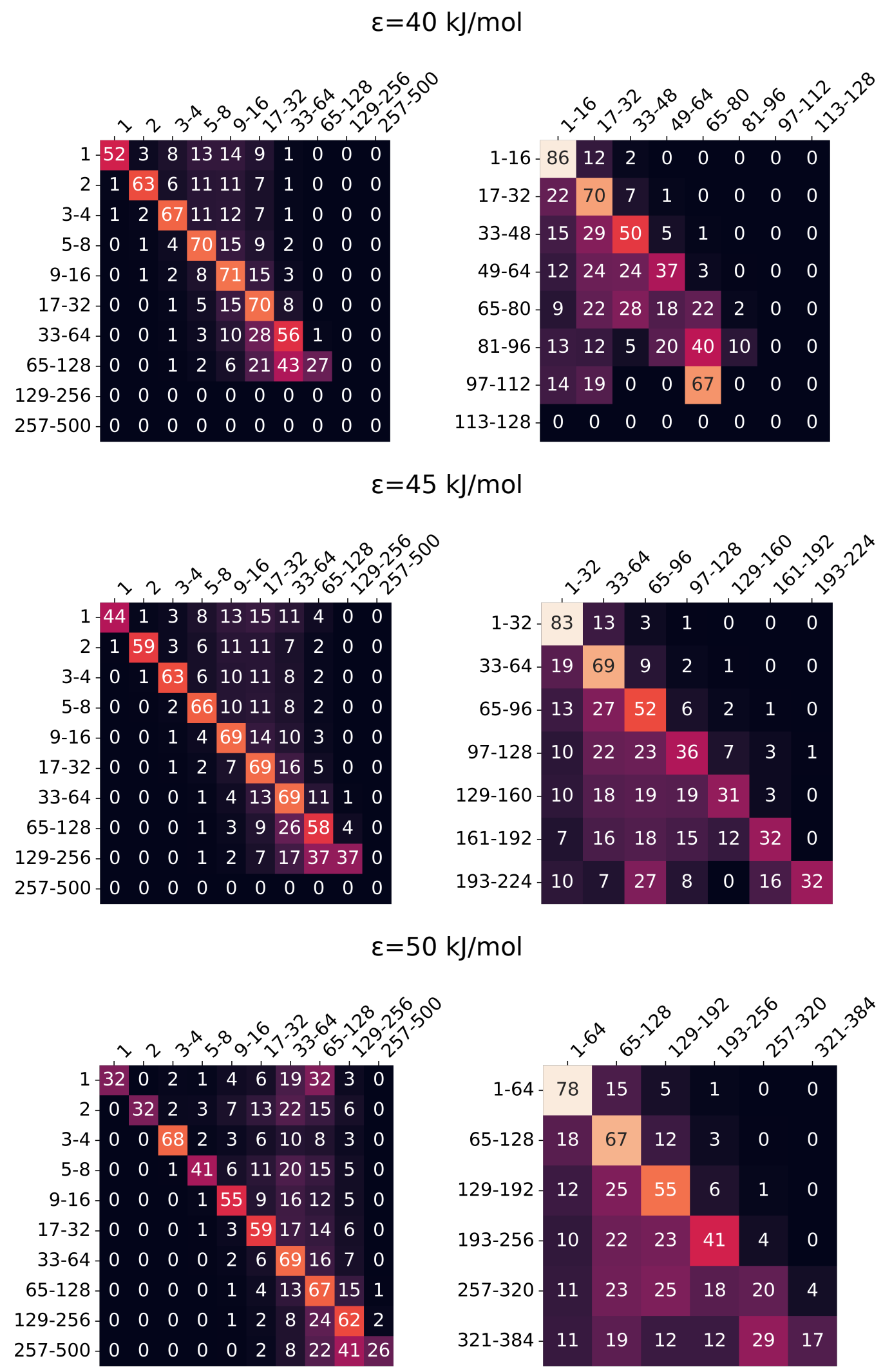

Figure S28. Probability transition matrices for $\mathbf{M}$ systems sampled every $\Delta \tau=3 \mathrm{~ns}$ and cut-off $r_{\text {cut }}=0.6 \mathrm{~nm}$. The data is grouped in size-ranges (binary and regular subdivision in the left and right panels, respectively). 


\section{$\mathrm{T}=340 \mathrm{~K}$}
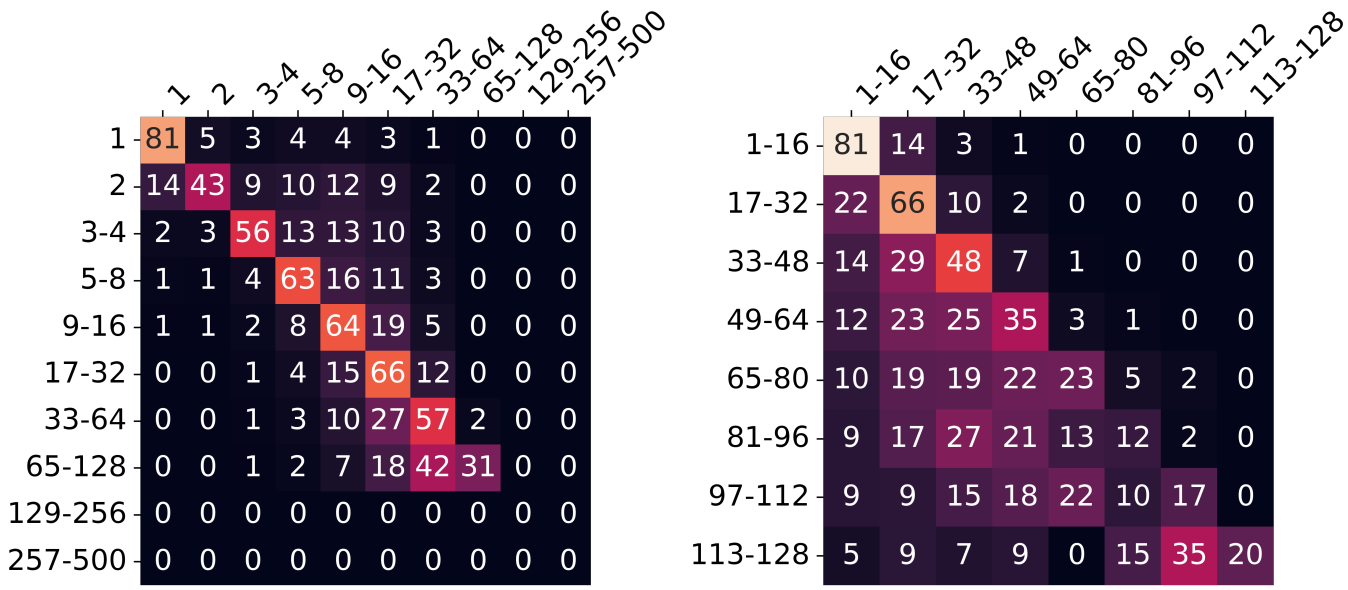

$\mathrm{T}=320 \mathrm{~K}$
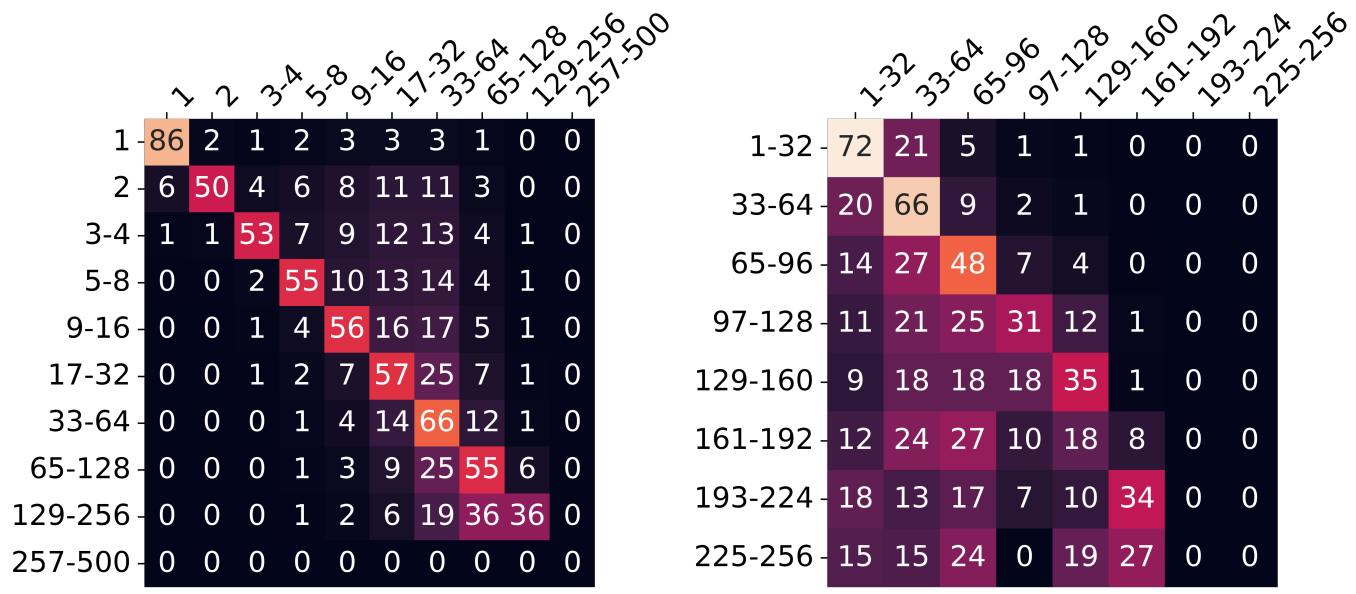

\section{$\mathrm{T}=300 \mathrm{~K}$}

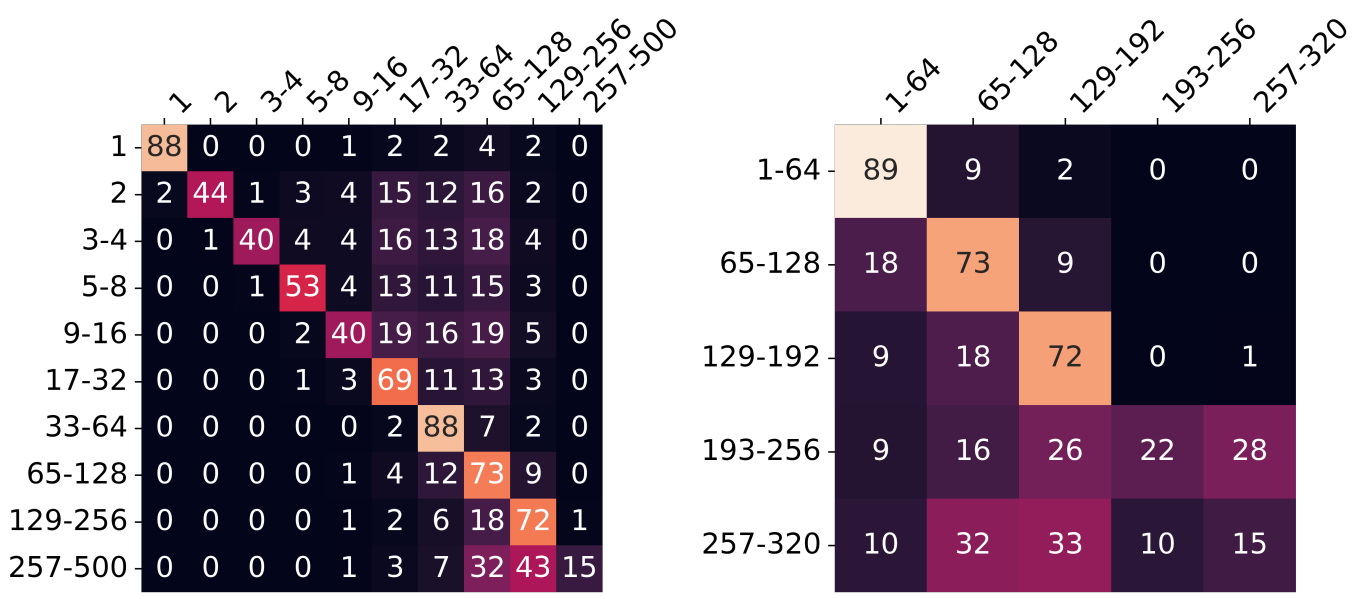

Figure S29. Probability transition matrices for BTA systems sampled every $\Delta \tau=3 \mathrm{~ns}$ and cut-off $r_{\text {cut }}=0.6 \mathrm{~nm}$. The data is grouped in size-ranges (binary and regular subdivision in the left and right panels, respectively). 
HEX \& 40

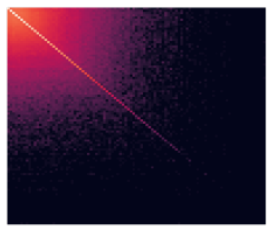

P:

\section{BTA T 340}

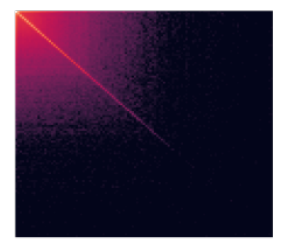

P:

$54 \%$

HEX \& 45

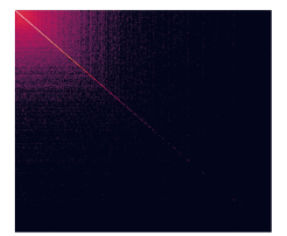

P:

\section{BTA T 320}

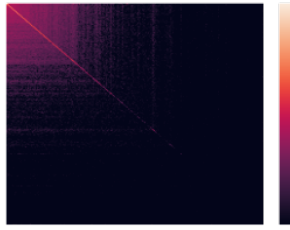

P:

HEX \& 50

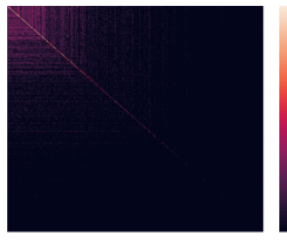

P:

\section{BTA T 300}

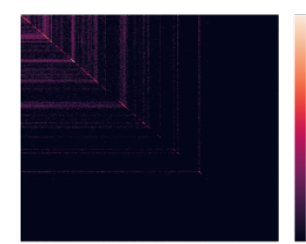

P:

$3 \%$

$18 \%$

$17 \%$

$3 \%$
$A=21 E=4$

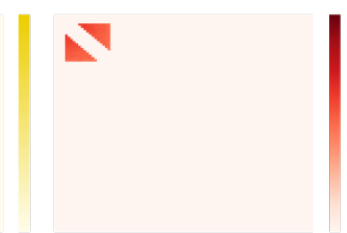

$18 \%$
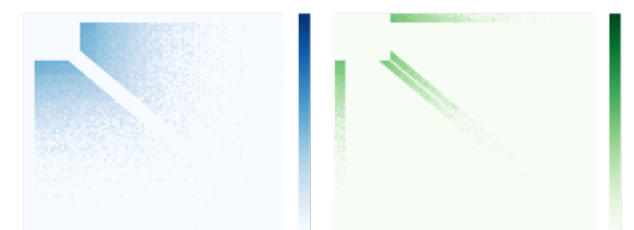

$9 \%$

$21 \%$

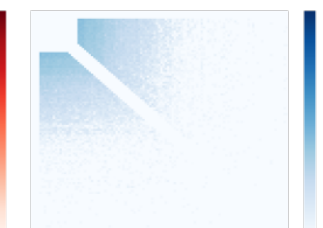

$23 \%$

$10 \%$

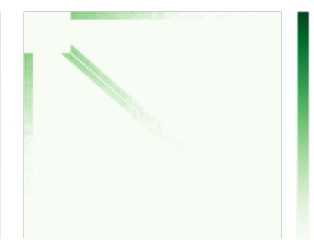

\section{$A=21 E=4$}
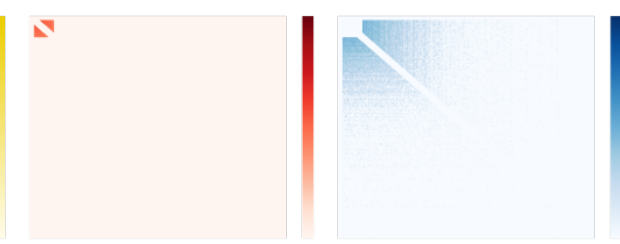

$58 \%$

$14 \%$

$10 \%$
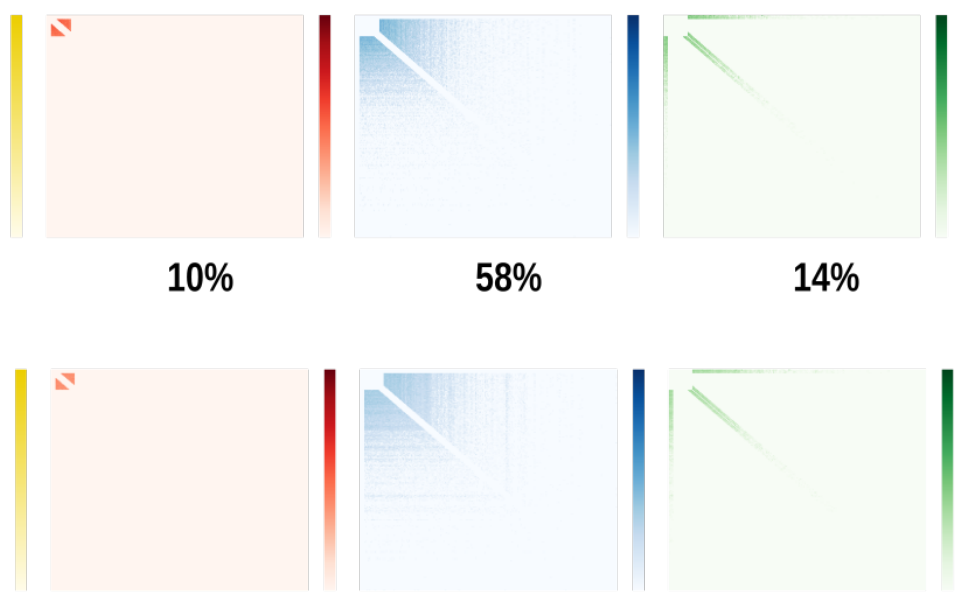

$61 \%$

$15 \%$

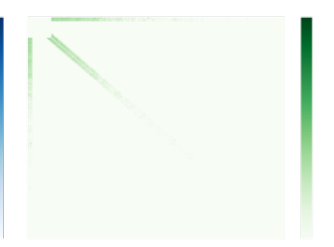

$7 \%$

\section{$A=21 E=4$}

$2 \%$

$83 \%$

$12 \%$
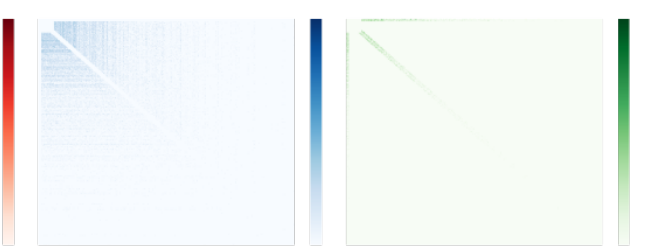


\section{Model Parameters}

We here report the details on the models and force field parameters used for the $\mathbf{M}$ and BTA monomer models, using the GROMACS .itp format for the force field and the GROMACS .gro format for an example configuration.

\section{M monomer force-field}

\begin{tabular}{|c|c|c|c|c|c|}
\hline \multicolumn{6}{|c|}{ [ moleculetype ] } \\
\hline \multicolumn{3}{|c|}{$\begin{array}{l}\text {; molname } \\
\text { MON }\end{array}$} & 1 & & \\
\hline \multicolumn{6}{|c|}{ [ atoms ] } \\
\hline & typ & e res. & r residu & atom cgn & charge \\
\hline+ & M & 1 & MONM & M1 & 0 \\
\hline 2 & P & 1 & MON & P1 & 0 \\
\hline 3 & P & 1 & MON & P2 & 0 \\
\hline 4 & $\mathrm{P}$ & 1 & $\mathrm{MON}$ & P3 & 0 \\
\hline 5 & P & 1 & MON & P 4 & 0 \\
\hline 6 & $\mathrm{P}$ & 1 & MON & P5 & 0 \\
\hline 7 & $\mathrm{P}$ & 1 & MON & P 6 & 0 \\
\hline \multicolumn{6}{|c|}{ [bonds ] } \\
\hline ; & $i$ & j & funct & length & force.c. \\
\hline 1 & 2 & 1 & 0.47 & 20000 & \\
\hline 1 & 3 & 1 & 0.47 & 20000 & \\
\hline 1 & 4 & 1 & 0.47 & 20000 & \\
\hline 1 & 5 & 1 & 0.47 & 20000 & \\
\hline 1 & 6 & 1 & 0.47 & 20000 & \\
\hline 1 & 7 & 1 & 0.47 & 20000 & \\
\hline 2 & 3 & 1 & 0.47 & 20000 & \\
\hline 3 & 4 & 1 & 0.47 & 20000 & \\
\hline 4 & 5 & 1 & 0.47 & 20000 & \\
\hline 5 & 6 & 1 & 0.47 & 20000 & \\
\hline 6 & 7 & 1 & 0.47 & 20000 & \\
\hline 7 & 2 & 1 & 0.47 & 20000 & \\
\hline 2 & 5 & 1 & 0.94 & 15000 & \\
\hline 3 & 6 & 1 & 0.94 & 15000 & \\
\hline & 7 & 1 & 0.94 & 15000 & \\
\hline
\end{tabular}

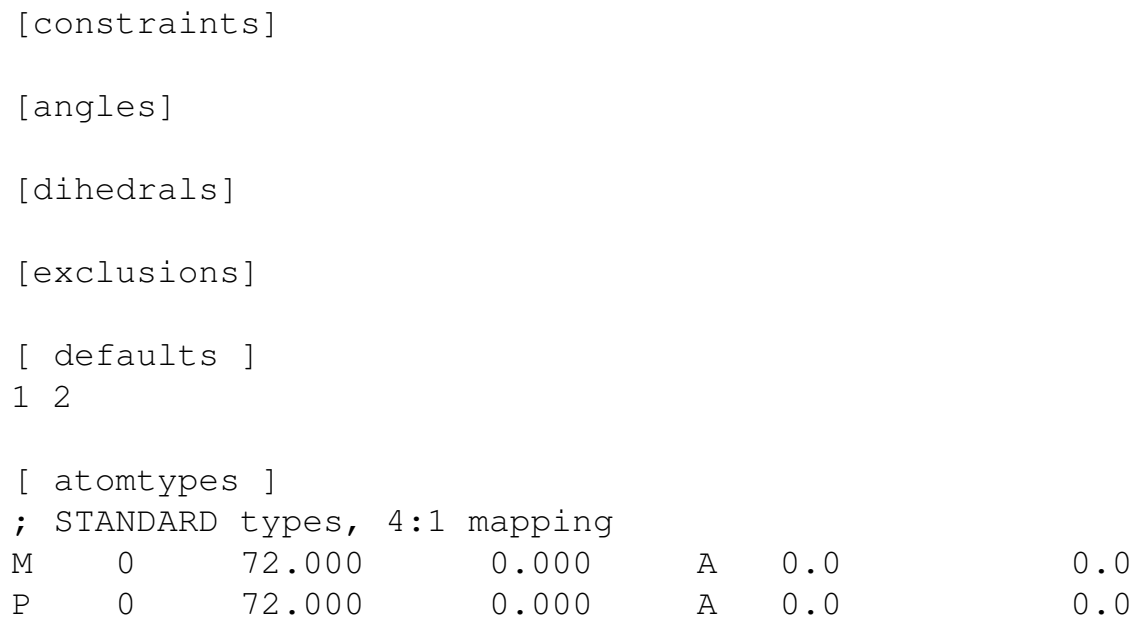




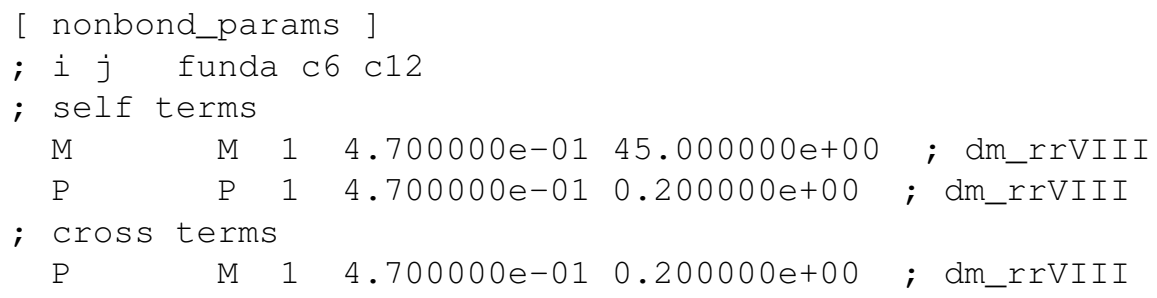

\section{M monomer configuration}

M Monomer

7

$\begin{array}{llrrrr}1 \mathrm{MONM} & \mathrm{M} 1 & 1 & 0.000 & 0.000 & 0.000 \\ 1 \mathrm{MON} & \mathrm{P} 1 & 2 & 0.470 & 0.000 & 0.000 \\ 1 \mathrm{MON} & \mathrm{P} 2 & 3 & 0.235 & 0.407 & 0.000 \\ 1 \mathrm{MON} & \mathrm{P} 3 & 4 & -0.235 & 0.407 & 0.000 \\ 1 \mathrm{MON} & \mathrm{P} 4 & 5 & -0.470 & 0.000 & 0.000 \\ 1 \mathrm{MON} & \mathrm{P} 5 & 6 & -0.235 & -0.407 & 0.000 \\ 1 \mathrm{MON} & \mathrm{P} 6 & 7 & 0.235 & -0.407 & 0.000 \\ 5.98336 & 5.98336 & 5.98336 & & \end{array}$

\section{BTA monomer force-field}

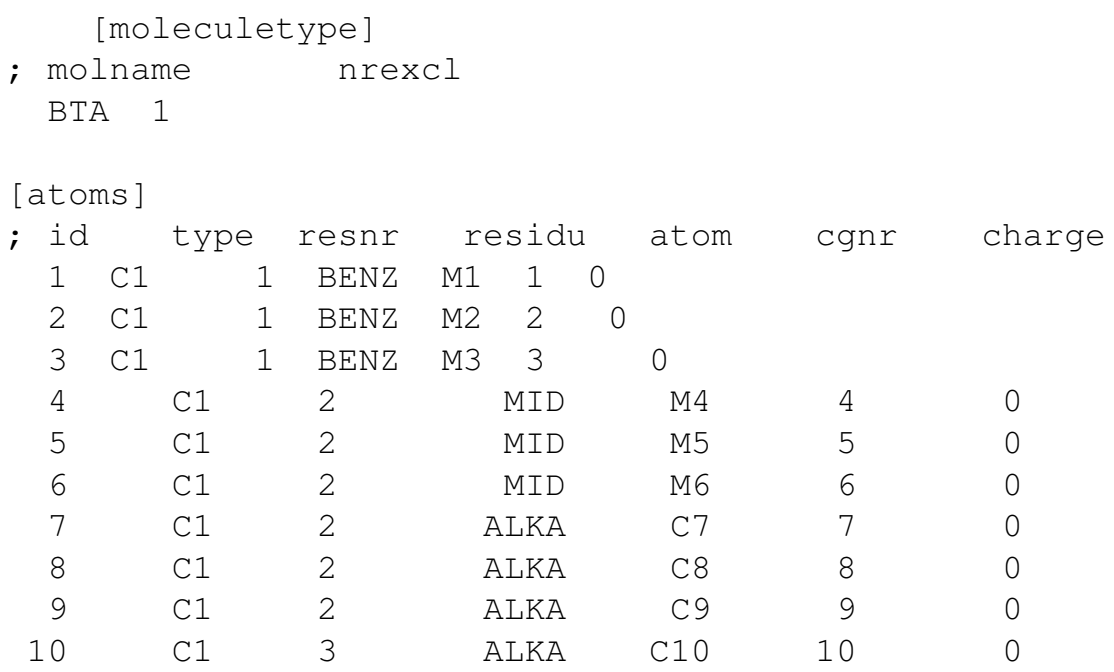




$\begin{array}{rrrrrrr}11 & \text { C1 } & 3 & \text { ALKA } & \text { C11 } & 11 & 0 \\ 12 & \text { C1 } & 3 & \text { ALKA } & \text { C12 } & 12 & 0 \\ 13 & \text { C1 } & 3 & \text { ALKA } & \text { C13 } & 13 & 0 \\ 14 & \text { C1 } & 3 & \text { ALKA } & \text { C14 } & 14 & 0 \\ 15 & \text { C1 } & 3 & \text { ALKA } & \text { C15 } & 15 & 0 \\ 16 & \text { P } 5 & 4 & \text { DIP } & \text { D16 } & 16 & 0 \\ 17 & \text { D } & 5 & \text { HP } & \text { H17 } & 17 & 1.40 \\ 18 & \text { D } & 5 & \text { HN } & \text { H18 } & 18 & -1.40\end{array}$

$\begin{array}{lll}\text { [constraints] } & & \\ 16171 & 0.14 & 22000 \\ 16181 & 0.14 & 22000\end{array}$

[bonds ]

; i j funct length

$\begin{array}{lllll}1 & 2 & 1 & 0.46 & 22000\end{array}$

$\begin{array}{lllll}2 & 3 & 1 & 0.46 & 22000\end{array}$

$\begin{array}{lllll}3 & 1 & 1 & 0.46 & 22000\end{array}$

$\begin{array}{lllll}1 & 16 & 1 & 0.2656 & 22000\end{array}$

$\begin{array}{lllll}2 & 161 & 0.2656 & 22000\end{array}$

$\begin{array}{llll}3 & 161 & 0.2656 & 22000\end{array}$

; BENZ-MID

$\begin{array}{lllll}1 & 4 & 1 & 0.46 & 5500\end{array}$

$\begin{array}{lllll}2 & 5 & 1 & 0.46 & 5500\end{array}$

$\begin{array}{lllll}3 & 6 & 1 & 0.46 & 5500\end{array}$

; MID-MID

$\begin{array}{lllll}4 & 7 & 1 & 0.46 & 5500\end{array}$

$\begin{array}{lllll}5 & 8 & 1 & 0.46 & 5500\end{array}$

$\begin{array}{lllll}6 & 9 & 1 & 0.46 & 5500\end{array}$

; MID-ALKA

$\begin{array}{lllll}7 & 10 & 1 & 0.46 & 5500\end{array}$

$\begin{array}{lllll}8 & 11 & 1 & 0.46 & 5500\end{array}$

$\begin{array}{lllll}9 & 12 & 1 & 0.46 & 5500\end{array}$

; ALKA-ALKA

$\begin{array}{lllll}10 & 13 & 1 & 0.46 & 5500 \\ 11 & 14 & 1 & 0.46 & 5500 \\ 12 & 15 & 1 & 0.46 & 5500\end{array}$

[angles ]

; $i j \mathrm{j}$ funct angle force.c.

$\begin{array}{llllll}3 & 2 & 5 & 2 & 150 & 1500\end{array}$

$\begin{array}{llllll}2 & 1 & 4 & 2 & 150 & 1500\end{array}$

$\begin{array}{llllll}1 & 3 & 6 & 2 & 150 & 1500\end{array}$

;

$\begin{array}{llllll}1 & 2 & 5 & 2 & 150 & 1500 \\ 3 & 1 & 4 & 2 & 150 & 1500 \\ 2 & 3 & 6 & 2 & 150 & 1500\end{array}$

;

$\begin{array}{lllllll}1 & 4 & 7 & 2 & 180.0 & 10\end{array}$

$\begin{array}{lllllll}2 & 5 & 8 & 2 & 180.0 & 10\end{array}$

$\begin{array}{lllllll}3 & 6 & 9 & 2 & 180.0 & 10\end{array}$

;

$\begin{array}{llllll}4 & 7 & 10 & 2 & 180.0 & 10\end{array}$

Page S29 of S31 


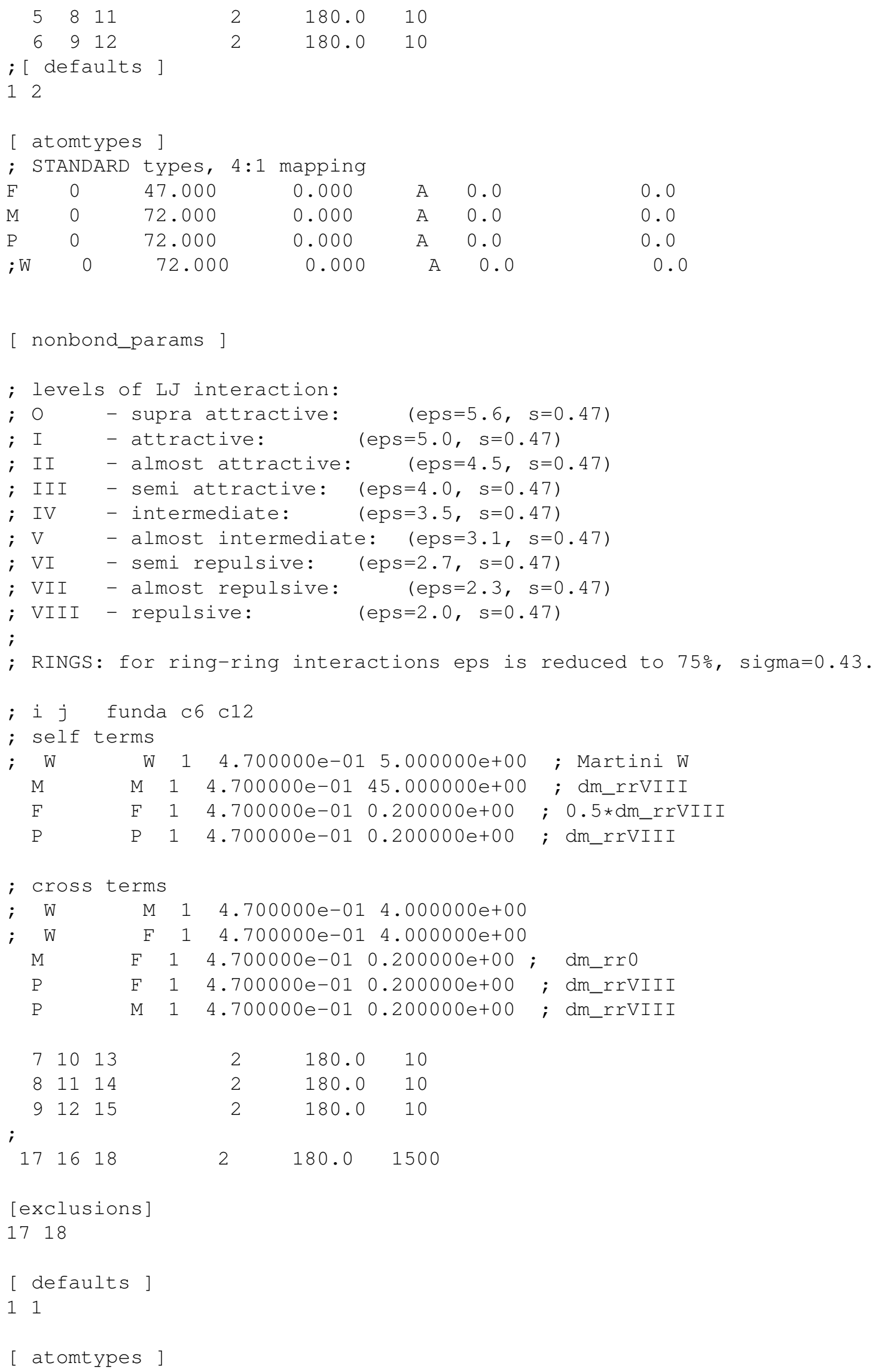




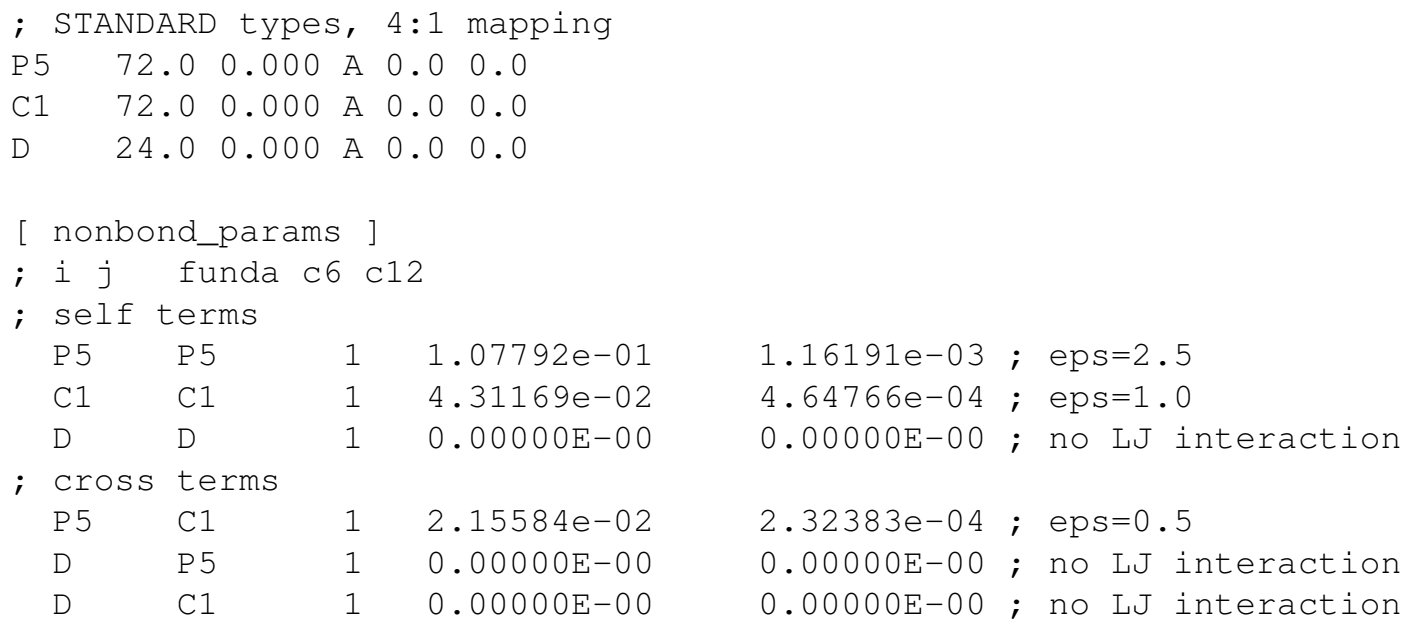

\section{BTA monomer configuration}

BTA

18

$\begin{array}{llrrrr}\text { 1BENZ } & \text { M1 } & 1 & 1.314 & 19.651 & 10.175 \\ \text { 1BENZ } & \text { M2 } & 2 & 1.030 & 19.440 & 10.472 \\ \text { 1BENZ } & \text { M3 } & 3 & 1.215 & 19.879 & 10.543 \\ \text { 2MID } & \text { M4 } & 4 & 1.545 & 19.601 & 9.886 \\ \text { 2MID } & \text { M5 } & 5 & 0.690 & 19.240 & 10.500 \\ \text { 2MID } & \text { M6 } & 6 & 1.397 & 20.158 & 10.819 \\ \text { 3ALKA } & \text { C7 } & 7 & 1.889 & 19.852 & 9.883 \\ \text { 3ALKA } & \text { C8 } & 8 & 0.976 & 19.040 & 10.306 \\ \text { 3ALKA } & \text { C9 } & 9 & 1.345 & 19.875 & 11.031 \\ \text { 4ALKA } & \text { C10 } & 10 & 1.730 & 20.057 & 10.156 \\ \text { 4ALKA } & \text { C11 } & 11 & 1.138 & 19.169 & 10.042 \\ \text { 4ALKA } & \text { C12 } & 12 & 1.078 & 19.631 & 10.893 \\ \text { 4ALKA } & \text { C13 } & 13 & 1.565 & 20.281 & 10.415 \\ \text { 4ALKA } & \text { C14 } & 14 & 1.477 & 19.228 & 9.880 \\ \text { 4ALKA } & \text { C15 } & 15 & 0.769 & 19.336 & 10.813 \\ \text { 5DIP } & \text { D16 } & 16 & 1.173 & 19.674 & 10.385 \\ \text { 6HP } & \text { H17 } & 17 & 1.317 & 19.646 & 10.466 \\ \text { 7HN } & \text { H18 } & 18 & 1.045 & 19.673 & 10.329 \\ \text { 50.00000 } & 50.00000 & 50.00000 & & \end{array}$

